\title{
Reionisation and High-Redshift Galaxies: The View from Quasar Absorption Lines
}

\author{
George D. Becker ${ }^{1,2,5}$, James S. Bolton ${ }^{3}$ and Adam Lidz ${ }^{4}$ \\ ${ }^{1}$ Space Telescope Science Institute, 3700 San Martin Drive, Baltimore, MD 21218, USA \\ ${ }^{2}$ Department of Physics \& Astronomy, University of California, Riverside, 900 University Avenue, Riverside, CA 92521, USA \\ ${ }^{3}$ School of Physics and Astronomy, University of Nottingham, University Park, Nottingham NG7 2RD, UK \\ ${ }^{4}$ Department of Physics and Astronomy, University of Pennsylvania, 209 South 33rd Street, Philadelphia, PA 19104, USA \\ ${ }^{5}$ Email: george.becker@ucr.edu
}

(ReCeIved August 11, 2015; ACCEPTED October 12, 2015)

\begin{abstract}
Determining when and how the first galaxies reionised the intergalactic medium promises to shed light on both the nature of the first objects and the cosmic history of baryons. Towards this goal, quasar absorption lines play a unique role by probing the properties of diffuse gas on galactic and intergalactic scales. In this review, we examine the multiple ways in which absorption lines trace the connection between galaxies and the intergalactic medium near the reionisation epoch. We first describe how the Ly $\alpha$ forest is used to determine the intensity of the ionising ultraviolet background and the global ionising emissivity budget. Critically, these measurements reflect the escaping ionising radiation from all galaxies, including those too faint to detect directly. We then discuss insights from metal absorption lines into reionisation-era galaxies and their surroundings. Current observations suggest a buildup of metals in the circumgalactic environments of galaxies over $z \sim 6$ to 5 , although changes in ionisation will also affect the evolution of metal line properties. A substantial fraction of metal absorbers at these redshifts may trace relatively low-mass galaxies. Finally, we review constraints from the Ly $\alpha$ forest and quasar near zones on the timing of reionisation. Along with other probes of the high-redshift Universe, absorption line data are consistent with a relatively late end to reionisation $(5.5 \lesssim z \lesssim 7)$; however, the constraints are still fairly week. Significant progress is expected to come through improved analysis techniques, increases in the number of known high-redshift quasars from optical and infrared sky surveys, large gains in sensitivity from next-generation observing facilities, and synergies with other probes of the reionisation era.
\end{abstract}

Keywords: dark ages, reionization, first stars - Galaxies: evolution - Galaxies: high-redshift - intergalactic medium quasars: absorption lines

\section{INTRODUCTION}

The period over which the first sources ionised the hydrogen in the intergalactic medium (IGM) and ended the preceding cosmic 'dark ages' is the Epoch of Reionisation (EoR), and determining exactly when and how reionisation happened is a key goal for observational and theoretical cosmology (Loeb \& Furlanetto 2013). Understanding reionisation is important for several reasons. First, it is a major event in our cosmic history that impacted on almost every baryon in the Universe; a better understanding of the EoR is thus vital for developing a complete picture of the Universe's history. Second, detailed measurements of IGM properties during reionisation will strongly inform models of the first luminous sources and high-redshift structure formation, addressing a broad range of open questions regarding the nature of the early Universe. Third, these early generations of ionising sources influenced the formation of subsequent galaxy populations.
Finally, uncertainties in reionisation physics lead to 'nuisance parameters' that may limit our ability to extract cosmological parameters from cosmic microwave background (CMB) and Ly $\alpha$ forest data sets, amongst others.

Although some aspects of reionisation may be understood by studying high-redshift galaxy populations directly, IGM measurements play an important complementary role. After all, it is fundamentally the interplay between the ionising sources and the surrounding intergalactic gas that determines the nature of reionisation; the properties of the intergalactic gas, including its ionisation state, temperature, and metallicity, generally depend on the collective impact of all of the luminous sources while galaxy surveys typically detect only bright sources that lie above survey flux detection limits.

In this context, it has been half a century since Gunn $\&$ Peterson (1965) drew attention to the lack of prominent Ly $\alpha$ absorption troughs in the spectra of the-then newly 


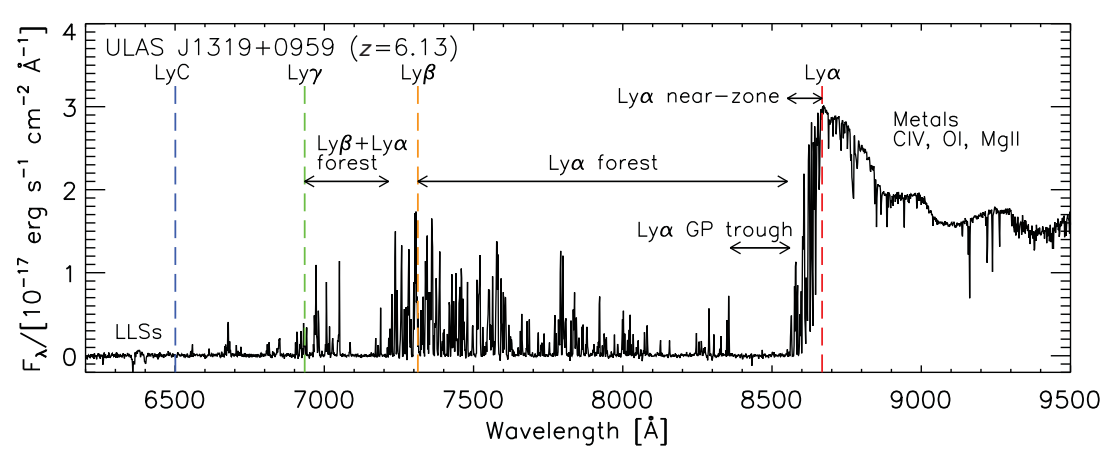

Figure 1. A high signal-to-noise spectrum of the quasar ULAS J1319+0959 at $z=6.13$ from Becker et al. (2015), obtained with the X-Shooter spectrograph on the Very Large Telescope (VLT). The spectrum has been rebinned to $1.5 \AA^{\circ}$ per pixel for presentation purposes. This illustrates many of the features reviewed here-see the text in Section 1 for a description.

discovered-quasars. The absence of strong absorption revealed that there was very little intervening neutral hydrogen in intergalactic space, all the way out to the highestredshift object observed at the time, quasar $3 \mathrm{C} 9$ at $z=2.01$ (Schmidt 1965). In the intervening 50 years, there has been tremendous progress in the study of the IGM using quasar absorption lines, and we now have detailed constraints on many of properties of the IGM and the EoR which extend to the current highest-redshift quasar, ULAS J1120+0641 at $z=7.085$ (Mortlock et al. 2011). The aim of the present article is to review these constraints, examine their implications, and consider the prospects for improving them in the future.

In Section 2, we review the properties of the ultraviolet background (UVB) inferred from the post-reionisation Ly $\alpha$ forest at $z \leq 6$. We compare these measurements to the number of ionising photons expected from star-forming galaxies and quasars, and assess what these data imply for the sources likely responsible for reionising the IGM. In Section 3, we review current observations of IGM metal line abundances at $z>5$, address whether the known galaxy population approaching and during the EoR can account for the observed metal enrichment, and consider the implications of metal line populations for high-redshift galaxy formation. Direct constraints on the reionisation history using quasar absorption line data are then described in Section 4. We also briefly compare these data with other, complementary probes of reionisation. Finally, in Section 5 we conclude with a discussion of future prospects for exploring the EoR with quasar absorption lines.

For further orientation, Figure 1 provides an example of a $z \gtrsim 6$ quasar spectrum in the observed-frame; this illustrates key spectral features used to infer IGM properties approaching the EoR. Redward of the Ly $\alpha$ emission from the quasar (red-dashed line), one can identify a series of metal absorption lines (Section 3). Close to the quasar redshift lies the Ly $\alpha$ proximity or near-zone, where the quasar contributes significantly to ionising the hydrogen in its vicinity (Section 4.5). Next, moving to shorter wavelengths, is the
Ly $\alpha$ absorption forest from intervening neutral hydrogen in the cosmic web (Section 2). This $z \gtrsim 6$ spectrum also shows a complete Gunn-Peterson absorption trough (Section 2.1) above $8400 \AA$ (from hydrogen at $z \gtrsim 5.9$ absorbing in the Ly $\alpha$ line) that continues until the near-zone region. Between the green- and orange-dashed lines, which mark the wavelengths of the Ly $\beta$ and Ly $\gamma$ transitions at the quasar systemic redshift, lies the Ly $\beta$ forest. In this region of the spectrum, high-redshift gas absorbs in the Ly $\beta$ line and at lower redshift, foreground gas absorbs in $\operatorname{Ly} \alpha$ (Section 4.3). At even shorter wavelengths, overlapping higher-order Lyman series transitions occur. Finally, below the line marked ' $\mathrm{LyC}$ ' there is continuum absorption from neutral hydrogen: photons at these wavelengths - with rest frame wavelength $\lambda \leq 912 \AA$ - are energetic enough to photoionise hydrogen atoms. In lower-redshift quasar spectra where there is less overall absorption, Lyman-limit systems (LLSs) — absorbers that have an optical depth of unity to photons at the hydrogen photoionisation edge - can be identified here. LLSs, along with cumulative absorption from lower-column density absorbers, set the mean free path to ionising photons in the IGM (Section 2.4).

\section{THE UV BACKGROUND}

The UVB is a key probe of the sources of hydrogen ionising photons $(E \geq 13.6 \mathrm{eV})$ in the post-reionisation era at $z<6$; its intensity and spectral shape provides a complete census of ionising photon production and its evolution with redshift (Haardt \& Madau 1996, 2012; Faucher-Gigu et al. 2009). One of the primary observational techniques used to probe the UVB is quasar absorption line spectroscopy. The Ly $\alpha$ forest-the observable manifestation of the intergalactic neutral hydrogen that traces the cosmic web of large-scale structure (see e.g. Rauch 1998; Meiksin 2009)—is particularly important in this regard. In this section, we discuss the theoretical and observational framework on which UVB measurements using the Ly $\alpha$ forest are based, and examine the 
implications of these data for the properties of high-redshift galaxies during the reionisation era.

\subsection{The Gunn-Peterson effect}

It is instructive to first review the relationship between the neutral hydrogen fraction of the IGM and the intergalactic Ly $\alpha$ opacity. Gunn \& Peterson (1965) demonstrated that quasar spectra are a useful probe of the intergalactic neutral hydrogen density. If the intervening IGM along a quasar line of sight contains sufficient neutral hydrogen, the transmitted flux blueward of a quasar's Ly $\alpha$ emission line (at rest-frame wavelength $\lambda_{\alpha}=1215.67^{\circ} \mathrm{A}$ ) will be completely attenuated, producing a saturated absorption trough.

The Gunn \& Peterson (1965) argument is as follows. Consider light emitted by a quasar at redshift $z_{\mathrm{q}}$ passing through a uniform IGM with proper neutral hydrogen number density $n_{\mathrm{HI}}(z)$. This light is observed at redshift $z<z_{\mathrm{q}}$ with frequency $v=v_{\alpha} /(1+z)$, such that the emitted photons have redshifted into Ly $\alpha$ resonance with the local IGM. The total optical depth along the line of sight is then

$$
\tau_{\mathrm{GP}}^{\alpha}=\int_{0}^{z_{\mathrm{q}}} \sigma_{\mathrm{s}}[v(1+z)] n_{\mathrm{HI}}(z) \frac{\mathrm{d} l}{\mathrm{~d} z} \mathrm{~d} z
$$

where $\mathrm{d} l / \mathrm{d} z=-c /[H(z)(1+z)]$ is the proper line element. Ignoring line-broadening effects, the effective scattering cross-section, $\sigma_{\mathrm{s}}$, may be approximated by a Dirac delta function peaked at $v_{\alpha}$

$$
\sigma_{\mathrm{s}}[v(1+z)]=\sigma_{\alpha} v_{\alpha} \delta\left[v(1+z)-v_{\alpha}\right],
$$

where $\sigma_{\alpha}=4.48 \times 10^{-18} \mathrm{~cm}^{2}$ is the Ly $\alpha$ cross-section. Changing the variable of integration to $v$, where $\mathrm{d} l / \mathrm{d} v=$ $\lambda_{\alpha}(1+z) / H(z)$ and noting that $\tau_{\mathrm{GP}}^{\alpha}=0$ when $v \leq v_{\alpha} /(1+$ $z)$ then yields

$$
\tau_{\mathrm{GP}}^{\alpha} \simeq \frac{\sigma_{\alpha} c n_{\mathrm{HI}}(z)}{H_{0} \Omega_{\mathrm{m}}^{1 / 2}(1+z)^{3 / 2}},
$$

using the high-redshift $(z \geq 2)$ approximation for the Hubble parameter, $H(z) \simeq H_{0} \Omega_{\mathrm{m}}^{1 / 2}(1+z)^{3 / 2}$. Identifying $\left\langle x_{\mathrm{HI}}\right\rangle=$ $n_{\mathrm{HI}} / \bar{n}_{\mathrm{H}}$ as the average neutral hydrogen fraction, the GunnPeterson optical depth at the background density, $\bar{n}_{\mathrm{H}}=$ $\rho_{\text {crit }} \Omega_{\mathrm{b}}(1-Y)(1+z)^{3} / m_{\mathrm{H}}$, is then

$$
\begin{aligned}
\tau_{\mathrm{GP}}^{\alpha} \simeq 2.3 \times & 10^{5}\left\langle x_{\mathrm{HI}}\right\rangle\left(\frac{\Omega_{\mathrm{b}} h^{2}}{0.022}\right)\left(\frac{\Omega_{\mathrm{m}} h^{2}}{0.142}\right)^{-1 / 2} \\
& \times\left(\frac{1-Y}{0.76}\right)\left(\frac{1+z}{5}\right)^{3 / 2},
\end{aligned}
$$

where $Y$ is the primordial helium fraction by mass.

The transmittance shortward of a quasar's Ly $\alpha$ emission line is just $\mathrm{e}^{-\tau_{\mathrm{GP}}^{\alpha}}$. Consequently, even for a modest neutral fraction of $\left\langle x_{\mathrm{HI}}\right\rangle \sim 10^{-4.5}$ the Gunn-Peterson optical depth is fully saturated (i.e. $\mathrm{e}^{-\tau_{\mathrm{GP}}^{\alpha}} \approx 0$ ). Observationally, the decline in the observed Ly $\alpha$ opacity and especially the absence of Ly $\alpha$ troughs in quasar spectra at $z<5.5$ indicates the volume-weighted neutral hydrogen fraction in the IGM is very small by this redshift (Becker et al. 2001; Djorgovski et al. 2001; Songaila 2004; Fan et al. 2006, see also McGreer et al. 2015)—we shall return to this point in Section 4.2.

\subsection{The $L y \alpha$ forest opacity and the metagalactic hydrogen ionisation rate}

The Gunn \& Peterson (1965) argument implies the IGM is highly ionised along observed quasar sight-lines at $z<5.5$. However, it does not directly relate the observed Ly $\alpha$ opacity to the quantity of interest here - the intensity of the UVB. To progress further, we must recognise that intergalactic Ly $\alpha$ absorption arises not from a uniform medium, but the continuous, fluctuating distribution of baryons which forms through hierarchical structure formation within cold dark matter models. Estimates of the ionising photon production by sources in the early Universe rely on the resultant relationship between the opacity of the Ly $\alpha$ 'forest' of absorption lines and the intensity of the UVB.

We may consider the relationship between the Ly $\alpha$ forest opacity and the UVB as follows. The UVB-specific intensity at redshift $z_{0}$ and frequency $v_{0}$, is given by (e.g. Haardt \& Madau 1996; Faucher-Gigu et al. 2008b; Becker \& Bolton 2013)

$$
J\left(v_{0}, z_{0}\right)=\frac{1}{4 \pi} \int_{z_{0}}^{\infty} \mathrm{d} z \frac{\mathrm{d} l}{\mathrm{~d} z} \frac{\left(1+z_{0}\right)^{3}}{(1+z)^{3}} \epsilon(\nu, z) \mathrm{e}^{-\bar{\tau}\left(v_{0}, z_{0}, z\right)} .
$$

This expression is obtained by solving the cosmological radiative transfer equation, where $\epsilon(\nu, z)$ is the proper specific emissivity, $v=v_{0}(1+z) /\left(1+z_{0}\right)$ and $\bar{\tau}\left(v_{0}, z_{0}, z\right)$ is the intervening effective optical depth for photons with frequency $v_{0}$ at redshift $z_{0}$ that were emitted at redshift $z$ (see Equation (10) later). The number of hydrogen atoms photoionised per unit time, $\Gamma_{\mathrm{HI}}$, is then related to the specific intensity of the UVB by

$$
\Gamma_{\mathrm{HI}}(z)=\int_{v_{\mathrm{LL}}}^{\infty} \frac{4 \pi J(v, z)}{h_{\mathrm{P}} v} \sigma_{\mathrm{HI}}(v) \mathrm{d} v \simeq \frac{4 \pi J_{\mathrm{LL}} \sigma_{\mathrm{LL}}}{h_{\mathrm{P}}\left(\alpha_{\mathrm{bg}}+3\right)},
$$

where $\sigma_{\mathrm{HI}}$ is the $\mathrm{H}$ Iphotoionisation cross-section (see e.g. Verner et al. 1996), $h_{\mathrm{P}}$ is Planck's constant and $v_{\mathrm{LL}}$ is the photon frequency at the Lyman limit. The final step makes the simplifying assumption of a power-law UVB spectrum, $J(v)=J_{\mathrm{LL}}\left(\nu / v_{\mathrm{LL}}\right)^{-\alpha_{\mathrm{bg}}}$, and approximates ${ }^{1}$ the photoionisation cross-section as $\sigma_{\mathrm{HI}} \simeq \sigma_{\mathrm{LL}}\left(\nu / \nu_{\mathrm{LL}}\right)^{-3}$, where $\sigma_{\mathrm{LL}}=6.35 \times 10^{-18} \mathrm{~cm}^{2}$.

The photoionisation rate can be related to the neutral hydrogen fraction in the IGM. Following reionisation, hydrogen in the low-density IGM is in ionisation equilibrium with the UVB. If ignoring collisional ionisations (appropriate for the low temperatures, $T<10^{5} \mathrm{~K}$ associated with gas in the Ly $\alpha$ forest),

$$
n_{\mathrm{HI}} \Gamma_{\mathrm{HI}}=n_{\mathrm{e}} n_{\mathrm{HII}} \alpha_{\mathrm{HII}}(T)
$$

\footnotetext{
${ }^{1}$ A scaling of $\sigma_{\mathrm{HI}} \propto v^{-2.75}$ is a better fit to the true frequency dependence at energies close to 1 Ryd (e.g. Osterbrock \& Ferland 2006); however, we adopt the commonly used approximation $\sigma_{\mathrm{HI}} \propto v^{-3}$ for simplicity.
} 
Here, $n_{\mathrm{HI}}, n_{\mathrm{HI}}$, and $n_{\mathrm{e}}$ are the number densities of neutral hydrogen, ionised hydrogen, and free electrons, and $\alpha_{\mathrm{HII}}(T)$ is the temperature-dependent radiative recombination coefficient. We adopt the case-A recombination rate $\alpha_{\mathrm{HI}}(T)=$ $4.063 \times 10^{-13}\left(T / 10^{4} \mathrm{~K}\right)^{-0.72} \mathrm{~cm}^{3} \mathrm{~s}^{-1}$ in what follows, although more accurate fits are available (e.g. Verner \& Ferland 1996). If the hydrogen is highly ionised, then $n_{\mathrm{HII}} \simeq n_{\mathrm{H}}$, $n_{\mathrm{e}} \simeq n_{\mathrm{H}}\left(1+n_{\mathrm{He}} / n_{\mathrm{H}}\right)$, and Equation (6) may be rewritten as

$$
\begin{gathered}
x_{\mathrm{HI}} \simeq 9.6 \times 10^{-6} \Delta \frac{\left(1+x_{\mathrm{H}}\right)}{\Gamma_{-12}}\left(\frac{T}{10^{4} \mathrm{~K}}\right)^{-0.72}\left(\frac{\Omega_{\mathrm{b}} h^{2}}{0.022}\right) \\
\times\left(\frac{1-Y}{0.76}\right)\left(\frac{1+z}{5}\right)^{3},
\end{gathered}
$$

where $x_{\mathrm{HI}}=n_{\mathrm{HI}} / n_{\mathrm{H}}$ is the $\mathrm{H}$ Ifraction, $\Delta=\rho / \bar{\rho}$ is the fractional overdensity, $\chi_{\mathrm{He}}=\frac{\eta Y}{4(1-Y)}$ accounts for electrons released by singly $(\eta=1)$ and doubly $(\eta=2)$ ionised helium ${ }^{2}$, and $\Gamma_{-12}=\Gamma_{\mathrm{HI}} / 10^{-12} \mathrm{~s}^{-1}$.

Finally, note that photoionisation by the UVB also heats the low-density IGM (Miralda-Escudé \& Rees 1994). Well after reionisation, photoheating and cooling due to the adiabatic expansion of the Universe produces a well-defined temperature-density relation ${ }^{3}$ for gas overdensities $\Delta \lesssim 10$, where $T=T_{0} \Delta^{\gamma-1}$ (Hui \& Gnedin 1997; McQuinn \& Upton Sanderbeck 2015). Typically, the temperature at mean density is $10^{3.7} \leq T_{0} \leq 10^{4.3} \mathrm{~K}$, while the slope of the relation is $1 \leq \gamma \leq 1.6$ (Schaye et al. 2000; Ricotti, Gnedin, \& Shull 2000; McDonald et al. 2001; Lidz et al. 2010; Becker et al. 2011a; Garzilli et al. 2012; Rudie, Steidel, \& Pettini 2012; Bolton et al. 2014; Boera et al. 2014). Combining this temperature-density relation with Equations (2) and (6) gives

$$
\begin{aligned}
\tau_{\mathrm{FGPA}}^{\alpha} \simeq & 2.2 \Delta^{\beta} \frac{\left(1+\chi_{\mathrm{He}}\right)}{\Gamma_{-12}}\left(\frac{T_{0}}{10^{4} \mathrm{~K}}\right)^{-0.72}\left(\frac{\Omega_{\mathrm{b}} h^{2}}{0.022}\right)^{2} \\
& \times\left(\frac{\Omega_{\mathrm{m}} h^{2}}{0.141}\right)^{-1 / 2}\left(\frac{1-Y}{0.76}\right)^{2}\left(\frac{1+z}{5}\right)^{9 / 2},
\end{aligned}
$$

where $\beta=2-0.72(\gamma-1)$. This is the Fluctuating GunnPeterson Approximation (FGPA, e.g. Rauch et al. 1997; Weinberg et al. 1999). It neglects redshift space distortions and spatial fluctuations in the UVB, but elucidates the dependence of the Ly $\alpha$ forest optical depth on underlying physical quantities. Importantly, this includes the photoionisation rate $\Gamma_{\mathrm{HI}}$ (and hence specific intensity $J(v, z)$ ) of the UVB.

\footnotetext{
${ }^{2}$ The reionisation of neutral helium, with an ionisation potential of $24.6 \mathrm{eV}$, is thought to occur at the same time as $\mathrm{H}$ Ireionisation (Friedrich et al. 2012). In contrast, the reionisation of singly ionised helium, with an ionisation potential $54.4 \mathrm{eV}$, is not expected to complete until $z \simeq 2-3$ due to the relative scarcity of ionising sources (most likely active galactic nuclei, AGN) with hard spectra at higher redshifts (Furlanetto \& Oh 2008; McQuinn et al. 2009; Compostella, Cantalupo, \& Porciani 2014).

${ }^{3}$ Immediately following (inhomogeneous) hydrogen reionisation, the temperature-density relation is instead expected to exhibit considerable scatter (Bolton, Meiksin, \& White 2004; Furlanetto \& Oh 2009; Lidz \& Malloy 2014), and may even be inverted, $\gamma<1$ (Trac, Cen \& Loeb 2008). Evidence for fluctuations in the thermal state of the IGM at high redshift may thus provide further, indirect evidence of the end stages of reionisation.
}

\subsection{Measurements of the photoionisation rate}

With the relationship between the Ly $\alpha$ forest opacity and the UVB intensity established, we now consider how measurements are made in practice. There are two main techniques employed in the literature to measure the metagalactic photoionisation rate, $\Gamma_{\mathrm{HI}}$ : modelling the mean transmitted flux in the Ly $\alpha$ forest (Rauch et al. 1997) and the quasar proximity effect (Murdoch et al. 1986; Bajtlik, Duncan, \& Ostriker 1988).

The first approach typically relies on using numerical simulations of structure formation to reproduce the observed mean transmission in the Ly $\alpha$ forest, $\langle F\rangle=\left\langle\mathrm{e}^{-\tau_{\alpha}}\right\rangle$. Mock Ly $\alpha$ forest spectra are extracted from simulations performed using a spatially uniform, time-dependent UVB model (e.g. Haardt \& Madau 1996, 2012; Faucher-Gigu et al. 2009). The amplitude of the UVB model is then treated as an adjustable parameter; since in photoionisation equilibrium $\tau_{\alpha} \propto \Gamma_{\mathrm{HI}}^{-1}$ (e.g. Equation (8)), rescaling the simulated Ly $\alpha$ optical depths is straightforward. The photoionisation rate $\Gamma_{\mathrm{HI}}$ (and hence $J(v)$, if the UVB spectral shape is known) may then be obtained by matching the mean transmission in the simulated spectra to observational measurements at $2<z<6$ (e.g. Kim et al. 2002; Schaye et al. 2003; Kirkman et al. 2005; Fan et al. 2006; Faucher-Giguère et al. 2008a; Pâris et al. 2011; Becker et al. 2013, 2015).

This method has been widely applied to Ly $\alpha$ forest data at $z>2$ (McDonald \& Miralda-Escudé 2001; Meiksin \& White 2003; Tytler et al. 2004; Bolton et al. 2005; Faucher-Gigu et al. 2008b; Wyithe \& Bolton 2011; Rollinde et al. 2013; Becker \& Bolton 2013). However, this approach will start to break down approaching $z=6$, when the assumption of a spatially uniform UVB no longer holds (see also Section 2.7). Corrections for the effect of spatial fluctuations in the UVB are therefore applied to the measurements (Meiksin \& White 2003; Bolton \& Haehnelt 2007c). The mean transmitted flux furthermore depends on the IGM temperature and cosmology (e.g. Equation (8)). Independent constraints on these quantities are therefore required, and the historical range of estimates for $\Gamma_{\mathrm{HI}}$ in literature are in part attributable to differing assumptions for these parameters.

The second widely used approach, the line-of-sight proximity effect, estimates $\Gamma_{\mathrm{HI}}$ by quantifying the reduction in the average Ly $\alpha$ opacity approaching the systemic redshift of a quasar (Weymann, Carswell, \& Smith 1981; Carswell et al. 1982). The ionising radiation from a quasar will locally ionise the hydrogen in the IGM to a higher degree than the UVB. Ignoring peculiar velocities and spatial variations in gas temperature, since $\tau_{\alpha} \propto \Gamma_{\mathrm{HI}}^{-1}$ it follows that the Ly $\alpha$ optical depth as a function of proper distance $r$ from a quasar is

$$
\tau_{\alpha}^{\mathrm{UVB}+\mathrm{Q}}=\frac{\tau_{\alpha}^{\mathrm{UVB}}}{1+\Gamma_{\mathrm{HI}}^{\mathrm{Q}}(r) / \Gamma_{\mathrm{HI}}^{\mathrm{UVB}}},
$$

where $\tau_{\alpha}^{\mathrm{UVB}}$ is the typical optical depth in the Ly $\alpha$ forest, $\Gamma_{\mathrm{HI}}^{\mathrm{Q}}(r)$ is the ionisation rate of the quasar, and $\Gamma_{\mathrm{HI}}^{\mathrm{UVB}}$ is the 


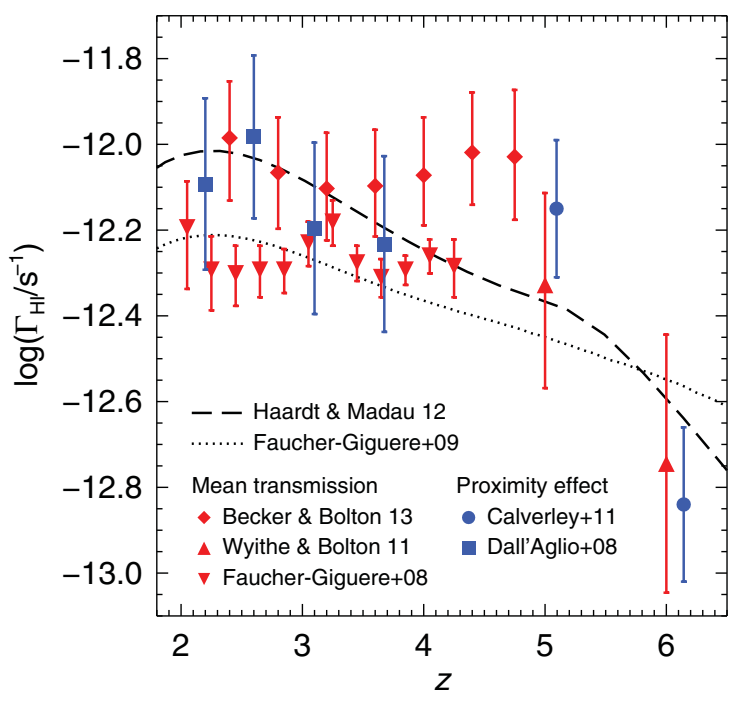

Figure 2. Summary of recent $\Gamma_{\mathrm{HI}}$ measurements obtained from the mean Ly $\alpha$ forest transmission (Faucher-Gigu et al. 2008b; Wyithe \& Bolton 2011; Becker \& Bolton 2013) and the proximity effect (Dall'Aglio, Wisotzki, \& Worseck 2008; Calverley et al. 2011). Note that some of the data points have been offset by $\Delta z=0.05$ for clarity, and the Dall'Aglio et al. (2008) data have been rebinned and converted from a specific intensity assuming $\alpha_{\mathrm{bg}}=1.5$. These data are furthermore quoted directly from the literature. However, caution must be exercised with any direct comparison here as differing assumptions for systematic uncertainties in these studies such as e.g. the IGM temperature, can increase the scatter in the measurements. For further discussion of this point, see Becker \& Bolton (2013). For comparison, the dashed and dotted curves display the empirically calibrated UVB models constructed by Haardt \& Madau (2012) and Faucher-Gigu et al. (2009), respectively. These are based on the expected contribution to the UVB from star-forming galaxies and quasars, and an empirical model for the ionising opacity of the IGM.

UVB ionisation rate. If the absolute magnitude, redshift and spectral energy distribution of the quasar are known, $\Gamma_{\mathrm{HI}}^{\mathrm{Q}}(r)$ may be computed with Equation (5). Estimates for $\tau_{\alpha}^{\mathrm{UVB}+\mathrm{Q}}$ and $\tau_{\alpha}^{\mathrm{UVB}}$ then lead to $\Gamma_{\mathrm{HI}}^{\mathrm{UVB}}$.

The optical depth, however, is not a directly observable quantity; early proximity effect analyses therefore focussed on measuring the number density of $\operatorname{Ly} \alpha$ absorption lines blueward of a quasar's Ly $\alpha$ emission line (e.g. Murdoch et al. 1986; Bajtlik et al. 1988; Giallongo et al. 1996; Scott et al. 2000). More recent approaches have instead analysed the transmitted flux, $F=\mathrm{e}^{-\tau}$, often combined with numerical simulations of the IGM which model the density, temperature, and velocity field around quasar host haloes in detail (Faucher-Giguère et al. 2008a; Dall'Aglio et al. 2008; Calverley et al. 2011). In addition to modelling the atypical environment of quasars, care must be taken to avoid further potential biases in the measurements arising from line-ofsight variations in the IGM density distribution (e.g Loeb \& Eisenstein 1995; Rollinde et al. 2005; Guimarães et al. 2007; Partl et al. 2011).

The typical values obtained using both these techniques are $\Gamma_{\mathrm{HI}} \sim 10^{-12} \mathrm{~s}^{-1}$ at $2 \leq z \leq 4$, declining by approximately a factor of 2 and 4 approaching reionisation at $z=5$ and $z=6$, respectively. Some recent measurements are summarised in Figure 2. Inferences about the underlying ionising source population can be made by comparing these measurements to estimates of the emissivity from known ionising sources at $2<z<6$, obtained by integrating observed quasar and Lyman break galaxy (LBG) luminosity functions. We discuss this comparison in more detail in Section 2.6. However, this first requires converting $\Gamma_{\mathrm{HI}}$ into an emissivity, which itself relies on estimates for the typical mean free path for ionising photons and its evolution with redshift.

\subsection{The mean free path at the Lyman limit}

Consider the mean free path for ionising photons in an IGM populated by Poisson distributed H Iabsorbers, with column densities $N_{\mathrm{HI}}$ described by the column density distribution function (CDDF), $f\left(N_{\mathrm{HI}}, z\right)=\partial^{2} n / \partial N_{\mathrm{HI}} \partial z$. The intervening effective optical depth (see also Equation (4)) for photons with frequency $v_{0}$ at redshift $z_{0}$ that were emitted at redshift $z$ is then (Paresce, McKee, \& Bowyer 1980)

$$
\bar{\tau}\left(v_{0}, z_{0}, z\right)=\int_{z_{0}}^{z} \mathrm{~d} z^{\prime} \int_{0}^{\infty} \mathrm{d} N_{\mathrm{HI}} f\left(N_{\mathrm{HI}}, z^{\prime}\right)\left(1-\mathrm{e}^{-\tau_{\nu}}\right),
$$

where $\tau_{v}=\sigma_{\mathrm{HI}}(v) N_{\mathrm{HI}}$. Parameterising the CDDF as $f\left(N_{\mathrm{HI}}, z\right)=N_{0} N_{\mathrm{HI}}^{-\beta_{\mathrm{N}}}(1+z)^{\beta_{z}}$ and evaluating the integral yields (e.g. Faucher-Gigu et al. 2008b)

$$
\begin{gathered}
\bar{\tau} \quad=N_{0} \frac{\Gamma\left(2-\beta_{\mathrm{N}}\right)\left(1+z_{0}\right)^{3\left(\beta_{\mathrm{N}}-1\right)}}{\left(\beta_{\mathrm{N}}-1\right)\left(\beta_{\mathrm{z}}-3 \beta_{\mathrm{N}}+4\right)}\left(\frac{v_{0}}{v_{\mathrm{LL}}}\right)^{-3\left(\beta_{\mathrm{N}}-1\right)} \\
\times \sigma_{\mathrm{LL}}^{\beta_{\mathrm{N}}-1}\left[(1+z)^{\beta_{\mathrm{z}}-3 \beta_{\mathrm{N}}+4}-\left(1+z_{0}\right)^{\beta_{\mathrm{z}}-3 \beta_{\mathrm{N}}+4}\right],
\end{gathered}
$$

where $\Gamma$ is the Gamma function. The mean free path is then the distance a photon can travel before encountering an optical depth of unity. Noting that $\mathrm{d} l / \mathrm{d} \bar{\tau}=(\mathrm{d} l / \mathrm{d} z) /(\mathrm{d} \bar{\tau} / \mathrm{d} z) \simeq$ $\lambda_{\text {mfp }}$ when $\Delta \bar{\tau}=1$ thus leads to

$$
\begin{aligned}
\lambda_{\mathrm{mfp}}(\nu) \simeq \frac{c\left(\beta_{\mathrm{N}}-1\right)}{N_{0} \beta_{\mathrm{LL}}^{-1} \Gamma\left(2-\beta_{\mathrm{N}}\right)}\left(\frac{v}{v_{\mathrm{LL}}}\right)^{3\left(\beta_{\mathrm{N}}-1\right)} \\
\times \frac{1}{H_{0} \Omega_{\mathrm{m}}^{1 / 2}(1+z)^{\beta^{2}}+5 / 2}
\end{aligned}
$$

which gives an analytical approximation for the mean free path for ionising photons at $z \gtrsim 2$. This may be written more compactly as $\lambda_{\text {mfp }}=\lambda_{\mathrm{LL}}(z)\left(v / v_{\mathrm{LL}}\right)^{3\left(\beta_{\mathrm{N}}-1\right)}$, where $\lambda_{\mathrm{LL}}(z)$ is the mean free path at the Lyman limit. In general, therefore, higher frequency photons have a larger mean free path and $\lambda_{\mathrm{LL}}(z)$ decreases with increasing redshift, but the precise normalisation, frequency, and redshift dependence of Equation (11) relies on an accurate observational determination of the CDDF or a related quantity.

Many surveys have attempted to infer $\lambda_{\mathrm{LL}}(z)$ by measuring $f\left(N_{\mathrm{HI}}, z\right)$ and using Equation (11). Critically, however, the absorption systems which dominate the opacity, the so-called LLSs $\left(10^{17.2} \mathrm{~cm}^{-2} \leq \mathrm{N}_{\mathrm{HI}} \leq 10^{19} \mathrm{~cm}^{-2}\right)$ and saturated Ly $\alpha$ forest absorbers $\left(10^{14.5} \mathrm{~cm}^{-2} \leq \mathrm{N}_{\mathrm{HI}} \leq\right.$ $10^{17.2} \mathrm{~cm}^{-2}$ ), are those for which $N_{\mathrm{HI}}$ is most difficult to determine observationally. Extrapolations over this $N_{\mathrm{HI}}$ range are 


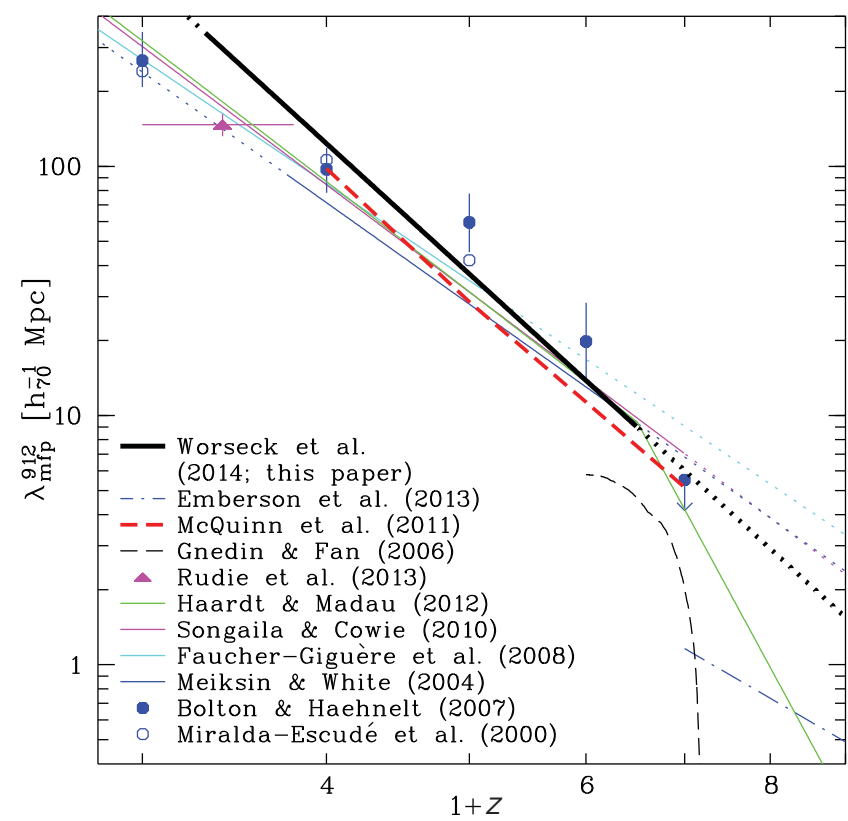

Figure 3. Summary of estimates for the proper mean free path at the Lyman limit, $\lambda_{\mathrm{LL}}(z)$. The results are based on stacked quasar spectra (Worseck et al. 2014), observations of discrete H Iabsorption systems (Faucher-Gigu et al. 2008b; Songaila \& Cowie 2010; Haardt \& Madau 2012; Rudie et al. 2013), optically thin IGM simulations using either semi-analytical (Miralda-Escudé et al. 2000; Bolton \& Haehnelt 2007c) or empirical (Meiksin \& White 2004) corrections for the abundance of self-shielded absorbers, and full radiative transfer models (Gnedin \& Fan 2006; McQuinn et al. 2011; Haardt \& Madau 2012; Emberson et al. 2013). Note that the estimates at $z \gtrsim 6$ are either extrapolations from data at $z<6$ or predictions from simulations. Reproduced from Figure 11 of Worseck et al. (2014) by permission of the authors.

therefore often employed. Previous studies have generally found $\beta_{\mathrm{z}} \simeq 1-3$ (note this is dependent on $N_{\mathrm{HI}}$ ) and $\beta_{\mathrm{N}} \simeq 1.3-1.7$ at $z>2$ (Tytler 1987; Petitjean et al. 1993; Storrie-Lombardi et al. 1994; Kim et al. 1997; Songaila \& Cowie 2010; Rudie et al. 2013).

However, recent work has demonstrated that the CDDF is not well described by a single power-law index $\beta_{\mathrm{N}}$, and may be better represented by a series of broken power laws (Prochaska, O'Meara, \& Worseck 2010; Kim et al. 2013; O'Meara et al. 2013). The expected clustering of LLS will also impact on $\lambda_{\text {mfp }}$ estimated from Equation (11), as this expression assumes the absorbers are Poisson distributed (Prochaska et al. 2014). This has led to the development of alternative approaches based on directly measuring the optical depth at the Lyman limit, $\tau_{\mathrm{LL}}$, using stacked spectra (Prochaska, Worseck, \& O'Meara 2009; O'Meara et al. 2013; Fumagalli et al. 2013; Worseck et al. 2014). Another approach used for estimating $\lambda_{\mathrm{mfp}}$ is to use hydrodynamical simulations which explicitly model the distribution of gas in the IGM, coupled with radiative transfer or analytical treatments for self-shielding (Miralda-Escudé, Haehnelt, \& Rees 2000; Meiksin \& White 2004; Gnedin \& Fan 2006; Bolton \& Haehnelt 2007c; McQuinn, Oh, \& Faucher-Giguère 2011;
Emberson, Thomas, \& Alvarez 2013; Sobacchi \& Mesinger 2014; Muñoz et al. 2016).

A selection of recent measurements and simulation predictions are shown in Figure 3, from Worseck et al. (2014). A recent analysis of $\tau_{\mathrm{LL}}$ in stacked quasar spectra by Worseck et al. (2014) provides a best-fitting evolution of $\lambda_{\mathrm{LL}}=37[(1+z) / 5]^{-5.4}$ proper Mpc at $2<z<5$. Using an alternative approach based on the observed incidence of LLSs, Songaila \& Cowie (2010) instead find a slightly shallower evolution with redshift, $\lambda_{\mathrm{LL}}=32[(1+z) / 5]^{-4.4}$ proper Mpc. Regardless of the precise normalisation and slope, however, these results indicate $\lambda_{\text {mfp }}$ evolves more quickly than expected for a population of absorbers with no intrinsic evolution. ${ }^{4}$

Consequently, there is significant evolution in the comoving number density and typical cross-section of Lyman limit absorbers toward higher redshift. This is directly related to the decline in the photoionisation rate at $z>5$; as the overall level of ionisation in the IGM drops, the typical density of LLS decreases, extending these optically thick systems out from the central regions of dark matter haloes to the surrounding filaments in the IGM. It has been suggested this coupling between $\lambda_{\text {mfp }}$ and $\Gamma_{\mathrm{HI}}$ (or equivalently, the sinks and sources of ionising photons in the IGM) naturally explains the flat evolution in $\Gamma_{\mathrm{HI}}$ from $2<z<5$ and its sudden decline approaching $z=6$ (McQuinn et al. 2011; Muñoz et al. 2016) when in contrast, the comoving ionising emissivity evolves slowly.

\subsection{The ionising emissivity}

Armed with observational determinations of $\Gamma_{\mathrm{HI}}(z)$ and $\lambda_{\mathrm{LL}}(z)$ at $z<6$, constraints on the integrated emissivity from ionising sources may now be obtained. If the typical mean free path for Lyman-limit photons is much smaller than the horizon scale, $\lambda_{\text {mfp }}(v) \ll c / H(z)$, photon redshifting effects are minimal and Equation (4) may be written as

$$
J(v) \simeq \frac{\epsilon(v) \lambda_{\mathrm{mfp}}(v)}{4 \pi} .
$$

This is referred to as the local source approximation (Madau et al. 1999; Schirber \& Bullock 2003). The proper specific emissivity, $\epsilon(v)$, is related to the comoving emissivity of ionising photons by

$$
\dot{N}_{\text {ion }}(z)=\frac{1}{(1+z)^{3}} \int_{v_{\mathrm{LL}}}^{\infty} \mathrm{d} \nu \frac{\epsilon(v)}{h_{\mathrm{P}} v} \simeq \frac{\epsilon_{\mathrm{LL}}}{h_{\mathrm{P}} \alpha_{\mathrm{s}}(1+z)^{3}},
$$

where the simplifying assumption of a power-law source spectrum, $\epsilon(v)=\epsilon_{\mathrm{LL}}\left(\nu / v_{\mathrm{LL}}\right)^{-\alpha_{\mathrm{s}}}$ is adopted. Substituting

\footnotetext{
${ }^{4}$ The expected evolution in absorption line number density with redshift is $\mathrm{d} n / \mathrm{d} z=c n_{\text {abs }}(z) \sigma_{\text {abs }}(z)(1+z)^{2} / H(z)$, where $n_{\text {abs }}(z)$ is the comoving number density of absorbers and $\sigma_{\mathrm{abs}}(z)$ is the physical absorber crosssection. At $z \geq 2, H(z) \propto(1+z)^{3 / 2}$ and thus $\mathrm{d} n / \mathrm{d} z \propto(1+z)^{1 / 2}$ if $n_{\text {abs }}(z)$ and $\sigma_{\text {abs }}(z)$ are held fixed (i.e. $\beta_{\mathrm{z}}=0.5$ ). Hence from Equations (11), for a non-evolving population of Poisson distributed absorbers, the (proper) mean free path $\lambda_{\mathrm{mfp}} \propto(1+z)^{-3}$.
} 
Equations (5), (12) and (11) into this expression gives

$$
\dot{N}_{\text {ion }}(z) \simeq \frac{\Gamma_{\mathrm{HI}}(z)}{\sigma_{\mathrm{LL}} \lambda_{\mathrm{LL}}(z)} \frac{\left(\alpha_{\mathrm{bg}}+3\right)}{\alpha_{\mathrm{s}}} \frac{1}{(1+z)^{3}},
$$

where we may also identify $\alpha_{\mathrm{bg}}=\alpha_{\mathrm{s}}-3\left(\beta_{\mathrm{N}}-1\right)$ if the CDDF is described by a single power-law index (but see Section 2.4).

Equation (14) may be used to estimate the total ionising emissivity in the IGM, given observational determinations of $\Gamma_{\mathrm{HI}}(z)$ and $\lambda_{\mathrm{LL}}(z)$. Values for $\alpha_{\mathrm{s}}$ will depend on the sources which dominate the UVB. For AGN, $\alpha_{\mathrm{s}}=1.5-1.8$ (Telfer et al. 2002; Stevans et al. 2014), whereas for star-forming galaxies $\alpha_{\mathrm{s}}=1-3$ (e.g. Leitherer et al. 1999; Eldridge \& Stanway 2012). Note, however, the local source approximation ignores the cosmological redshifting of ionising photons to frequencies $v<v_{\mathrm{LL}}$, and thus underestimates $\dot{N}_{\text {ion }}$ for a given $\Gamma_{\mathrm{HI}}$ by $\sim 50 \%(\sim 10 \%)$ at $z=2(z=5)$ (Becker $\&$ Bolton 2013). Modelling $\lambda_{\text {LL }}$ with Equation (11) and adopting a single power-law source spectrum are further simplifications; these enable analytical forms for the integrals, but more detailed treatments often require these equations to be solved numerically.

Recent inferences are consistent with $\dot{N}_{\text {ion }} \sim$ $10^{51} \mathrm{~s}^{-1} \mathrm{Mpc}^{-3}$ at $2<z<6$ (Becker \& Bolton 2013). These are around a factor of 2 higher than earlier estimates (Bolton \& Haehnelt 2007c; Kuhlen \& Faucher-Giguère 2012), largely due to improved constraints for the mean free path at $z>4$ and the temperature of the IGM at $2<z<5$. The emissivity is also often expressed as the number of ionising photons emitted per hydrogen atom over a Hubble time, $\dot{n}_{\text {ion }}=\dot{N}_{\text {ion }}(1+z)^{3} / \bar{n}_{\mathrm{H}} H(z)$, where $t_{\text {age }} \simeq 2 / 3 H(z)$ is the age of the Universe at $z \geq 2$. Miralda-Escude (2003) first pointed out that the emissivity at $z=4$ corresponds to $\dot{n}_{\text {ion }} \leq 7$, which strictly limits the amount by which it may decline at $z>4$ if reionisation is to complete by $z=6$. Similar conclusions were reached by Meiksin (2005), and later Bolton \& Haehnelt (2007c), who extended the analysis to $z=6$, finding $\dot{n}_{\text {ion }} \leq 2-5$.

In comparison, recent cosmological radiative transfer simulations indicate the reionisation of the IGM requires $\gtrsim 2-3$ ionising photons per atom to counterbalance radiative recombinations (Finlator et al. 2012; So et al. 2014). The close correspondence between this number and $\dot{n}_{\text {ion }}$ indicates reionisation may be a 'photon-starved' process; there are only just enough photons present to reionise the IGM at $z \simeq 6$. Consequently, the metagalactic ionising emissivity at $z \leq 6$ is an important observational constraint which viable reionisation models at $z>6$ must anchor to Pritchard, Loeb, \& Wyithe (2010), Alvarez, Finlator, \& Trenti (2012), Finlator et al. (2012), Mitra, Ferrara, \& Choudhury (2013), and Fontanot et al. (2014).

\subsection{The contribution from galaxies and AGN}

The ionising emissivity inferred from the Ly $\alpha$ forest may be directly compared to estimates based on the observed popu- lation of sources at high redshift. The power of this approach is that the metagalactic emissivity represents the combined photon output from all ionising sources, irrespective of uncertain physics at galactic scales. If the UV luminosity function, $\phi\left(L_{\mathrm{UV}}, z\right)$, for a given source population is known, the proper UV specific emissivity ${ }^{5}$ is given by

$$
\epsilon_{\mathrm{UV}}=(1+z)^{3} \int_{L_{\min }}^{\infty} \mathrm{d} L_{\mathrm{UV}} L_{\mathrm{UV}} \phi\left(L_{\mathrm{UV}}, z\right)
$$

The luminosity function for LBGs is typically parameterised as a Schechter (1976) function, whereas AGN are often described by a double power law (e.g. Ueda et al. 2003; Richards et al. 2006; Croom et al. 2009). The proper emissivity at the Lyman limit is then $\epsilon_{\mathrm{LL}}=f_{\text {esc }} f_{\mathrm{LL}} \epsilon_{\mathrm{UV}}$, where $f_{\mathrm{LL}}=\epsilon_{\mathrm{LL}, \text { int }} / \epsilon_{\mathrm{UV}}$ is the ratio of the intrinsic Lyman limit and $\mathrm{UV}$ emissivities, and $f_{\mathrm{esc}}$ is the (average) fraction of ionising photons which escape the local interstellar medium. For AGN, a broken power-law approximation is often used to estimate $f_{\mathrm{LL}}$ and $f_{\text {esc }}=1$ is typically assumed (e.g. Madau et al. 1999), while for high-redshift galaxies stellar population synthesis models are employed to obtain $f_{\mathrm{LL}}$ and $f_{\text {esc }}$ is a free parameter. The spectral properties of galaxies may also be constrained further with observed UV spectral slopes (Finkelstein et al. 2012; Dunlop et al. 2013; Robertson et al. 2013; Bouwens et al. 2014; Duncan \& Conselice 2015). The comoving emissivity of ionising photons is then obtained using Equation (13).

Figure 4 displays $\dot{N}_{\text {ion }}$ inferred from the UV luminosity densities reported by Oesch et al. (2013), Bouwens et al. (2015), and Finkelstein et al. (2015), assuming ${ }^{6}$ $f_{\mathrm{LL}}=0.25 \pm 0.10, f_{\text {esc }}=0.3 \pm 0.2$ and a power-law spectral index below the Lyman limit, $\alpha_{\mathrm{s}}=2 \pm 1$ (e.g Becker \& Bolton 2013). These data are based on recent measurements of the LBG luminosity function to a limiting absolute magnitude of $M_{\mathrm{UV}} \simeq-17$ at $4<z<10.5$, and are compared to independent measurements of $\dot{N}_{\text {ion }}$ from the Ly $\alpha$ forest at $2<z<5$, along with models for the expected emissivity from AGN (blue curves) and star-forming galaxies (red curves). The dotted curves display the comoving emissivity required to balance radiative recombinations in the IGM (Madau et al. 1999), where

$$
\dot{N}_{\text {rec }}=\bar{n}_{\mathrm{H}}^{2}\left(1+\chi_{\mathrm{He}}\right) C_{\mathrm{HII}}(z) \alpha_{\mathrm{HII}}(T)(1+z)^{3} .
$$

Here, $C_{\mathrm{HII}}=\left\langle n_{\mathrm{HII}}^{2}\right\rangle / \bar{n}_{\mathrm{HII}}^{2}$ is the clumping factor. This takes into account the enhancement in the volume averaged recombination rate due to small scale, dense structures. Typical values for the ionised, low-density IGM obtained from numerical simulations are $C_{\mathrm{HII}} \simeq 1-5$ (Pawlik, Schaye, \& van

\footnotetext{
${ }^{5}$ This quantity is typically described in the observational literature as a comoving UV luminosity density, $\rho_{\mathrm{UV}}=\epsilon_{\mathrm{UV}} /(1+z)^{3}$ with units $\mathrm{erg} \mathrm{s}^{-1} \mathrm{~Hz}^{-1} \mathrm{Mpc}^{-3}$. This is often subsequently converted to a star formation rate density (in units of $\mathrm{M}_{\odot} \mathrm{yr}^{-1} \mathrm{Mpc}^{-3}$ ), using the relation $\rho_{\mathrm{SFR}}=1.25 \times 10^{-28} \rho_{\mathrm{UV}}$ (Kennicutt 1998; Finkelstein et al. 2015). This assumes a Salpeter initial mass function and a constant star formation rate. ${ }^{6}$ Note the $\dot{N}_{\text {ion }}$ measurements from the Ly $\alpha$ forest are independent of these (uncertain) assumptions and are thus complementary to constraints from the UV luminosity density.
} 


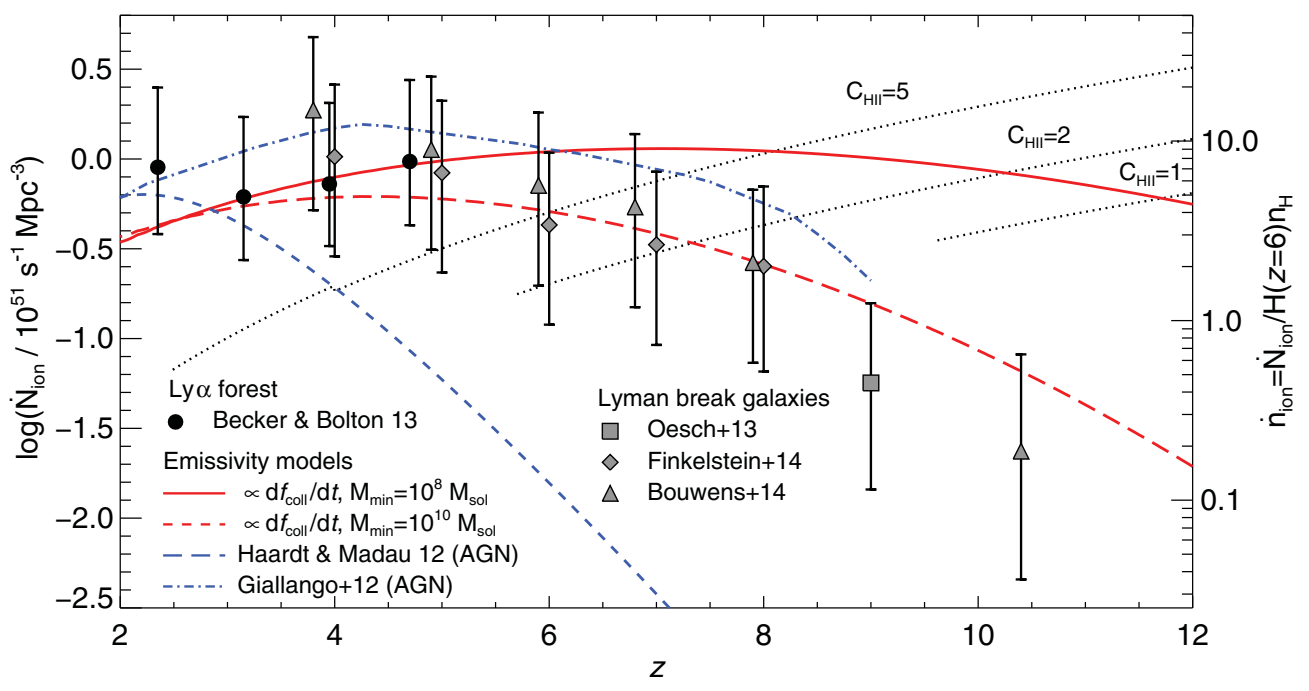

Figure 4. The comoving emissivity of ionising photons with redshift. The filled circles correspond to estimates from the Ly $\alpha$ forest opacity (Becker \& Bolton 2013) (see their Table 3 for a full breakdown of the systematic uncertainties), and the grey symbols show results derived here from the comoving UV luminosity density, $\rho_{\mathrm{UV}}$, of Lyman break galaxies (Oesch et al. 2013; Finkelstein et al. 2015; Bouwens et al. 2015). The error bars on these data are computed by adding the published uncertainties on $\rho_{\mathrm{UV}}$ in quadrature with the systematic uncertainties $f_{\mathrm{LL}}=0.25 \pm 0.10$, $f_{\text {esc }}=0.3 \pm 0.2$ and $\alpha_{\mathrm{s}}=2 \pm 1$ (see the text and Equations (13) and (15) for details). The red curves display a model for the emissivity from star-forming galaxies, where the ionising efficiency is fixed at $\zeta=25$ independent of redshift. Here, $\dot{N}_{\text {ion }}$ is proportional to the rate of change of the collapsed fraction of matter in haloes with $M_{\min } \geq 10^{8} M_{\odot}$ (solid curve) and $M_{\min } \geq 10^{10} M_{\odot}$ (long-dashed curve). The blue short-dashed and dot-dashed curves show the AGN emissivity models from Haardt \& Madau (2012) and Giallongo et al. (2012), respectively. The dotted curves correspond to the ionising emissivity required to balance recombinations for three different hydrogen clumping factors, $C_{\mathrm{HII}}=1$, 2 and 5; these are truncated where the recombination timescale exceeds the age of the Universe.

Scherpenzeel 2009; Finlator et al. 2012; Shull et al. 2012; Jeeson-Daniel et al. 2014; So et al. 2014; Kaurov \& Gnedin 2014). Alternatively, assuming the IGM is in ionisation equilibrium (cf. Equation 6), then Equation (14) and (16) may be equated and rearranged for $C_{\mathrm{HII}}$. Inserting observational estimates for $\Gamma_{\mathrm{HI}}$ and $\lambda_{\mathrm{LL}}$ yields $C_{\mathrm{HII}} \simeq 2-3$ (Bolton \& Haehnelt 2007c; McQuinn et al. 2011).

Figure 4 demonstrates that the observed population of LBGs at $z<6$ is consistent with the emissivity inferred from the Ly $\alpha$ forest, although the lack of evolution in the latter from $z=4-5$ implies that the escape fraction of ionising photons, $f_{\text {esc }}$, may be increasing toward higher redshift (e.g. Alvarez et al. 2012; Ciardi et al. 2012; Kuhlen \& FaucherGiguère 2012; Shull et al. 2012; Ferrara \& Loeb 2013). However, the emissivity from these galaxies rapidly drops below the critical rate required to balance recombinations at $z \gtrsim 6$, indicating the observed sources are insufficient to drive reionisation to completion. There are three ways to resolve this dilemma: (i) there are many more faint galaxies below the detection limit which contribute to the total photon budget; (ii) the ionising efficiency (the product of $f_{\mathrm{LL}}$ and $f_{\mathrm{esc}}$ ) of these galaxies increases significantly toward higher redshift, or (iii) there is another population of sources which produce ionising photons. A combination of all three may also be plausible.

To illustrate this further, the red curves in Figure 4 display a simple model for the expected emissivity from star-forming galaxies, where

$$
\dot{N}_{\text {ion }}(z)=\zeta(z) \bar{n}_{\mathrm{b}} \frac{\mathrm{d} f_{\text {coll }}\left(>M_{\text {min }}, z\right)}{\mathrm{d} t},
$$

(e.g Pritchard et al. 2010; Wyithe et al. 2010; Sobacchi \& Mesinger 2014). Here, $f_{\text {coll }}$ is the collapsed fraction of matter in haloes above a minimum mass threshold $M_{\min }$ (Sheth \& Tormen 2002), and $\zeta(z)=f_{\star} f_{\mathrm{esc}} N_{\gamma}$ is the ionising efficiency of star-forming galaxies (assumed to be constant, $\zeta=25$, in Figure 4). This ionising efficiency is itself a function of three uncertain parameters, the star formation efficiency $f_{\star}$, the number of ionising photons produced per baryon within stars, $N_{\gamma}$, and the escape fraction. ${ }^{7}$

Considering all haloes above the mass threshold at which atomic cooling ceases to be efficient, $M_{\min } \simeq 10^{8} \mathrm{M}_{\odot}$, produces an emissivity in excess of that observed from galaxies with $M_{\mathrm{UV}} \lesssim-17$ at $z \gtrsim 6.5$. Better agreement may be obtained by extrapolating the observed faint end of the luminosity function to $M_{\mathrm{UV}} \simeq-10$ with Equation (15); faint, high-redshift dwarf galaxies are therefore widely postulated as likely candidates for making up the observed ionising photon shortfall at $z>6$ (Trenti et al. 2010; Robertson et al. 2013, 2015; Fontanot et al. 2014; Bouwens et al. 2015). This

\footnotetext{
${ }^{7}$ Illustrative values are $N_{\gamma}=4600$ (17 500) for a Salpeter (top-heavy) IMF (Wyithe et al. 2010), $f_{\star}=0.01-0.1$ (Behroozi, Wechsler, \& Conroy 2013) and $f_{\text {esc }}=0.05-0.5$ (Wise et al. 2014), where the latter two values will vary with halo mass.
} 
illustrates the important role of IGM studies in providing a complete census of early galaxy populations; many of the ionising sources are too faint to detect individually, but we can nevertheless observe their combined impact on the surrounding intergalactic gas. Alternatively, as discussed above the ionising efficiency may increase with redshift, if e.g. $f_{\text {esc }}$ evolves with redshift. However, direct measurements of $f_{\text {esc }}$ approaching reionisation are prohibited by the high opacity of the intervening IGM, and searches for analogues at lower redshift indicate the amount of Lyman continuum radiation escaping from galaxies is modest (e.g. Vanzella et al. 2010; Boutsia et al. 2011; Nestor et al. 2013; Mostardi et al. 2013).

Lastly, it remains possible that other sources contribute to the total ionising emissivity. The most natural candidate are AGN, with hard, non-thermal spectra which produce an abundance of ionising photons. The blue curves in Figure 4 display two very different models for the predicted contribution of AGN to $\dot{N}_{\text {ion }}$. The lower estimate is from the UVB model of Haardt \& Madau (2012), which is based on the bolometric luminosity functions presented by Hopkins et al. (2007) at $0<z<6$. There is a sharp drop in $\dot{N}_{\text {ion }}$ at $z>4$ as the number density of sources falls, implying that AGN are unlikely to be the dominant sources powering reionisation (see also Cowie, Barger, \& Trouille 2009). On the other hand, semi-analytical modelling by Giallongo et al. (2012), incorporating faint $\left(M_{\mathrm{UV}} \simeq-18.5\right) \mathrm{AGN}$, produces $\dot{N}_{\text {ion }}$ estimates up to two orders of magnitude larger. If such faint AGN are widespread, these could provide almost all the ionising photons required to complete reionisation. However, uncertainties associated with the number of faint AGN at $z>4$ remain considerable, leaving their precise contribution to the ionising photon budget a matter of debate (e.g. Glikman et al. 2011; Masters et al. 2012; Giallongo et al. 2015). The number and relative contribution of AGN to the UVB must furthermore remain consistent with upper limits on the unresolved X-ray background (Dijkstra, Haiman, \& Loeb 2004a; Haardt \& Salvaterra 2015) and the spectral shape of the UVB inferred from metal ion absorption lines (Section 3) at $2<z<4$ (Agafonova et al. 2007; Fechner 2011; Boksenberg \& Sargent 2015). Lastly, the double reionisation of helium is expected to compete around $z \simeq 2$ 3 , based on observations of the intergalactic He Ilopacity (Shull et al. 2010; Worseck et al. 2011) and the IGM temperature (Schaye et al. 2000; Lidz et al. 2010; Becker et al. 2011a). This is thought to be driven by the hard photons emitted by AGN (Furlanetto \& Oh 2008; McQuinn et al. 2009). Consequently, their contribution at $z>4$ must avoid a premature end to He IIreionisation (McQuinn 2012).

\subsection{Spatial fluctuations in the UVB}

Finally in this section, we turn to consider spatial inhomogeneities in the UVB. In addition to the average photoionisation rate determined from the mean transmission of the Ly $\alpha$ forest, one can also examine spatial fluctuations in $\Gamma_{\mathrm{HI}}$ using the line-of-sight variation in the transmission as a function of redshift (Fan et al. 2006). If reionisation is incomplete, this may boost the amplitude of spatial variations in the transmission, with some lines-of-sight crossing mostly through ionised bubbles, and others intersecting multiple neutral regions in the IGM (Wyithe \& Loeb 2006). Note, however, the scatter in the average transmission may also be large shortly after reionisation completes, when the local mean free path to ionising photons is similar to the typical separation between (clustered) sources. In this scenario, the mean free path itself may vary spatially; even though the IGM is highly ionised, the UVB will exhibit significant spatial fluctuations (e.g. Zuo 1992; Meiksin \& White 2004; Mesinger \& Furlanetto 2009).

Importantly, these fluctuations potentially yield information on the timing of patchy reionisation and the distribution of the ionising sources; the scatter in the average transmission may be an interesting diagnostic in this respect. However, fluctuations owing to spatial variations in the underlying density field may also contribute significantly to the observed scatter (Lidz et al. 2006). The aliasing of transverse, small-scale density fluctuations in three dimensions to larger scales along the one-dimensional quasar sightlines can further obscure effects from incomplete reionisation (cf. Kaiser \& Peacock 1991).

Nevertheless, recent work indicates that the observed scatter is indeed larger than expected in uniform UVB models, especially at $z \gtrsim 5.5$ (Becker et al. 2015). This finding is driven in part by the discovery of a contiguous $110 \mathrm{~h}^{-1}$ comoving Mpc dark region in the $z \sim 5.7$ Ly $\alpha$ forest. Such a large scatter in the mean transmission is seen at $z<6$ in radiative transfer simulations where reionisation competes between $z=6$ and 7 (Gnedin 2014), and may be a natural consequence of spatial variations in the mean free path at the tail-end of reionisation (Furlanetto \& Oh 2005). It has also been recently suggested the fluctuations may instead be indicative of photoionisation by rare, bright quasars (Chardin et al. 2015). Alternatively, reionisation may be incomplete at $z \sim 5.5--6$ (Lidz et al. 2007; Mesinger 2010, and see Section 4.2). Consequently, while these observations are highly suggestive, we have yet to determine their full implications for the reionisation history of the Universe.

\section{METAL ABSORPTION LINES}

Metal absorption lines complement the Ly $\alpha$ forest as probes of the high-redshift Universe in that they reflect the chemical enrichment and ionisation state in the regions in and around galaxies. The overall abundance of metals reflects the integrated global star formation history, while the number densities, ionic ratios, and kinematic profiles of absorbers contain information on the mechanisms by which galaxies produce and expel metals (i.e., feedback mechanisms), the stellar populations that produced the metals, and the ionisation state of the metal-enriched gas. Metal absorbers are 
particularly valuable tracers of galaxies during reionisation, as they can arise from faint galaxies that are below the detection thresholds of direct galaxy surveys. In this section, we give an overview of recent results on metal lines over $z \sim 5--6$. We first describe the observations, then examine what metal absorbers tell us about galaxies and their environments at these redshifts.

\subsection{Observations of metal lines near $z \sim 6$}

Studies of metal lines near reionisation have tended to focus on three types of absorbers: highly ionised systems traced by $\mathrm{C}$ IV and/or Si IV (which we will generally refer to as C IV systems); low-ionisation systems traced by O I, C II, $\mathrm{Si}$ II, and Fe II (which we will refer to as O I systems); and $\mathrm{Mg}$ II systems, which can trace a range of ionisation states ${ }^{8}$. Studies of C IV (rest-frame $\lambda=1548,1551 \backslash \mathrm{AA}$ ) and Mg II ( $\lambda=2796,2804 \backslash$ AA), which are observed in the infrared at $z>5.5$ and 2.6, respectively, have particularly benefited from the substantial increase in infrared spectroscopic sensitivity recently provided by the X-Shooter spectrograph on the Very Large Telescope (D'Odorico et al. 2006), and the Folded-port Infrared Echellette (FIRE) spectrograph on Magellan (Simcoe et al. 2013). We will begin by describing the observations of C IV, O I, and Mg II absorbers separately, although the reader should bear in mind that these often trace different components of the same system. To date, quasars have been used for systematic surveys of metals at $z>5$; however, gamma ray burst (GRB) afterglows have also started to yield samples of metal lines at these redshifts (e.g. Chornock et al. 2013; Castro-Tirado et al. 2013; Hartoog et al. 2015).

\subsubsection{Metrics}

Before describing the observations, we first introduce some of the metrics used to quantify metal absorption line samples. An absorber population can be characterised by its column density distribution, $f(N)=\partial^{2} n / \partial N \partial X$, which gives the number of systems per unit column density, $N$, and absorption path length interval $X$ (see also Section 2.4). The absorption path length interval is related to the redshift interval by

$$
\frac{\mathrm{d} X}{\mathrm{~d} z}=(1+z)^{2} \frac{H_{0}}{H(z)},
$$

Bahcall \& Peebles (1969), and has the useful property that a population of sources with a fixed physical cross-section will have a constant number density per unit $X$.

Two quantities related to $f(N)$ that are often quoted for metal absorbers are the line-of-sight number density, $\mathrm{d} n / \mathrm{d} X$ (or $\mathrm{d} n / \mathrm{d} z$ ), and the comoving mass density. The total mass density of an ion can be expressed as a fraction of $\rho_{\text {crit }}$, the

\footnotetext{
${ }^{8}$ Throughout this review, we will follow the common convention in quasar absorption line literature of referring to an ion by its absorption spectrum, e.g. C IV for $\mathrm{C}^{3+}$ ions.
}

critical density at $z=0$,

$$
\Omega_{\text {ion }}=\frac{H_{0} m_{\text {ion }}}{c \rho_{\text {crit }}} \int_{N_{\text {min }}}^{N_{\text {max }}} N_{\text {ion }} f\left(N_{\text {ion }}\right) \mathrm{d} N_{\text {ion }},
$$

which can be approximated as

$$
\Omega_{\text {ion }}=\frac{H_{0} m_{\text {ion }}}{c \rho_{\text {crit }}} \frac{\sum N_{\text {ion }}}{\Delta X} .
$$

Here, $N_{\text {ion }}$ is the column density of an absorber and $\Delta X$ is the total survey path length.

\subsubsection{CIV}

The $\mathrm{C}$ IV doublet is perhaps the most commonly used absorption line tracer of metals due to its sensitivity to enriched, highly ionised gas. The first $\mathrm{C}$ IV measurements at $z \sim 6$ were made by Simcoe (2006) and Ryan-Weber, Pettini, \& Madau (2006), with larger samples and improved sensitivity provided by Becker, Rauch, \& Sargent (2009), Ryan-Weber et al. (2009), Simcoe et al. (2011), and D'Odorico et al. (2013). At present, $13 z \sim 6$ quasars have been surveyed for $\mathrm{C}$ IV along their line of sight. An example system at $z=5.9$ is shown in Figure 5, which in this case displays both $\mathrm{C}$ IV and $\mathrm{Si}$ IV absorption.

While precise fits to $f\left(N_{\mathrm{CIV}}\right)$ at $z \sim 6$ remain difficult due to the small number of absorbers, D'Odorico et al. (2013) find that the $z>5.3$ data can be fit with a power law in $N_{\text {CIV }}$ with a slope similar to that for C IV populations at lower redshifts, but with a normalisation that is a factor of 2 to 4 lower (see also Becker et al. 2009).

Figure 6 shows the evolution for $\Omega_{\mathrm{CIV}}$ over $0<z<6$. In general, the mass density of C IV increases towards lower redshifts, which is consistent with the buildup of highly ionised metals in the circumgalactic medium of galaxies due to processes such as outflows (e.g. Oppenheimer \& Davé 2006). Changes in the ionisation state of the metal-enriched gas will also affect the $\mathrm{C}$ IV statistics, however. The ionisation state of C IV-selected gas will tend to evolve due to changes in density and/or the UVB, such that the fraction of carbon in these systems traced by $\mathrm{C}$ IV will change with redshift. The evolution of $\Omega_{\mathrm{CIV}}$ is therefore the product of increasing metal enrichment and changes in the ionisation state of the metal-enriched gas. Generally speaking, C IV becomes a preferred ionisation state at lower overdensities towards higher redshifts (e.g. Oppenheimer \& Davé 2006; Oppenheimer, Davé, \& Finlator 2009). It is noteworthy that $\Omega_{\mathrm{CIV}}$ appears to increase by a factor of 2 to 4 from $z \sim 6$ to 5 . This suggests a substantial buildup of metal-enriched regions around galaxies over this interval, although the rate of increase may depend partly on the range of column densities being considered (D'Odorico et al. 2013).

\section{$3.1 .3 O_{I}$}

Low-ionisation metal lines are potentially powerful probes of the high-redshift Universe in multiple ways. First, they trace the dense gas in and around galaxies, offering insights into the interstellar media of these objects analogous to the ways 

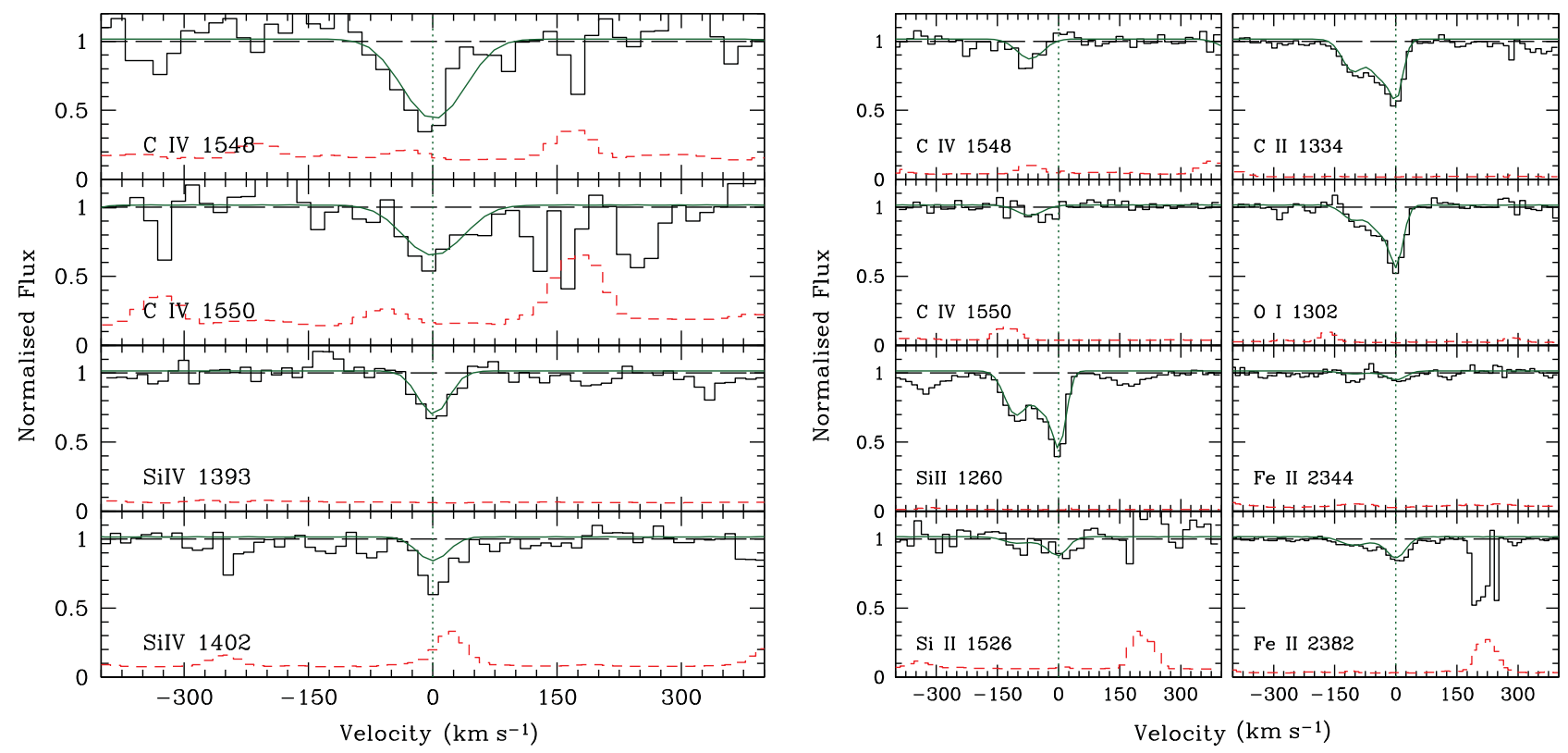

Figure 5. Examples of $\mathrm{C}$ IV (left) and low-ionisation (right) metal absorption line systems observed with VLT/X-Shooter, at $z=5.92$ and 5.79, respectively. The transitions for a given absorber have been shifted onto a common velocity scale. Histograms show the continuum-normalised flux, while the dashed lines are the flux uncertainty. Solid lines are Voigt profile fits. Reproduced from Figures 5 and 17 of D'Odorico et al. (2013) with permission of the authors.

in which damped Ly $\alpha$ systems (DLAs) trace the kinematics and composition of lower-redshift galaxies (e.g. Wolfe, Gawiser, \& Prochaska 2005). Second, because the metals in these systems are often dominated by a single ionisation state, determining abundances is relatively straightforward. Finally, if the last regions of the IGM to be reionised are metal enriched, then they may give rise to a 'forest' of absorption lines such as $\mathrm{O}$ I and $\mathrm{C}$ II that can be detected in quasar spectra (e.g. Oh 2002; Furlanetto \& Loeb 2003).

Metal-enriched gas where the hydrogen is largely neutral is traced by lines such as $\mathrm{O}$ I, C II, Si II, and Fe II. O I is particularly useful for studying neutral gas since the first ionisation potential of oxygen and hydrogen are very similar, and due to charge exchange, $n\left(\mathrm{O}^{+}\right) / n(\mathrm{O}) \simeq n\left(\mathrm{H}^{+}\right) / n(\mathrm{H})$ over a wide range of physical conditions (e.g. Osterbrock \& Ferland 2006). Elements where the first ionisation potential is significantly less than $13.6 \mathrm{eV}$, such as carbon, silicon, and iron, are not shielded by atomic hydrogen, and therefore appear as singly ionised species in otherwise 'neutral' gas. At $z<5$, low-ionisation systems are typically found by their strong, often damped, hydrogen Ly $\alpha$ lines. At higher redshifts, however, the growing saturation of the Ly $\alpha$ forest makes identifying individual Ly $\alpha$ absorbers difficult, and metal systems must be identified using 'pseudo multiplets' of lines redward of Ly $\alpha$, such as O I $\lambda 1302$; $\mathrm{C}$ II $\lambda 1334$; and Si II $\lambda 1260, \lambda 1304$, and $\lambda 1526$.

Becker et al. (2006, 2011b) searched for low-ionisation systems over $5.3<z_{\text {abs }}<6.4$. In 17 lines of sight, they find 10 systems with C II and Si II, nine of which also contain O I. Infrared spectra have been used to obtain $\mathrm{Fe}$ II for many of these systems (Becker et al. 2012; D'Odorico et al. 2013). Among the nine O I systems, the ratios of O I, C II, Si II, and Fe II column densities are reasonably constant, which suggests that neither ionisation corrections nor dust depletion are large factors, as these would tend to introduce scatter. This supports the expectation that $\mathrm{O}$ I systems trace predominantly neutral gas, and are therefore the analogues of lower-redshift DLAs $\left(N_{\mathrm{HI}} \geq 10^{20.3} \mathrm{~cm}^{-2}\right)$ (e.g. Wolfe et al. 2005) and sub-DLAs $\left(10^{19} \mathrm{~cm}^{-2}<N_{\mathrm{HI}}<10^{20.3} \mathrm{~cm}^{-2}\right.$ ) (e.g. Dessauges-Zavads et al. 2003). ${ }^{9}$ It is therefore reasonable to compare $\mathrm{O}$ I systems at $z>5$ to these systems, although it should be emphasised that $\mathrm{H}$ I-selected systems with $N_{\mathrm{HI}}>10^{19} \mathrm{~cm}^{-2}$ may not represent a complete census of $\mathrm{O}$ I systems at lower redshifts.

In terms of number density, Becker et al. (2011b) find $\mathrm{d} n / \mathrm{d} X=0.25_{-0.13}^{+0.21}$, which is similar to the combined number density of DLAs and sub-DLAs over $3<z<5$ (Péroux et al. 2005; O'Meara et al. O'Meara et al.; Prochaska \& Wolfe 2009; Noterdaeme et al. 2009; Guimarães et al. 2009; Crighton et al. 2015). At least one of the O I systems lies close to the detection limit of the existing data, however (Becker et al. 2011b), so it is possible that the number density of weak low-ionisation systems $\left(N_{\mathrm{OI}} \lesssim 10^{13.5} \mathrm{~cm}^{-2}\right.$, $N_{\text {CII }} \lesssim 10^{13.0} \mathrm{~cm}^{-2}$ ) may be larger. The observed mass density of $\mathrm{O}_{\mathrm{I}}$ in systems at $z \sim 6$ can be directly computed using Equation (20) because the lines are typically unsaturated, and so have measurable column densities. At lower

${ }^{9}$ Indeed, Becker et al. (2011b) find that the metal line kinematics of $\mathrm{O}_{\mathrm{I}}$ systems at $z \sim 6$ are comparable to those of lower-redshift DLAs and sub-DLAs, although the lines strengths are weaker. 


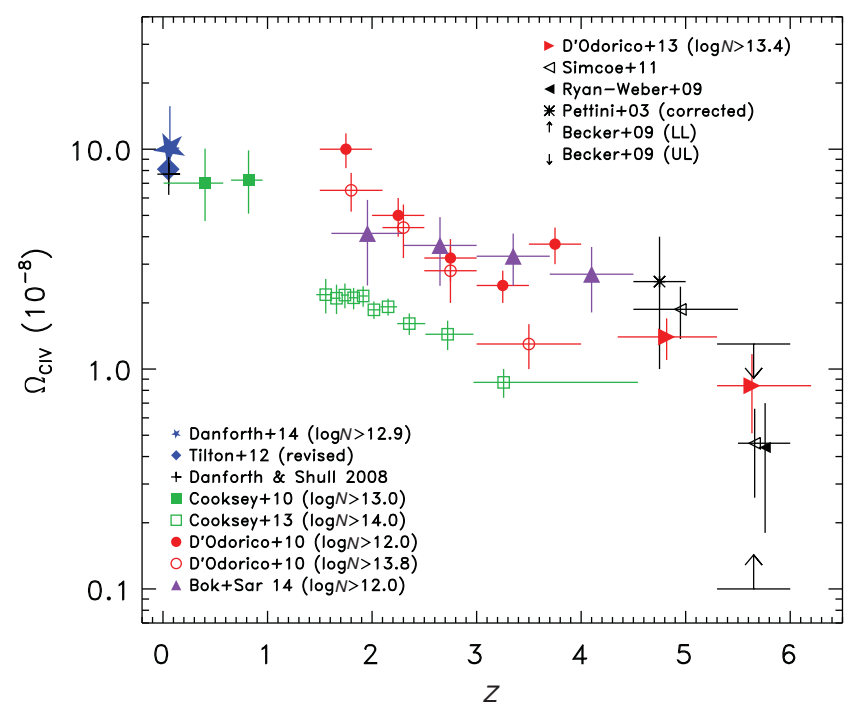

Figure 6. Evolution of the comoving mass density of C IV, expressed as a fraction of the critical density. Results from the literature are plotted with symbols indicated in the figure legend (Pettini et al. 2003; Danforth \& Shull 2008; Becker et al. 2009; Ryan-Weber et al. 2009; Cooksey et al. 2010, 2013; D'Odorico et al. 2010, 2013; Simcoe et al. 2011; Tilton et al. 2012; Danforth et al. 2014; Boksenberg \& Sargent 2015, where 'Bok+Sar 14' in the caption refers to Boksenberg \& Sargent 2015). Note that some of the variation in these results is due to differences in the column density range over which $\Omega_{\mathrm{CIV}}$ is integrated. Reproduced from Figure 1 of Shull, Danforth, \& Tilton (2014) by permission of the authors and the AAS.

redshifts, $\mathrm{O}$ I is generally saturated; however, the total mass density can be estimated by combining measurements of $\Omega_{\mathrm{HI}}$ in DLAs, which dominate the neutral gas budget (e.g. Noterdaeme et al. 2009; Crighton et al. 2015) with the column density-weighted mean DLA metallicity (e.g. Rafelski et al. 2014). Figure 7 shows $\Omega_{\mathrm{OI}}$ at $z \sim 6$ computed from the Becker et al. (2011b) measurements, along with an estimates over $2<z<4.5$ based on the fit to $\Omega_{\mathrm{HI}}(z)$ in Crighton et al. (2015) and a fit to the mean metallicity evolution at $z<4.7$ from Rafelski et al. (2014). A separate estimate at $z=4.85$ is also given based on discrete $\Omega_{\mathrm{HI}}$ and mean DLA metallicity measurements by these authors near $z \sim 5$.

While these mass density estimates should be treated with caution, they broadly suggest a substantial buildup of metals in the low-ionisation phase over $4.5 \lesssim z \lesssim 6$, followed by a more gradual buildup down to $z \sim 2$. Given that $\Omega_{\mathrm{HI}}$ decreases with time (Prochaska \& Wolfe 2009; Noterdaeme et al. 2009; Crighton et al. 2015), and that the number density of low-ionisation absorbers does not change rapidly (see above), the increase in the mass density of low-ionisation metals may be largely driven by an increase in the (lowionisation phase) metal content of the absorbers.

\subsubsection{Mg II}

The final absorption-line probe of metals at $z>5$ is the $\mathrm{Mg}$ II doublet, which can trace both neutral and ionised gas phases. The first survey for $\mathrm{Mg}$ II over $2.5<z<6$ was conducted

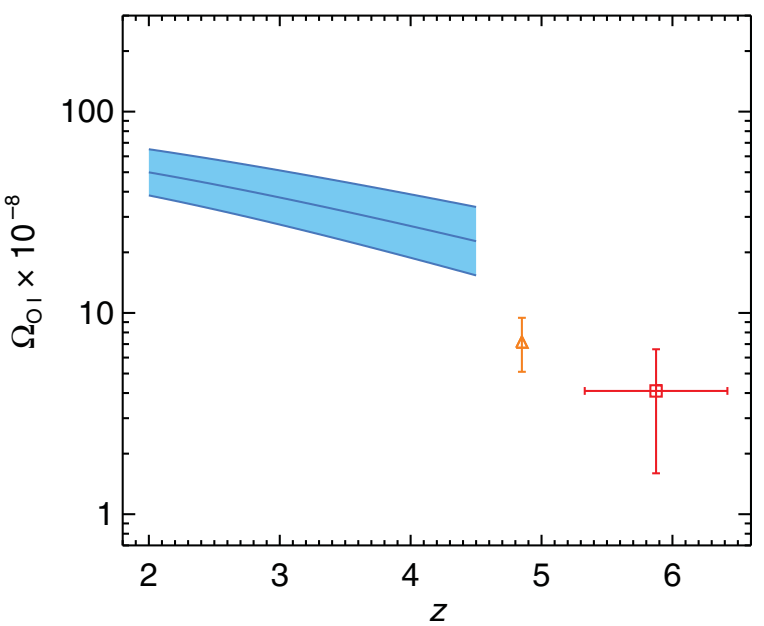

Figure 7. Evolution of the comoving mass density of $\mathrm{O} \mathrm{I}$, expressed as a fraction of the critical density. The open square is derived from measurements of individual absorbers in Becker et al. (2011b). The shaded region is an estimate based on fits to $\Omega_{\mathrm{HI}}(z)$ for DLAs from Crighton et al. (2015) and the mean DLA metallicity from Rafelski et al. (2014). The open triangle at $z=4.85$ is calculated from discrete $\Omega_{\mathrm{HI}}$ and mean DLA metallicity values from these works near $z \sim 5$.

by Matejek \& Simcoe (2012), followed by an expanded sample from Chen et al. (in preparation), both using FIRE data. Column densities of strong $\mathrm{Mg}$ II systems are often difficult to measure due to saturation effects, and it is therefore common to quantify these systems in terms of the rest-frame equivalent width, $W_{\mathrm{r}}$, of the $2796 \backslash \mathrm{AA}$ line. Incidence rates of $\mathrm{Mg}$ II systems in different ranges in $W_{\mathrm{r}}$ are shown in Figure 8. The $z>2$ data are compared to lower-redshift measurements using Sloan Digital Sky Survey (SDSS) data from Nestor et al. (2005) and Seyffert et al. (2013). For weak systems, the higher-resolution FIRE spectra tend to be more sensitive than the larger SDSS samples. Incompleteness corrections are therefore important in comparing these samples, particularly for $W_{\mathrm{r}}<1 \backslash \mathrm{AA}$. At face value, however, the incidence rate of weak $\left(W_{\mathrm{r}}<1 \backslash \mathrm{AA}\right)$ systems appears to relatively flat with redshift, while stronger systems appear to become more numerous from $z \sim 6$ to $z \sim 2-3$, before declining towards lower redshifts.

The fact that the evolution in $\mathrm{d} n / \mathrm{d} X$ for strong $\mathrm{Mg}$ II systems shows the same shape as the cosmic star formation rate density has been cited as evidence that these systems are closely linked to star-forming galaxies (Prochter, Prochaska, \& Burles 2006; Ménard et al. 2011; Matejek \& Simcoe 2012). Ménard et al. (2011) also note a correlation between $W_{\mathrm{r}}$ and the [O II] luminosity, a tracer of star formation, enclosed in an SDSS fibre. By comparison, the relatively constant incidence of weak Mg II systems suggests that these absorbers are a feature of metal-enriched haloes that are established early in the process of galaxy formation, and change as a population relatively little, even as other galaxy properties evolve (see discussion in Matejek \& Simcoe 2012). 


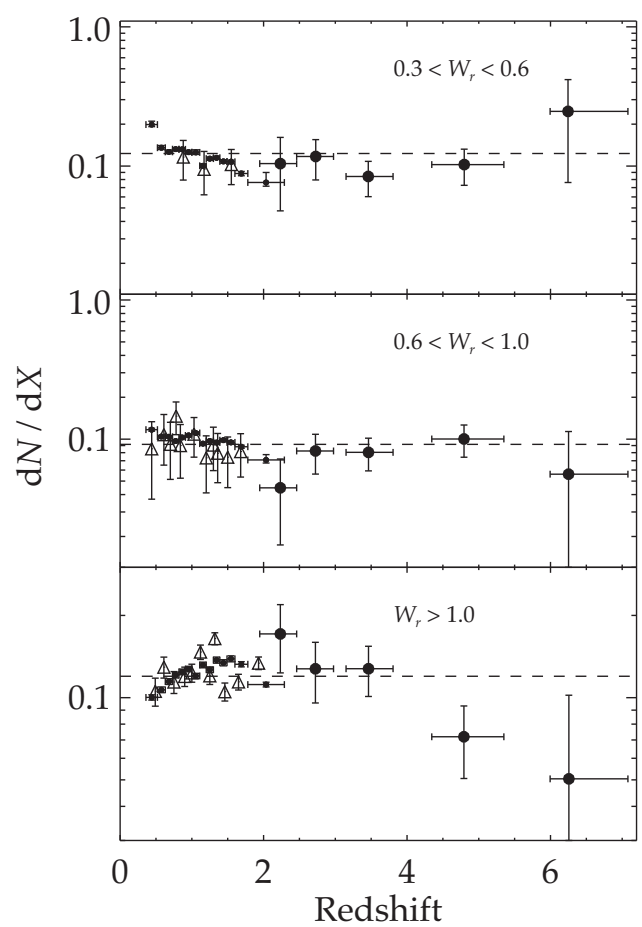

Figure 8. Evolution of the line-of-sight number density of $\mathrm{Mg}$ II absorbers. The three panels give $\mathrm{d} n / \mathrm{d} X$ for three ranges of $\lambda 2796$ rest-frame equivalent width. Triangles are from Nestor, Turnshek, \& Rao (2005). Small circles are from Seyffert et al. (2013). Large circles are from Chen et al. (in preparation). Dashed lines shows the mean $d n / d X$ for the Nestor et al. (2005) and Seyffert et al. (2013) data. For more details, see Chen et al. (in preparation). Figure provided courtesy of R. Simcoe.

\subsubsection{Summary of observations: enrichment versus ionisation}

Although observations of metals near the reionisation epoch are still in an early phase, the results generally indicate substantial metal enrichment in the interstellar and circumgalactic environments of galaxies between $z \sim 6$ and 5. The buildup of metals is expected to correlate with the increase in stellar mass density; however, changes in the ionisation state of the metals must also play a role. For example, the ratio of $N(\mathrm{Si}$ IV $) / N(\mathrm{C}$ IV $)$ in $\mathrm{C}$ IV-selected systems tends to increase with redshift, an indication that higher-redshift systems are tracing denser gas and/or a weaker ionising background (D'Odorico et al. 2013; Boksenberg \& Sargent 2015). The fact that the incidence of low-ionisation systems remains roughly constant out to $z \sim 6$, even while the number density of galaxies is declining with redshift also suggests that the cross-sections of metal-enriched haloes that are largely neutral may be increasing with redshift due to a declining UVB (Becker et al. 2011b; Keating et al. 2014). Matejek et al. (2013) also find evidence that $\mathrm{Mg}$ II systems tend to trace DLAs, which are predominantly neutral, with increasing frequency towards higher redshifts. For high-ionisation systems, shock heating may play a role in setting the ionisation state (Cen \& Chisari 2011), along with the UVB.

\subsection{The metal mass budget}

We now examine in more detail what these observations tell us about high-redshift galaxies and reionisation. First, we ask whether the observed mass density of metals at $z \sim 6$ is consistent with expectations from the star-formation history at higher redshifts. Following Pettini (1999), we can estimate the global mass density of metals by multiplying the stellar mass density by a nucleosynthetic yield derived from stellar models (for similar calculations at lower redshifts, see Peeples et al. 2014; Shull et al. 2014). We emphasise that there are considerable uncertainties in the stellar mass density at these redshifts, the theoretical yields, and, as described above, the measured metal mass densities. This exercise is therefore intended only to give a rough insight into whether the observed metals constitute a reasonable fraction of the metals expected to be produced at $z>6$.

The stellar mass density at $z \sim 6$ for galaxies more massive than $10^{8} \mathrm{M}_{\odot}$ is $\rho_{*} \sim 6 \times 10^{6} \mathrm{M}_{\odot} \mathrm{Mpc}^{-3}$ (González et al. 2011), taking into account the correction factor of 1.6 suggested by Stark et al. (2013) for contamination due to nebular lines. Following Peeples et al. (2014), we adopt a total metal yield of $y=0.030$ for Type II supernovae, which should dominate the metal production at these early times. The yields of oxygen and carbon are taken as $y_{\mathrm{O}}=0.015$ and $y_{\mathrm{C}}=$ 0.0083 , respectively. Using these values, we would expect $\rho_{\mathrm{O}} \sim 9 \times 10^{4} \mathrm{M}_{\odot} \mathrm{Mpc}^{-3}$ and $\rho_{\mathrm{C}} \sim 5 \times 10^{4} \mathrm{M}_{\odot} \mathrm{Mpc}^{-3}$, or $\Omega_{\mathrm{O}} \sim 6 \times 10^{-7}$ and $\Omega_{\mathrm{C}} \sim 4 \times 10^{-7}$. By comparison, the observed mass densities are $\Omega_{\mathrm{OI}} \sim 4 \times 10^{-8}$ (Becker et al. $2011 \mathrm{~b}$ ) and $\Omega_{\mathrm{CII}}+\Omega_{\mathrm{CIV}} \sim 2 \times 10^{-8}$ (Ryan-Weber et al. 2009; Becker et al. 2011b; Simcoe et al. 2011; D’Odorico et al. 2013). The mass density sampled via metal absorption lines is therefore only $\sim 5 \%$ of the expected total. This indicates that the observed metals can easily be produced by the known galaxies at these redshifts. Furthermore, it suggests that a large fraction of the metals reside in phases not easily probed by absorption lines. This includes the metals re-accreted onto stars, in dense pockets of the interstellar medium with small cross-sections, and in ionisation states not directly measured by the available lines. These scenarios are seen in numerical simulations of metal enrichment (e.g. Oppenheimer et al. 2009; Cen \& Chisari 2011; Finlator et al. 2015) Similar conclusions have also been reached at lower redshifts (for a recent, more detailed accounting at $z \sim 0$ see Peeples et al. 2014).

\subsection{Constraints on stellar populations}

Although metal absorption lines provide only a tracer of the total metal budget, their composition can nevertheless provide constraints on the nature of the stars that formed during the reionisation epoch. At $z \gtrsim 5.5$, the Ly $\alpha$ forest becomes too thick to measure $\mathrm{H}$ i column densities of individual absorbers (Section 2.1), which hinders direct metallicity measurements. Relative abundances can still be inferred, however, particularly for low-ionisation systems where ionisation 

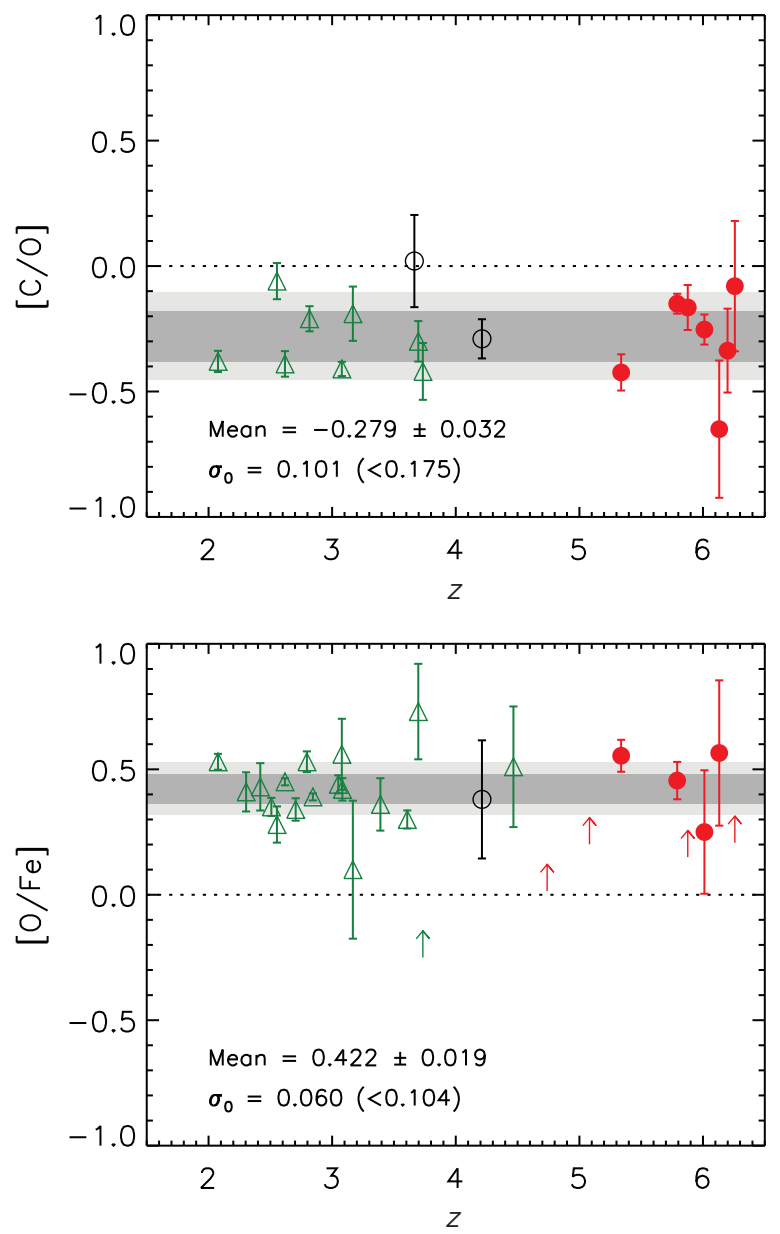

Figure 9. Relative abundances of carbon and oxygen (top panel), and oxygen and iron (bottom panel), expressed logarithmically as a fraction of the solar value. Triangles and arrows at $z<4.5$ are for metal-poor DLAs from Cooke et al. (2011). Open circles are for sub-DLAs from Dessauges-Zavads et al. (2003) and Péroux et al. (2007). Filled circles and arrows at $z>4.7$ are for low-ionisation absorbers from Becker et al. (2012). The mean value given in each panel has been calculated from all measurements. The nominal intrinsic rms scatter (accounting for measurement uncertainties), $\sigma_{0}$, and the 95\% upper limit on $\sigma_{0}$ (in parentheses) are shown as dark and light shaded bands, respectively. Reproduced from Figure 11 of Becker et al. (2012) by permission of the authors and the AAS.

corrections should be minimal (e.g. Wolfe et al. 2005; Becker et al. 2011b). The relative abundances of O, C, Si, and Fe were measured for nine low-ionisation systems at $z \sim 5$ to 6 by Becker et al. (2012). Results for $[\mathrm{C} / \mathrm{O}]$ and $[\mathrm{O} / \mathrm{Fe}]$ are shown in Figure 9, where $[X / Y]$ gives the logarithmic abundances with respect to solar, $[X / Y]=\log (X / Y)-\log (X / Y)_{\odot}$. The results at $z \sim 5-6$ are generally consistent with relative abundances measured in DLAs and sub-DLAs over $2<z<4$ (e.g. Dessauges-Zavads et al. 2003; Wolfe et al. 2005; Péroux et al. 2007; Cooke et al. 2011). Moreover, the absorption line measurements at high redshift are broadly consistent with the abundances in (non carbon-enhanced) metal-poor halo stars (e.g. Cayrel et al. 2004), particularly those with $[\mathrm{O} / \mathrm{H}] \lesssim-2$ (Fabbian et al. 2009) (see discussions in Cooke et al. 2011; Becker et al. 2012). The [O/Fe] values are consistent with enrichment from Type II supernovae (e.g. Chieffi \& Limongi 2004), as expected given there has been little time at $z \sim 5-6$ for a contribution from Type Ia supernovae. The lack of strong variations in the absorption-line ratios suggests that these systems are enriched by broadly similar stellar populations. There is no clear evidence of unusual abundance patterns that would indicate enrichment from exotic sources such as Population III stars, although trends in $[\mathrm{C} / \mathrm{O}]$ among metal-poor DLAs and halo stars may indicate a Population III contribution at the very low-metallicity end $([\mathrm{O} / \mathrm{H}]<-2$; Cooke et al. 2011). In semi-analytic models of galaxy formation including metal enrichment, Kulkarni et al. (2014) also conclude that measured ionic ratios at $z \lesssim 6$, particularly [O/Si], preclude a large contribution from Population III stars to the metal or ionising photon budget during reionisation.

\subsection{The connection to galaxies and reionisation}

Numerous studies over $0<z<5$ have demonstrated that the gas traced by metal lines reflects a cycle of inflows and outflows that help to regulate galaxy growth (e.g. Davé \& Oppenheimer 2007; Steidel et al. 2010; Tumlinson et al.Tumlinson et al. 2011; Turner et al. 2014; Crighton et al. 2015). The lower-redshift studies have shown that galaxies over a wide range of luminosities and star formation rates are surrounded by an enriched circumgalactic medium out to typical distances of at least $\sim 100$ proper kpc (e.g. Simcoe et al. 2006; Bordoloi et al. 2014), indicative of the volume filled by outflows or other enrichment mechanisms. Metal lines at $z \sim 5--6$ should therefore trace the early stages of the enrichment process, helping to elucidate the mechanisms that shape the earliest galaxies. In addition, if metal absorption lines tend to be associated with low-mass galaxies, then these lines may help to identify vital sources of ionising photons that are beyond the reach of current direct galaxy surveys.

We can use lower-redshift observations to gain some insight into the type of galaxies associated with metal absorbers near $z \sim 6$. The line-of-sight number density of $\mathrm{C}$ IV absorbers at $z \sim 6$ with $N_{\mathrm{CIV}}>10^{13} \mathrm{~cm}^{-2}$ is $\mathrm{d} n / \mathrm{d} X \sim 1$ (D'Odorico et al. 2013). For a population with a fixed physical cross-section, $\sigma$, the comoving number density can be computed as

$$
\phi=\frac{\mathrm{d} n}{\mathrm{~d} X} \frac{H_{0}}{c \sigma} .
$$

If we take the lower-redshift value of $100 \mathrm{kpc}$ (proper) as an upper limit on the radius to which haloes are enriched at $z \sim 6$, then the number density of such haloes would be $\phi \sim 7 \times 10^{-3} \mathrm{Mpc}^{-3}$. This corresponds to the number density of galaxies with absolute UV magnitudes $M_{\mathrm{UV}} \lesssim-17$ to -18 (Finkelstein et al. 2015; Bouwens et al. 2015), which is near the limit of direct galaxy surveys. In terms of dark matter haloes, this number density corresponds to 

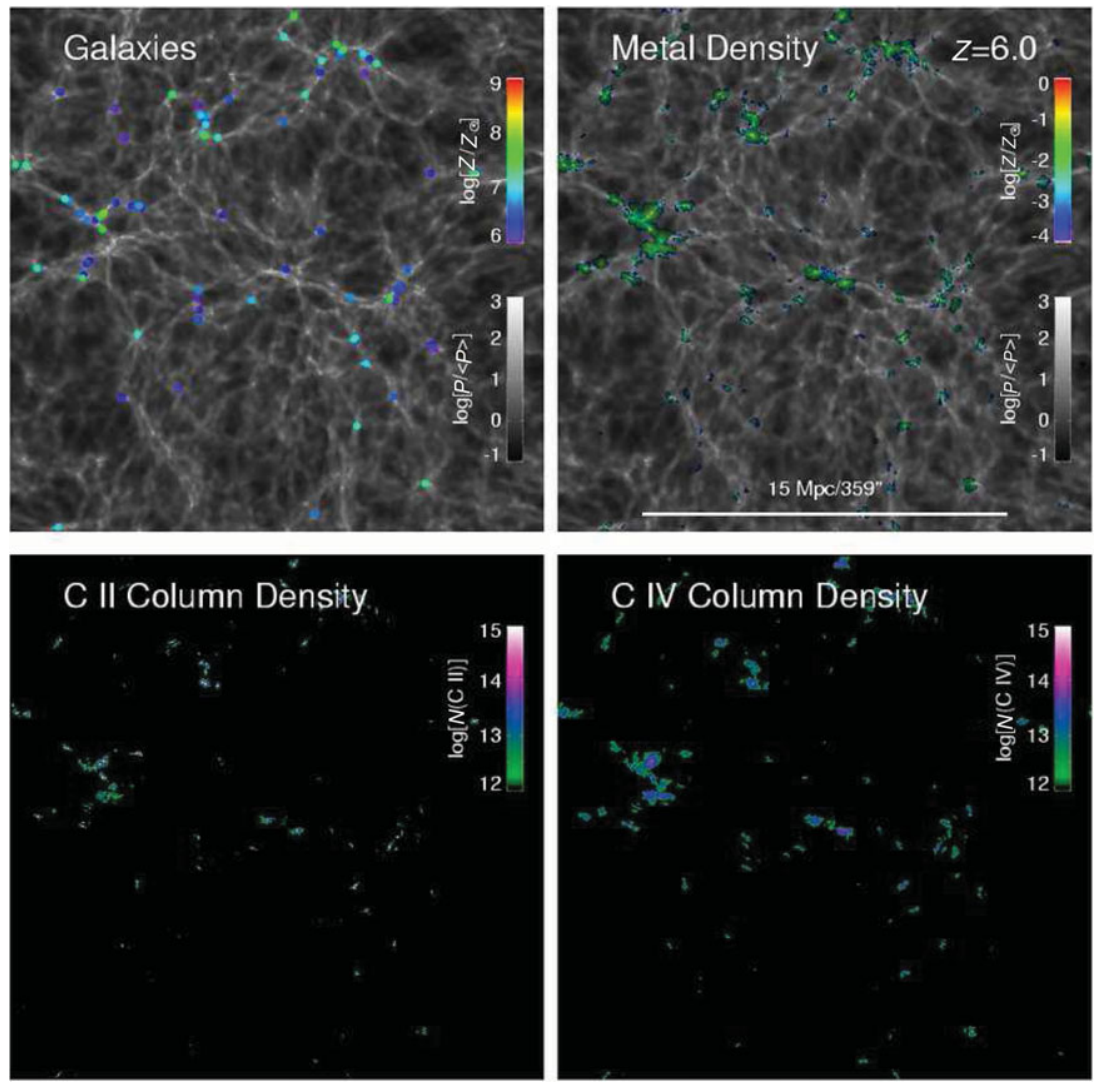

Figure 10. A slice through a hydrodynamical simulation at $z=6.0$ showing the location of galaxies with stellar masses greater than $10^{6} \mathrm{M}_{\odot}$ (upper left), the metallicity as a fraction of solar (upper right), and the integrated C II (lower left) and C IV (lower right) column densities. The grey scale in the upper panels shows the total mass density as a fraction of the mean density. The slice is $16 h^{-1} \mathrm{Mpc}$ on a side and $25 \mathrm{~km} \mathrm{~s}^{-1}$ thick. In this simulation, metal absorbers closely trace the galaxies, with only a small fraction of the volume enriched above $1 / 10,000$ th of solar metallicity. The cross-section of $\mathrm{C}$ IV, which favours lower densities, is more extended than that of C II. Reproduced from Figure 5 of Oppenheimer et al. (2009) by permission of the authors.

haloes with masses $M_{\mathrm{h}} \gtrsim 2 \times 10^{10} \mathrm{M}_{\odot}$ (Murray, Power, \& Robotham 2013). These figures already suggest that metal absorbers at $z \sim 6$ are likely to be associated with relatively modest galaxies, particularly given that an enrichment radius of $100 \mathrm{kpc}$ is probably a conservative upper limit due to the limited time available for metal-enriched outflows to travel by $z \sim 6$ (e.g. Oppenheimer et al. 2009). Further insights can be gained by directly searching for galaxies associated with absorbers, and through more sophisticated modelling.

Directly identifying the galaxies associated with metal absorbers at these redshifts is challenging due to the faintness of the sources and the corresponding difficulty in obtaining spectroscopic redshifts. Díaz et al. (2011, 2014, 2015) have searched for galaxies associated with $\mathrm{C}$ IV systems at $z \simeq 5.7$. In at least one case, on $\sim 10 h^{-1} \mathrm{Mpc}$ scales the C IV absorber appears to be more closely associated with narrow-band-selected Ly $\alpha$ emitting galaxies (LAEs), rather than broad-band-selected LBGs. This suggests that C IV systems may trace lower-density environments dominated by low-mass galaxies (Díaz et al. 2014). Further efforts at identifying galaxies associated with C IV and other types of metal absorbers will help to clarify this picture.

On the theoretical side, considerable efforts have been made to model the production and distribution of metals near reionisation using hydrodynamical simulations (Oppenheimer \& Davé 2006, 2008; Davé \& Oppenheimer 2007; Oppenheimer et al. 2009; Cen \& Chisari 2011; Keating et al. 2014; Pallottini et al. 2014; Finlator et al. 2015) and analytical methods (Bagla, Kulkarni, \& Padmanabhan 2009). The simulations generally support a picture where metals reside in overdense regions, and that only a few percent of the IGM needs to be enriched in order to reproduce the observed line statistics (Figure 10). Metal lines should therefore closely trace the environments of galaxies, and be sensitive to the details of feedback mechanisms (e.g. Oppenheimer \& Davé 2006, 2008; Cen \& Chisari 2011). Finlator et al. (2015) further showed that ion ratios are sensitive to the local variations in the UVB induced by these galaxies (see also Oppenheimer et al. 2009). 
A further conclusion of simulations is that, while absorbers arise from galaxies over a wide range in mass, low-ionisation lines tend to trace lower-mass galaxies than C IV (e.g. Oppenheimer et al. 2009; Finlator et al. 2013). This occurs because strong outflows are required to transport enriched material out to the low densities where C IV becomes a favoured ionisation state. Oppenheimer et al. (2009) find that C IV absorbers with $N_{\mathrm{CIV}} \geq 10^{13} \mathrm{~cm}^{-2}$ should largely be found around galaxies with stellar masses $M_{*}=10^{6.5-8.5} \mathrm{M}_{\odot}(\mathrm{UV}$ magnitudes $M_{\mathrm{UV}} \simeq-14.5$ to -19 ), whereas C II absorbers should trace galaxies that are roughly a factor of 10 less massive. In both cases, the absorber strength is found to anticorrelate with projected distance from the galaxy, and for $\mathrm{C}$ IV it is found to correlate with galaxy stellar mass. The likelihood that metal lines, and in particular low-ionisation lines, trace low-mass galaxies underlines the importance of these lines as probes of the faint galaxies that may be largely responsible for reionisation.

The hydrodynamical simulations discussed above demonstrate that the redshift evolution of essentially all ions is sensitive to (i) the increase in the total mass density of metals towards lower redshifts due to ongoing star formation, (ii) the propagation of enrichment towards lower densities with declining redshift, and (iii) the decline in the mean UV background with redshift at $z>5$ (see also Section 2.3). The last two factors mean that systems traced by C IV (C II) should constitute a decreasing (increasing) fraction of the metals towards higher redshifts (Figure 11). This helps to explain the substantial decline in $\mathrm{C}$ IV from $z \sim 5$ to 6 , even while the number density of low-ionisation absorbers remains relatively constant (Oppenheimer et al. 2009; Keating et al. 2014; Finlator et al. 2015). If these trends continue, then low-ionisation lines may become numerous at $z \gtrsim 7$ (Finlator et al. 2015). Some authors have suggested that a 'forest' of low-ionisation lines such as O I and C II may appear during reionisation if significant amounts of the IGM are enriched at earlier times (Oh 2002; Furlanetto \& Loeb 2003). At present, there is little evidence for an O I forest in the $z \sim 6$ data; however, higher-redshift lines of sight may yet reveal substantial quantities of enriched, neutral gas in the reionisation epoch. The prospects for this depend partly on whether early metal enrichment extends to truly intergalactic regions, or if it is mainly confined to the circumgalactic environments around galaxies.

\section{THE REIONISATION HISTORY}

Thus far, we have examined how quasar absorption line observations elucidate the properties of high-redshift galaxies by probing the post-reionisation UVB and heavy element production in the early Universe. In this section, we now turn to focus on how quasar absorption line studies directly constrain the reionisation history at $z \gtrsim 6$. We begin by briefly describing the basic properties of the EoR relevant for current and future observations of the IGM, and then proceed to examine the current quasar absorption line data.

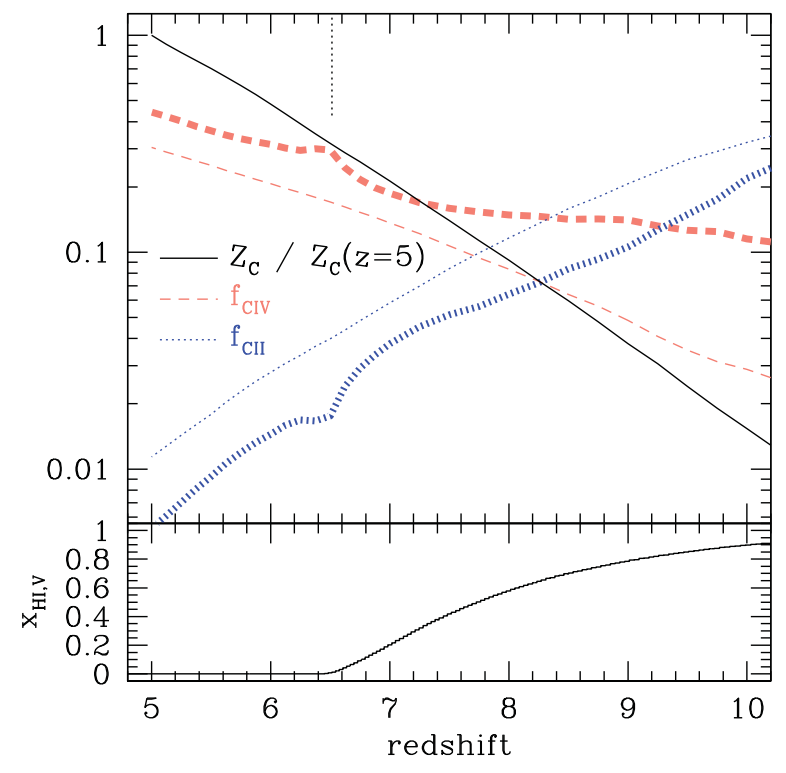

Figure 11. Evolution of the carbon (top panel) and hydrogen (bottom panel) in a radiation hydrodynamical simulation by Finlator et al. (2015). The solid line in the upper panel shows the total metal mass fraction, normalised to its value at $z=5$. The red-dashed and blue-dotted lines show the volumeweighted fractions of carbon that are in C IV and C II, respectively. The thick and thin lines give the results for the UV background predicted by the simulation and for a uniform Haardt \& Madau (2012) background. The solid line in the bottom panel shows the volume-weighted fraction of neutral hydrogen. Simulations similar to this one predict that low-ionisation metal lines should become more prominent relative to high-ionisation lines in the reionisation epoch. Reproduced from Figure 6 of Finlator et al. (2015) by permission of the authors.

\subsection{The IGM during reionisation}

The IGM is expected to resemble a two-phase medium during reionisation, with part of the IGM in highly ionised 'bubbles' that form around collections of galaxies and accreting black holes, while the rest remains mostly neutral (e.g. Figure 12). This two-phase medium can be broadly characterised by two quantities: the volume filling factor of ionised hydrogen and the size distribution of the ionised regions (see e.g Madau et al. 1999; Miralda-Escudé et al. 2000; Gnedin 2000; Furlanetto et al. 2004). A primary goal of reionisation studies is to constrain these properties from observations, and to then use them to inform models of galaxy formation and the highredshift IGM.

However, this two-phase description does not completely describe the ionisation state of the IGM. Firstly, there is also some residual neutral hydrogen within the bubble interiors; this neutral fraction will shrink during and after reionisation as the mean free path of the ionising photons grows; this is eventually observed as the Ly $\alpha$ forest at $z<6$. Secondly, in addition to this highly ionised diffuse gas, there will be some mostly neutral, self-shielded dense clumps (LLSs) that remain, both in the bubble interiors and in the postreionisation IGM (Furlanetto \& Oh 2005; Gnedin \& Fan 2006; Choudhury, Haehnelt, \& Regan 2009; Alvarez \& Abel 

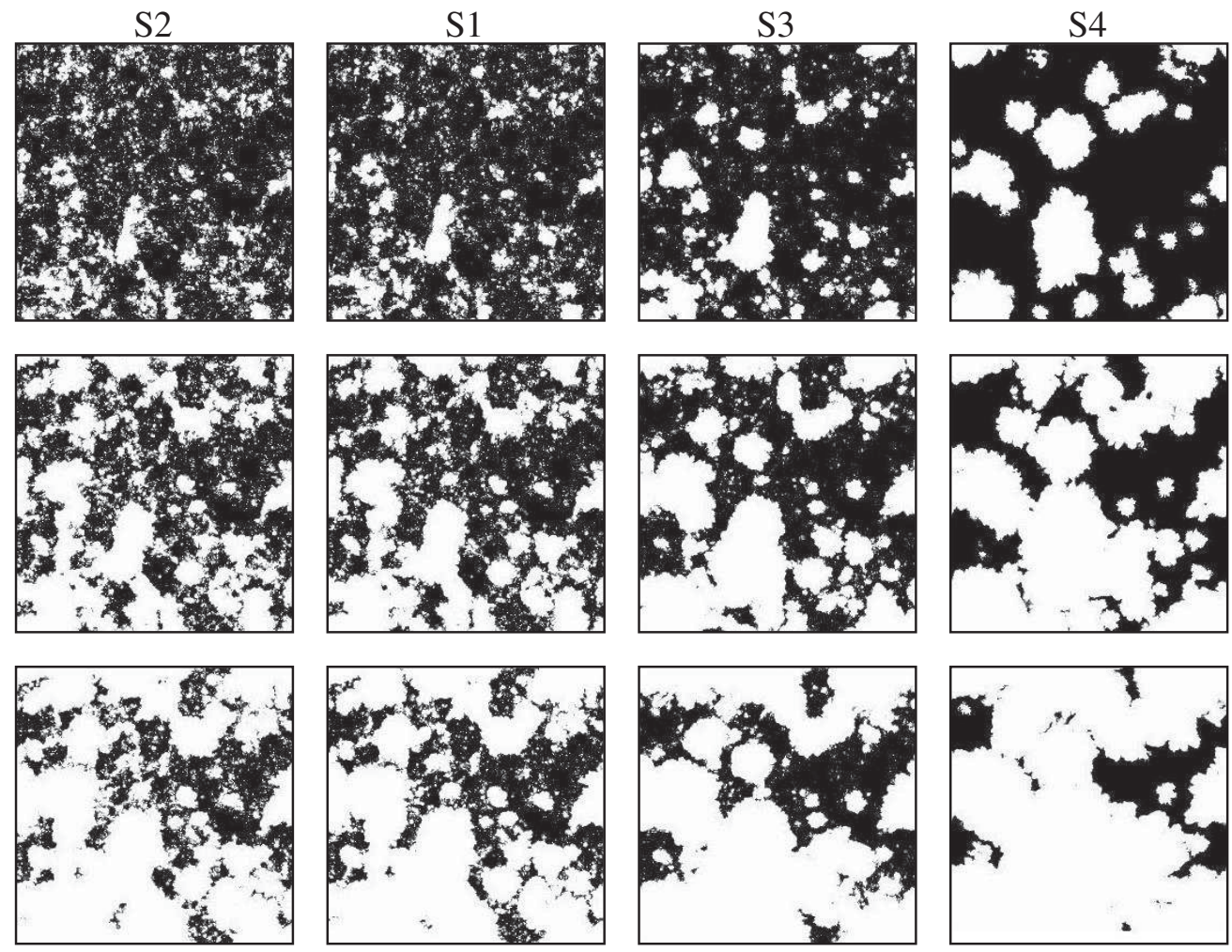

Figure 12. Models illustrating the size and distribution of ionised regions at different stages of reionisation. Each panel shows a slice $\left(0.25 h^{-1}\right.$ comoving Mpc thick) through a numerical simulation of reionisation, spanning $65.6 h^{-1}$ comoving $\mathrm{Mpc}$ on a side. The white regions show highly ionised hydrogen while the dark regions are neutral. The columns, from left to right, show different models for the ionising sources which reside in progressively more massive and highly clustered host haloes. The rows, from top to bottom, show different stages of the reionisation process: in each model the efficiency of the ionising sources is normalised so that the mean ionised fraction is $\left\langle x_{i}\right\rangle=0.2,0.5$, and 0.7, respectively. Reproduced from Figure 3 of McQuinn et al. (2007a) by permission of the authors.

2012; Sobacchi \& Mesinger 2014). These systems play an important role in setting the mean free path for ionising photons. Consequently, at the tail-end of reionisation the IGM can be almost completely filled by ionised gas once bubbles have overlapped, yet the mean free path of ionising photons nevertheless has significant spatial fluctuations (Section 2.7). This intermediate period is what Furlanetto \& Oh (2005) refer to as the transition between the "bubble-dominated and cosmic-web dominated' eras in the reionisation history of the Universe. Capturing this transition era is challenging for modellers, in part because of the large dynamic range in spatial scale involved: this requires fully resolving the dense sinks of ionising photons that (mostly) regulate the mean free path of the ionising photons at the end of reionisation, while simultaneously capturing representative samples of the ionised bubbles and the large-scale spatial variations in the source abundance.

Quasar absorption line measurements play an important role within this context. First, these observations presently provide our most detailed probe of the properties of the highredshift IGM, with current studies extending out to $z \sim 7$ (Fan et al. 2006; Mortlock et al. 2011). In terms of constraining the redshift evolution of the filling factor of ionised regions, these observations may then address whether reionisation completes at $z \leq 7$ or at some earlier time. As we discuss below, there are some interesting hints that reionisation may be incomplete at $z \leq 7$, and there are good prospects for placing more definitive constraints in the future.

\subsection{Mean Ly $\alpha$ forest transmission}

The first measurement to consider is the redshift evolution of the average Ly $\alpha$ forest transmission as a function of redshift, $\langle F\rangle$. As is evident from the Gunn \& Peterson (1965) argument presented in Section 2.1, at the redshifts of interest even gas with neutral fractions of $\left\langle x_{\mathrm{HI}}\right\rangle \gtrsim 10^{-4}-10^{-5}$ should produce highly saturated absorption in the Ly $\alpha$ line. Although this large opacity makes direct inferences about reionisation from the Ly $\alpha$ forest challenging, progress can still be made. Figure 13 displays the observed redshift evolution of the effective optical depth, $\tau_{\text {eff }}^{\alpha}=-\ln \langle F\rangle$, in the Ly $\alpha$ forest at $z>3.8$. Each point shows an estimate from an individual stretch of spectrum of width either $\Delta z \sim 0.15$ or $50 h^{-1}$ comoving Mpc, which are similar at the redshifts of interest. The upward pointing arrows show $1 \sigma$ lower bounds 


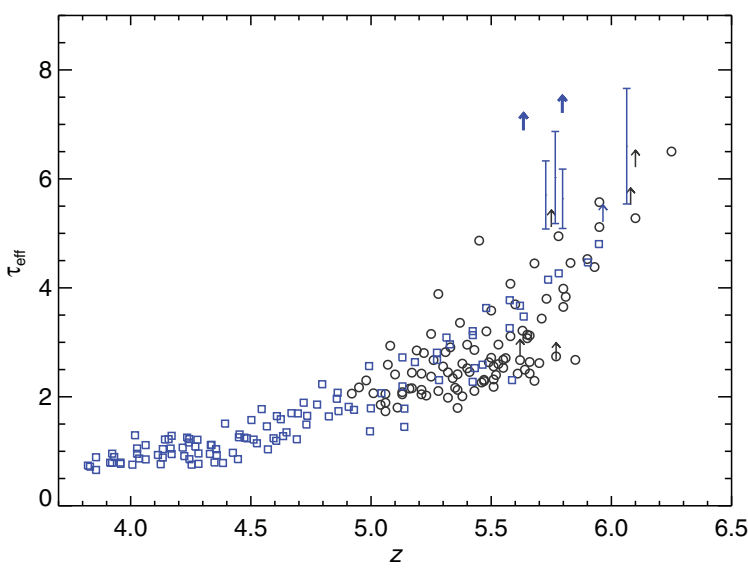

Figure 13. The evolution of the Ly $\alpha$ forest effective optical depth with redshift. The measurements are from Fan et al. (2006) (black circles and arrows, $\Delta z=0.15$ bins) and Becker et al. (2015) (blue circles, error bars and arrows, $\Delta l=50 h^{-1} \mathrm{Mpc}$ bins). The lower limits at $z>5.5$ are obtained where the $\operatorname{Ly} \alpha$ absorption saturates in the presence of a Gunn-Peterson trough. Reproduced from Figure 6 of Becker et al. (2015) by permission of the authors.

on the effective optical depth in regions where the average transmitted flux is consistent with zero.

Figure 13 reveals several striking features. First, the presence of any transmission at $z \leq 6$ might seem to require that reionisation completed by $z=6$. However, this is not completely secure (Lidz et al. 2007; Mesinger 2010). The transmission only demands that some regions in the IGM are highly ionised: since the forest shows a mixture of completely opaque and partly transmissive regions at $z=5--6$ (Fan et al. 2006), it may be that some of the opaque regions are actually significantly neutral, with order unity neutral fractions. It appears feasible that some so-called 'neutral islands' remain as late as $z \sim 5.5$ (Malloy \& Lidz 2015); this possibility is constrained by the dark pixel fraction tests described in Section 4.4.

Additionally, the redshift evolution of $\tau_{\mathrm{eff}}^{\alpha}$ near $z \sim 6$ may be interpreted as being extremely rapid, requiring reionisation to complete near $z \sim 6$ (e.g. Fan et al. 2006; Gnedin $\&$ Fan 2006). However, it remains unclear whether the mean transmission should evolve rapidly as reionisation completes. This behaviour is predicted in numerical simulations if the mean free path, and hence photoionisation rate incident on a typical region, grows rapidly as reionisation finishes (Gnedin 2000; Bauer et al. 2015; Chardin et al. 2015). This may not happen, however, if the mean free path to ionising photons at the end of reionisation is limited by dense photon sinks, and not the size of the ionised regions themselves (Furlanetto \& Mesinger 2009; Alvarez \& Abel 2012).

Finally, the small levels of remaining transmission through the Ly $\alpha$ forest near $z \sim 5-6$ trace only rare, underdense regions of the IGM (Bolton \& Becker 2009). This is the case even if reionisation is complete at these redshifts, and the IGM gas is highly ionised. Extrapolating from the behaviour in low-density voids to make inferences about the overall ionisation state in more typical regions of the IGM can therefore be uncertain and model dependent (Oh \& Furlanetto 2005). In summary, while the mean Ly $\alpha$ forest transmission clearly indicates the IGM neutral fraction is increasing toward $z=6$, on its own it is unable to unambiguously determine the duration and end point of the reionisation process.

\subsection{Higher-order Lyman series lines}

Further progress can be made by considering the transmission through higher-order Lyman-series lines. These transitions have smaller cross-sections ${ }^{10}$ relative to $\mathrm{Ly} \alpha$, and so become fully saturated at larger neutral hydrogen fractions. The analogue of the Gunn-Peterson formula (Equation (2)) for Ly $\beta$ is then simply:

$$
\tau_{\mathrm{GP}}^{\beta}=\frac{\sigma_{\beta}}{\sigma_{\alpha}} \tau_{\mathrm{GP}}^{\alpha}=0.16 \tau_{\mathrm{GP}}^{\alpha} .
$$

It is important to keep in mind, however, that it is the effective optical depths, $\tau_{\text {eff }}=\langle F\rangle$, which are observable, and these do not scale in the same way as the true optical depths (e.g. Songaila \& Cowie 2002; Oh \& Furlanetto 2005). The ratio of $\tau_{\text {eff }, \alpha} / \tau_{\text {eff, } \beta}$ depends on the gas density distribution, the relation between temperature and density (Furlanetto \& Oh 2009), and other aspects of the IGM model. In typical models considered in the literature, $\tau_{\text {eff, } \alpha} / \tau_{\text {eff, } \beta} \sim 3$ (e.g. Fan et al. 2006), which is around a factor of 2 smaller than the ratio of the Ly $\alpha$ and Ly $\beta$ cross-sections. The corresponding factors for higher-order lines, such as Ly $\gamma$, are still larger.

One complication here is that the higher-order transitions land in regions of the spectrum that also contain absorption from lower-order lines, sourced by gas at lower redshift (e.g. Figure 1). For example, a wavelength that contains Ly $\beta$ absorption at $z_{\beta}=6$ also contains Ly $\alpha$ absorption from gas at $z_{\alpha}=\lambda_{\beta}\left(1+z_{\beta}\right) / \lambda_{\alpha}-1=4.9$. However, since these two sources of absorption are widely separated in physical space, they are to a very good approximation uncorrelated (Dijkstra, Lidz, \& Hui 2004b), and so

$$
\left\langle\mathrm{e}^{-\tau} \text { tot }(\lambda)\right\rangle=\left\langle\mathrm{e}^{-\tau} \beta\left(z_{\beta}\right)\right\rangle\left\langle\mathrm{e}^{-\tau_{\alpha}}\left(z_{\alpha}\right)\right\rangle .
$$

The average transmission through the Ly $\alpha$ line at $z_{\alpha}$ can be easily measured using lower-redshift quasar spectra. This expression further generalises to the Ly $\gamma$ region of the quasar spectrum-which contains overlapping absorption from Ly $\gamma$ and lower-redshift absorption in Ly $\beta$ and Ly $\alpha$-and to still higher-order lines.

In practice, measurements of the mean transmission in the Ly $\beta$ and Ly $\gamma$ forest-after dividing out estimates of the foreground absorption in the lower-order lines as described above-are also mostly consistent with saturated absorption

\footnotetext{
${ }^{10}$ For Ly $\beta$, the rest-frame wavelength of the transition is $\lambda_{\mathrm{Ly} \beta}=1025.72 \AA$ and the scattering cross-section is $\sigma_{\mathrm{Ly} \beta}=7.18 \times 10^{-19} \mathrm{~cm}^{2}$. Similarly for $\mathrm{Ly} \gamma, \lambda_{\mathrm{Ly} \gamma}=972.54 \stackrel{\circ}{\mathrm{A}}, \sigma_{\mathrm{Ly} \gamma}=2.50 \times 10^{-19} \mathrm{~cm}^{2}$.
} 
above $z \gtrsim 6$ (Fan et al. 2006). This strengthens the case that the opacity is evolving near $z \sim 6$, and allows a slightly stronger limit on the possibility that the absorption arises from highly ionised gas after reionisation. Nevertheless, even the current upper limits on the transmission through the Ly $\gamma$ forest imply fairly modest lower limits on the neutral fraction of $\left\langle x_{\mathrm{HI}}\right\rangle \gtrsim 10^{-4}--10^{-3}$, depending on the precise model. Hence, even high effective optical depth absorption in Ly $\gamma$ does not, by itself, imply that reionisation is incomplete at $z \gtrsim 6$. However, higher-order Lyman series lines are also useful for constraining the possibility that there are neutral islands left over at $z \leq 6$, since these lines help in placing upper limits on the dark pixel fraction, as described next.

\subsection{Dark pixel fraction and dark gaps}

Recent work has demonstrated that a conservative, almost model-independent lower bound on the filling factor of ionised regions can be placed by counting the fraction of spectral pixels that are completely absorbed (McGreer et al. 2011, 2015). This is because regions with transmission through the $\operatorname{Ly} \alpha$ line are certain to contain highly ionised gas. One can place a stronger limit by including the $\mathrm{Ly} \beta$ region of the quasar spectrum and potentially other higher series lines. In this sense, the dark pixel fraction analysis directly targets one of the main properties of the EoR, the filling factor of ionised bubbles. However, the trade-off is that the limit obtained is conservative and highly ionised regions may be responsible for some (or all) of the dark pixels.

In the first analysis of this sort, McGreer et al. (2011) found that the volume-averaged neutral fraction needs to be smaller than $\left\langle x_{\mathrm{HI}}\right\rangle<0.2$ at $5 \leq z \leq 5.5$, and smaller than $\left\langle x_{\mathrm{HI}}\right\rangle<0.5$ at $z=6$, each at $1 \sigma$ confidence. A subsequent study incorporating new, higher signal to noise spectra has improved these limits to $\left\langle x_{\mathrm{HI}}\right\rangle<0.11$ at $z=5.6,\left\langle x_{\mathrm{HI}}\right\rangle<$ 0.09 at $z=5.9$ at $1 \sigma$ confidence (McGreer et al. 2015). At $z=6.1$, there are significantly more dark pixels and the limit is weaker with $\left\langle x_{\mathrm{HI}}\right\rangle<0.58$, again at $1 \sigma$ confidence. These results suggest that if significantly neutral diffuse gas remains at $z \leq 6$, it fills a rather small fraction of the IGM volume.

An approach to search for direct signatures of any remaining neutral islands is to stack spectra around long, highly absorbed regions in the Ly $\alpha$ and Ly $\beta$ forests (Malloy \& Lidz 2015). If significantly neutral gas remains in some of the fully absorbed regions, the stacked spectra should recover slowly as one moves from absorbed to transmitted regions, owing to the damping wing of the Ly $\alpha$ line from diffuse neutral gas in the IGM (Miralda-Escudé 1998; Malloy \& Lidz 2015). However, one can only apply this test at redshifts where the spectra show both completely absorbed regions and regions with some transmission. In typical regions of the IGM, this means this test can only be applied at $z \lesssim 6$.

Finally, a related diagnostic is the size distribution of contiguous saturated regions in the Ly $\alpha$ forest, and the redshift evolution of these so-called 'dark gaps' (e.g. Songaila \& Cowie 2002; Paschos \& Norman 2005; Fan et al. 2006;

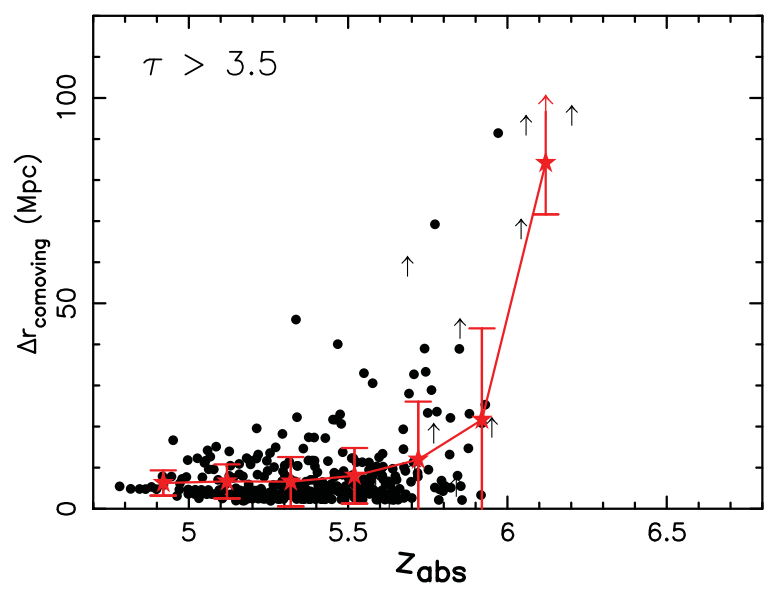

Figure 14. The size of dark gaps as a function of redshift measured from 12 quasar spectra obtained with Keck/Echellete Spectrograph and Imager. The gaps are defined as contiguous regions in the observed Ly $\alpha$ forest where the optical depth never goes beneath the threshold of $\tau_{\alpha}<3.5$. The upward pointing arrows show lower limits on the dark gap size for observed gaps that are close to the quasar near-zone (see Section 4.5). The spectra at $z \gtrsim 5.2$ show fully absorbed regions that are tens of comoving Mpc in size, and the dark gap sizes grow steeply in length at $z \gtrsim 5.7$. Reproduced from Figure 10 of Fan et al. (2006) by permission of the authors and the AAS.

Gallerani et al. 2006, 2008; Mesinger 2010). Some measurements of dark gap sizes and their redshift evolution are shown in Figure 14. As with many of the other observed properties of the $z \sim 6$ Ly $\alpha$ forest, the abundance of large dark gaps grows steeply near $z \sim 6$. On the other hand, even by $z \sim 5.3$, there are some $\gtrsim 30 \mathrm{Mpc}$ contiguous regions that appear entirely absorbed, with $\tau_{\alpha} \geq 3.5$ across the entire stretch of spectrum. This evolution might result in part from the presence of remaining neutral islands in the IGM, or may instead reflect the thickening of the Ly $\alpha$ forest owing to the increasing mean density of the Universe and the dropping intensity of the UVB, along with fluctuations in the mean free path and the IGM temperature. As with many of the other $z \sim 6$ measurements, improved models of the transition era-in which the IGM is filled with ionised gas, yet the mean free path still has sizable spatial fluctuations-will help with unravelling the precise implications of the dark gap distributions and their redshift evolution.

\subsection{Quasar near-zones}

As already discussed, at $z \gtrsim 6$ the Ly $\alpha$ forest is almost completely saturated. Inferring the ionisation state (and other properties) of the $z \gtrsim 6$ IGM from typical regions of the Ly $\alpha$ forest is therefore challenging. However, gas in spectral regions close to the background quasar itself, in the proximity or 'near-zone', are exposed to ionising radiation from the nearby quasar as well as the UVB. These regions show some transmission through the Ly $\alpha$ forest, and can hence be used to study the $z \gtrsim 6$ IGM.

The simplest measurable property is the size of these zones. Observationally, this is usually defined as the 
distance over which the continuum normalised transmission first drops below some threshold; in Fan et al. (2006) the threshold was chosen to be $F=0.1$, after smoothing each

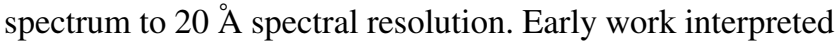
the sizes of these zones as indicating the radii of ionised bubbles expanding around the quasars into a largely neutral IGM (Wyithe \& Loeb 2004; Wyithe, Loeb, \& Carilli 2005). However, subsequent work showed that the transmission profile may instead reflect only the classical proximity effect: the transmission will fall below the chosen threshold in a highly ionised IGM when the combined photoionisation rate from the quasar and background galaxies is small enough (Bolton \& Haehnelt 2007a, 2007b; Maselli et al. 2007, 2009; Lidz et al. 2007). This means that the observed size is an unreliable indicator of the position of the ionisation front around the quasar; this is essentially the usual problem that the Ly $\alpha$ optical depth is large even for highly ionised gas at $z \sim 6$ (see Equation 3).

Nevertheless, the size of the near-zones does evolve with redshift near $z \sim 6$ (Fan et al. 2006). Carilli et al. (2010) fit a linear function to the near-zone size as a function of redshift from $z=5.7$ to $z=6.4$, and find that the proper size-after correcting for differences in quasar luminosities-drops by over a factor of 2 across this narrow redshift interval. This evolution may be driven by rapid evolution in the ionising photon mean free path just after reionisation completes (Wyithe, Bolton, \& Haehnelt 2008). However, the size evolution may also partly reflect the presence of remaining diffuse neutral gas in the IGM, and so the interpretation of the observed evolution is still unclear.

In another approach, Mesinger \& Haiman (2004), Mesinger \& Haiman (2007), and Schroeder, Mesinger, \& Haiman (2013) have compared the near-zone transmission in both Ly $\alpha$ and Ly $\beta$ to mock spectra. The latter study compares mock spectra with three quasar spectra at $z=6.2,6.3$, and 6.4 and argues that each spectrum prefers the presence of damping wing absorption blueward of the Ly $\alpha$ line, arising from the natural broadening of the transition. Crucially, this damping wing is prominent only if the gas is significantly neutral (Miralda-Escudé 1998). A potential source of confusion, however, is from high column density absorbersDLAs - which also have prominent damping wings. However, the damping wing from a DLA has a different shape compared to extended, diffuse neutral gas in the IGM, so these two possibilities may be distinguished, at least in principle (Miralda-Escudé 1998).

In practice, however, it can be difficult to distinguish damping wings from possible diffuse neutral gas and DLAs over a limited stretch of spectrum. In addition, multiple neutral regions may each contribute through their damping wings to the absorption in a given pixel and impact the form of the damping wing profile from the diffuse IGM (Mesinger \& Furlanetto 2008a; McQuinn et al. 2008; Malloy \& Lidz 2015). Based on the spectra alone, Schroeder et al. (2013) cannot rule out the possibility that the putative damping wing comes from a DLA. However, DLAs of the required column

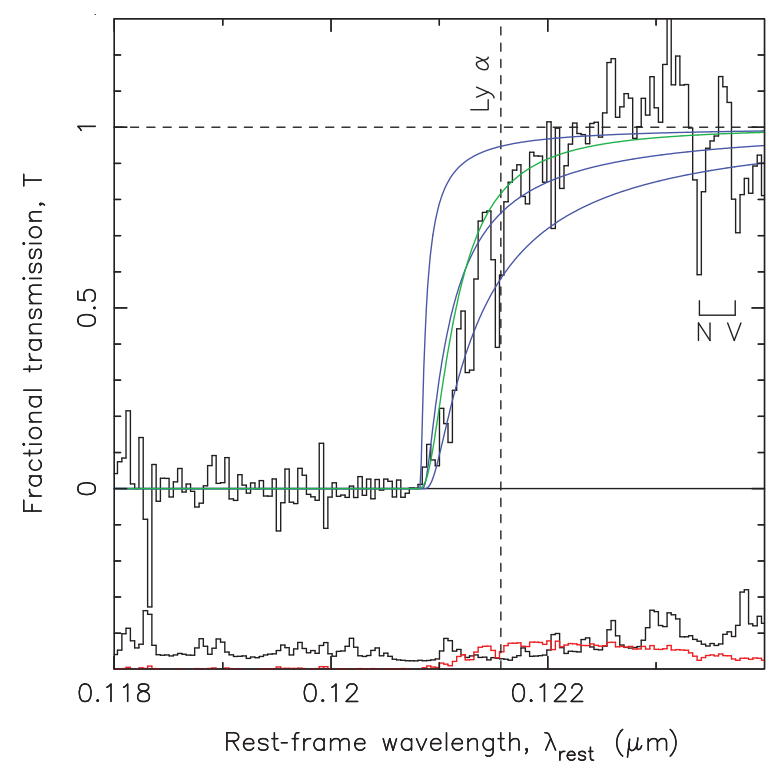

Figure 15. Possible damping wing feature in the spectrum of a $z=7.1$ quasar. The black binned data shows an estimate of the transmission near the systemic redshift (dashed line) of the quasar ULAS J1120+0641. The blue lines show damping wing absorption models (assuming a fully transparent ionised zone around the quasar for simplicity), and uniform neutral fractions of $\left\langle x_{\mathrm{HI}}\right\rangle=0.1,0.5$, and 1.0, with the more neutral models giving more absorption. The ionised zone is assumed to end abruptly, 2.2 Mpc in front of the quasar. The green solid line shows an alternate fit in which the IGM is highly ionised and the damping wing is instead sourced by a DLA of column density $N_{\mathrm{HI}}=4 \times 10^{20} \mathrm{~cm}^{-2}$, situated $2.6 \mathrm{Mpc}$ in front of the quasar. Reproduced from Figure 4 of Mortlock et al. (2011) by permission of the authors and Macmillan Publishers Ltd.

density should be rare and these authors find that the data prefer incomplete reionisation at $z \sim 6$ and a neutral fraction of $\left\langle x_{\mathrm{HI}}\right\rangle \geq 0.1$ at $95 \%$ confidence.

Perhaps, the most intriguing near-zone result comes from the spectrum of the highest-redshift quasar presently known, at $z=7.1$, which shows possible evidence for damping wing absorption on the red side of its Ly $\alpha$ line (Mortlock et al. 2011). The spectrum close to the Ly $\alpha$ emission line, along with toy damping wing models for a partly neutral IGM and a DLA, are shown in Figure 15. This comparison suggests that the damping wing could be sourced by significantly neutral material, although the fully neutral model produces too strong a wing. Bolton et al. (2011) performed a detailed comparison of the transmission profile with simulated spectra, and find that either a neutral fraction of $\left\langle x_{\mathrm{HI}}\right\rangle \gtrsim 0.1$ is required, or a highly ionised IGM can be reconciled with the data if a DLA lies within $\sim 5$ proper Mpc of the quasar (see also Keating et al. 2015). There are two challenges for the DLA scenario however. First, a DLA of the required column density should be rare: in their model, Bolton et al. (2011) find a $5 \%$ probability for a DLA of the requisite properties. A second argument against a DLA is that there is no detectable metal line absorption at the same redshift as the damping wing feature (Simcoe et al. 2012). Quantitatively, this study derives an upper limit on the metal abundance of $\leq 10^{-4}$ 
times the solar abundance for a dense absorber, although the feature could nevertheless be due to a very low-metallicity proto-galaxy.

An alternative use of these proximity or near-zones is measuring the temperature of the IGM at $z \simeq 6$ (Bolton et al. 2010, 2012; Padmanabhan, Choudhury, \& Srianand 2014); the long cooling time for the low-density gas in the IGM allows the gas temperature to be used as a probe of the timing of reionisation (Miralda-Escudé \& Rees 1994; Theuns et al. 2002; Hui \& Haiman 2003). High-redshift temperature measurements close to the EoR are therefore desirable; this limits the time available for gas to cool following reionisation and also bypasses the expected heating from He IIreionisation at $z<4$. In an analysis of gas temperatures inferred from the line widths in near-zones observed in seven high-resolution ( $R \sim 40000)$ quasar spectra at $5.8<z<6.4$, Raskutti et al. (2012) concluded these data were consistent with reionisation completing at $z>6.5$ at $95 \%$ confidence. However, this inference is model dependent, and relies on assumptions for the typical spectrum of ionising sources during reionisation as well as the amount of photoheating by the quasar itself.

Finally, note that an important systematic concern in all these analyses is fitting the unabsorbed quasar continuum close to the Ly $\alpha$ line. Simcoe et al. (2012) investigate this issue, considering four different composite spectra derived from various lower-redshift observations, and also perform a principle component analysis in an effort to extrapolate the continuum estimate from the red side of $\operatorname{Ly} \alpha$. They find that the damping wing fits are fairly stable across the range of continuum fits considered. Nevertheless, it may be helpful to further explore the range of possible continuum shapes close to Ly $\alpha$ in lower-redshift spectra; these spectra can serve as a control sample from an epoch when the IGM is certainly ionised (Kramer \& Haiman 2009; Bosman \& Becker 2015).

\subsection{Consistency with other probes of reionisation}

Having summarised the implications of current quasar absorption line observations for our understanding of the EoR, we now consider these studies in the context of a wide range of complementary multi-wavelength EoR observations. We start with a brief description of existing reionisation probes, and then turn to consider current constraints from these observations.

- CMB: As CMB photons propagate from the surface of last scattering, they may scatter off the free electrons that are (again) prevalent during and after reionisation. This has the effect of damping the primary temperature anisotropies and generating large-scale polarisation anisotropies (Zaldarriaga 1997; Hu \& White 1997), while also producing secondary anisotropies through the kinetic Sunyaev-Zel'dovich (kSZ) effect (Gruzinov \& Hu 1998; Zahn et al. 2005; McQuinn et al. 2005). The distinctive large-scale polarisation signal depends on the total probability that a CMB photon Thomson scatters off a free electron along the line of sight. This is quantified by the electron scattering optical depth, $\tau_{\mathrm{e}}$; this measurement therefore constrains an integral over the entire reionisation history. The patchy $\mathrm{kSZ}$ effect results when $\mathrm{CMB}$ photons scatter off free electrons in ionised regions during the EoR and receive a redshift or blueshift owing to the peculiar velocity of these regions. The small-scale CMB fluctuations that are induced arise in part because of spatial variations in the ionisation field (Aghanim et al. 1996; Gruzinov \& Hu 1998).

- UV luminosity functions: Measurements using the Wide Field Camera 3 (WFC3) on board the Hubble Space Telescope (HST) have found large populations of high-redshift galaxy candidates-some of which are spectroscopically confirmed-using the Lyman break technique (see also Section 2.6). Ground-based surveys have also played an important role at the bright end of the luminosity function (Bowler et al. 2014). These observations have allowed measurements of the UV luminosity function of LBGs at rest-frame wavelength of $1500 \stackrel{\circ}{\mathrm{A}}$ out to $z \sim 8$ (Oesch et al. 2013; McLure et al. 2013; Finkelstein et al. 2015; Ishigaki et al. 2015). There are also now a handful of candidates out to $z \sim 9-10$ (Bouwens et al. 2015; McLeod et al. 2015). These measurements allow the contribution of observed galaxies to the overall ionising photon budget to be assessed (Duncan \& Conselice 2015; Robertson et al. 2015; Bouwens et al. 2015), modulo uncertainties in the escape fraction, the galaxy UV spectral energy distributions, and the contribution from faint, unobserved sources (see discussion in Section 2.6).

- Lyman $\alpha$ emitter surveys: Another highly successful approach for finding high-redshift galaxies uses a narrow-band selection technique to target objects with prominent Ly $\alpha$ emission lines (see Dijkstra 2014 and references therein). The visibility of these Ly $\alpha$ emitters (LAEs) is impacted by the damping wing arising from neutral gas in the IGM (Miralda-Escudé 1998), and so these surveys are sensitive to the reionisation history. Consequently, the abundance of observable LAEs will drop and their clustering will increase as one probes deeper into the EoR (Furlanetto et al. 2006b; McQuinn et al. 2007b; Mesinger \& Furlanetto 2008b).

- GRB optical afterglows: The optical afterglow spectra of GRBs are potentially useful in searching for the damping wing redward of the Ly $\alpha$ emission line (Totani et al. 2006; Totani et al. 2014; Chornock et al. 2013). These sources are extremely luminous (for a short time) and so can be detected at high redshift. Compared to quasars, GRB optical afterglows also have a simpler unabsorbed continuum spectrum and are more likely to occur in typical ionised regions (which are smaller than the ionised bubbles around quasars). On the other hand, GRB afterglow spectra typically show 
high-column density DLA absorption from neutral hydrogen in the host galaxy. The absorption from the GRB host can overwhelm any damping wing signature from diffuse neutral gas in the IGM. However, an afterglow spectrum without prominent host absorption will allow a detection of - or sharper upper limits on the presence of-diffuse neutral gas in the IGM (McQuinn et al. 2008).

- Redshifted 21-cm line: Perhaps, the most promising way of studying reionisation is to detect redshifted 21$\mathrm{cm}$ emission from neutral gas in the IGM during reionisation (Madau et al. 1997; Furlanetto et al. 2006a). Firstgeneration surveys aiming to detect this signal have started to place upper limits on the amplitude of redshifted 21-cm fluctuations (Paciga et al. 2013; Parsons et al. 2014; Dillon et al. 2014). These limits do not yet place interesting constraints on the ionisation history, however, and are therefore not discussed further here. We nevertheless mention this probe in passing, since it may ultimately provide the most powerful approach for studying reionisation.

A selection of current constraints on reionisation are summarised in Figure 16, from Robertson et al. (2015). We first consider constraints on $\tau_{\mathrm{e}}$ from the large-scale polarisation CMB power spectrum measurements. Wilkinson Microwave Anisostropy Probe (WMAP) nine-year E-mode polarisation data, combined with Planck temperature anisotropy data, give $\tau_{\mathrm{e}}=0.089_{-0.014}^{+0.012}(1 \sigma$ error bars, Planck Collaboration et al. 2014; Bennett et al. 2013); this optical depth corresponds to an (instantaneous) reionisation redshift of $z_{\mathrm{r}}=$ $11.1 \pm 1.1$. Recent large-scale polarisation data from the 2015 Planck data release, however, suggest a smaller optical depth of $\tau_{\mathrm{e}}=0.066 \pm 0.016$, when Planck polarisation data are combined with temperature anisotropy and CMB lensing measurements from Planck (Planck Collaboration et al. 2015). The optical depth ${ }^{11}$ preferred by Planck corresponds to an instantaneous reionisation redshift of $z_{\mathrm{r}}=8.8_{-1.4}^{+1.7}$. In practice, this measurement translates into an end point for reionisation which may be close to $z \simeq 6$.

Second, we consider the patchy kSZ effect. Studies using the South Pole Telescope (SPT) placed upper limits on the amplitude of CMB fluctuations induced by the patchy kSZ effect at multi-pole moments of $\ell \sim 3,000$ (Zahn et al. 2012; George et al. 2015, see also Mesinger et al. 2012). A grid of patchy ksZ effect models have been used to place an upper limit on the duration of reionisation of $\Delta z \leq 7.9$ at $95 \%$ confidence (Zahn et al. 2012). The duration here is defined as

\footnotetext{
${ }^{11}$ Note this corresponds to the 'TT+lowP+lensing' constraint in Table 4 reported by Planck Collaboration et al. (2015). The optical depth varies somewhat with the precise data included, e.g. the 'TT+lowP' constraint from only temperature and polarisation anisotropy data (i.e. without CMB lensing) gives $\tau_{\mathrm{e}}=0.078 \pm 0.019$. However, all combinations of the recent Planck measurements prefer a lower optical depth than the WMAP nine-year results quoted above. This is mostly driven by improvements in cleaning polarised foreground emission; these improvements are enabled by the new Planck 353-GHz polarisation maps.
}

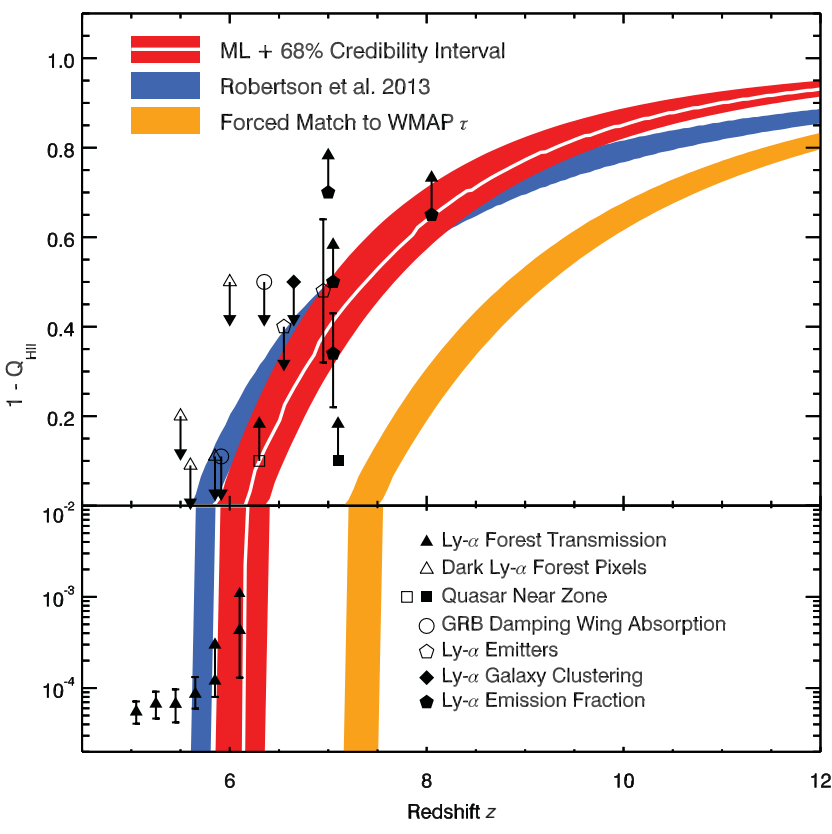

Figure 16. A summary of current multi-probe reionisation constraints. The filling factor of neutral hydrogen is displayed as a function of redshift. The constraints shown in the legend are from the Ly $\alpha$ forest transmission (Fan et al. 2006), dark Ly $\alpha$ forest pixels (McGreer et al. 2015), quasar near-zones (Mortlock et al. 2011; Bolton et al. 2011; Schroeder et al. 2013), GRB damping wing absorption (Chornock et al. 2013), Ly $\alpha$ emitters (Ota et al. 2008; Ouchi et al. 2010), Ly $\alpha$ galaxy clustering (McQuinn et al. 2007b; Ouchi et al. 2010) and the Ly $\alpha$ emission fraction (Schenker et al. 2014; Pentericci et al. 2014). The white line and red band show a reionisation history consistent with the observed star formation rate density and the Planck electron scattering optical depth (Planck Collaboration et al. 2015). The orange band shows a model consistent with the earlier WMAP-9 constraint. Reproduced from Figure 3 of Robertson et al. (2015) by permission of the authors and the AAS.

the redshift interval over which the ionisation fraction transitions from $\left\langle x_{i}\right\rangle=0.20$ to $\left\langle x_{i}\right\rangle=0.99$. Note, however, this limit is dependent on separation from other contributions to the anisotropies, such as the thermal SZ (tSZ) effect and the Cosmic Infrared Background (CIB), using their differing frequency and angular scale dependence. Here, we have quoted the most conservative constraints that allow a large (anti)correlation between the tSZ and CIB: in the absence of any correlation, the $95 \%$ confidence limit shrinks to $\Delta z \leq 4.4$. A more recent analysis by George et al. (2015) finds a similar value of $\Delta z \leq 5.4$ at $95 \%$ confidence.

The implications of the UV luminosity functions of LBGs for reionisation are discussed in Section 2.6. Here, we simply remark that although the details depend on the assumed escape fraction, ionising spectrum, and faint-end extrapolation, these observations are compatible with reionisation completing late, at $z \leq 6$ or so. The red band in Figure 16 displays a reionisation model consistent with UV luminosity functions extrapolated to absolute magnitudes of $M_{\mathrm{UV}} \simeq-13$ and a constant escape fraction $f_{\text {esc }}=0.2$ (Robertson et al. 2015). This is also in accord with the recent Planck optical depth measurements (Planck Collaboration et al. 2015). 
Results from a selection of LAE surveys are also shown in Figure 16. Recent studies of the abundance of LAEs detect a relatively small drop from $z=5.7$ to $z=6.6$, while these surveys are thus far finding very few galaxies at $z=7.3 .^{12}$ Based on the lack of LAE candidates in the Subaru Deep Field at $z=7.3$, Konno et al. (2014) argue that the neutral filling factor is $\left\langle x_{\mathrm{HI}}\right\rangle=0.3-0.8$ at $z=7.3$. From the $z=6.6$ LAE abundance, Ouchi et al. (2010) place an upper limit of $\left\langle x_{\mathrm{HI}}\right\rangle \leq 0.4$, and from the lack of strong LAE clustering these authors conclude that $\left\langle x_{\mathrm{HI}}\right\rangle \leq 0.5$. Finally, from measuring the redshift evolution of the Ly- $\alpha$ fraction-the fraction of LBGs that emit appreciably in Ly $\alpha$-Schenker et al. (2014) find $\left\langle x_{\mathrm{HI}}\right\rangle=0.34_{-0.12}^{+0.09}$ at $z=7$ and $\left\langle x_{\mathrm{HI}}\right\rangle>0.65$ at $z=8$ $(1 \sigma)$. Note, however, these inferences are model dependent; see e.g. Bolton \& Haehnelt (2013), Dijkstra et al. (2014), Taylor \& Lidz (2014), Pentericci et al. (2014), Choudhury et al. (2015), and Mesinger et al. (2015) for further discussion regarding these results.

Finally, current high-redshift GRB optical afterglow spectra have mostly been limited by the presence of strong host DLA absorption, as we alluded to previously. Nevertheless, some limits are possible. For example, Totani et al. (2006) find $\left\langle x_{\mathrm{HI}}\right\rangle<0.60$ at $95 \%$ confidence at $z=6.3$, while Chornock et al. (2013) find a $2 \sigma$ upper limit of $\left\langle x_{\mathrm{HI}}\right\rangle<0.11$ at $z=5.91$.

In summary, when compared to the constraints from quasar absorption lines discussed earlier in this section and displayed in Figure 16 (Fan et al. 2006; Bolton et al. 2011; Mortlock et al. 2011; Schroeder et al. 2013; McGreer et al. 2015), at present the various probes appear broadly consistent with each other. The current data point to a scenario where the completion redshift (when the filling factor of ionised hydrogen reaches unity) is relatively late, between $5.5 \leq z \leq 7$. However, as discussed there remain many significant uncertainties associated with all these measurements-the challenge is therefore to make a more precise and definitive statement. As we now briefly discuss, in the next decade the prospects for further progress toward this goal are promising.

\section{CONCLUSIONS AND FUTURE PROSPECTS}

Quasar absorption line studies yield a rich body of information describing the ionisation, thermal, and chemical enrichment history of the intergalactic gas, allowing us to study the evolution of most of the baryons in the Universe all the way out to-and perhaps into- the EoR. The properties of the intergalactic gas are in turn determined by the underlying cosmic web of structure formation-as specified, for example, according to the currently favoured inflationary, cold dark matter cosmological model with dark energy_along with the properties of galaxies and AGN, which strongly influence the gas in their surroundings. The combination of IGM

\footnotetext{
${ }^{12}$ These redshifts $(z=5.7,6.6,7.3)$ correspond to narrow gaps between bright night sky lines, where LAEs can be found efficiently from the ground using the narrow-band technique.
}

measurements with direct censuses of galaxies and AGN then provide a powerful probe of early galaxy and structure formation.

In the next 5-10 years, there are excellent prospects for improving our understanding of the EoR using quasar absorption lines. First, we emphasise that several independent observations, summarised in Figure 16, are now converging towards reionisation histories that complete sometime between $5.5 \leq z \leq 7$; these are redshifts at which significant amounts of quasar absorption line data already exist. This further motivates studies aimed at understanding reionisation's precise signatures - and that of reionisation's immediate aftermath - in absorption line spectra. Renewed efforts to model the challenging end phases of the EoR, in conjunction with novel data analysis techniques, may therefore lead to breakthroughs in our understanding of the EoR using only currently available data.

Still more compelling, though, will be the advances enabled by future data sets. Current optical and nearinfrared wide-area photometric surveys such as those from Panoramic Survey Telescope and Rapid Response System (Pan-STARRS), the Dark Energy Survey (DES), and the Visible and Infrared Survey Telescope for Astronomy (VISTA) are already increasing the number of known quasars at $z \sim 6--7$ (e.g. Venemans et al. 2013, 2015; Bañados et al. 2014; Reed et al. 2015). Over the next several years, these surveys should roughly double the number of known quasars at $z \sim 6$ and provide the first significant samples at $z \geq 7$. These quasars will provide greatly improved statistics for metal line, Ly $\alpha$ forest, and quasar near-zones studies at $z \geq 6$. For example, they should reveal whether the possible red damping wing feature observed by Mortlock et al. (2011) at $z \sim 7$ is ubiquitous amongst quasars near this redshift, and may uncover evidence of chemical enrichment from metal-free stars. Further dramatic increases in the number of known high-redshift quasars will come from deep surveys conducted by the Large Synoptic Survey Telescope (LSST), Euclid, and the Wide-Field Infrared Survey Telescope (WFIRST).

In the next decade, there will be further progress from high-resolution spectrographs on 30 metre class telescopes, such as the Thirty Meter Telescope (TMT), the Giant Magellan Telescope (GMT), and the European Extremely Large Telescope (E-ELT). The enhanced collecting area of these telescopes will allow significant improvements in the spectroscopy of high-redshift quasars. First, it will enable spectroscopic follow-up of much fainter quasars than is currently feasible in reasonable integration times. Since the quasar luminosity function is quite steep, this translates into a significant boost in the density of quasar sightlines that may be probed spectroscopically, as illustrated in Figure 17. This will improve statistics, and especially provide large samples of close quasar pairs at high spectral resolution; this can be used to measure, among other quantities, the pressure or Jeans smoothing scale, which is sensitive to the timing of reionisation (e.g. Rorai, Hennawi, \& White 2013). 

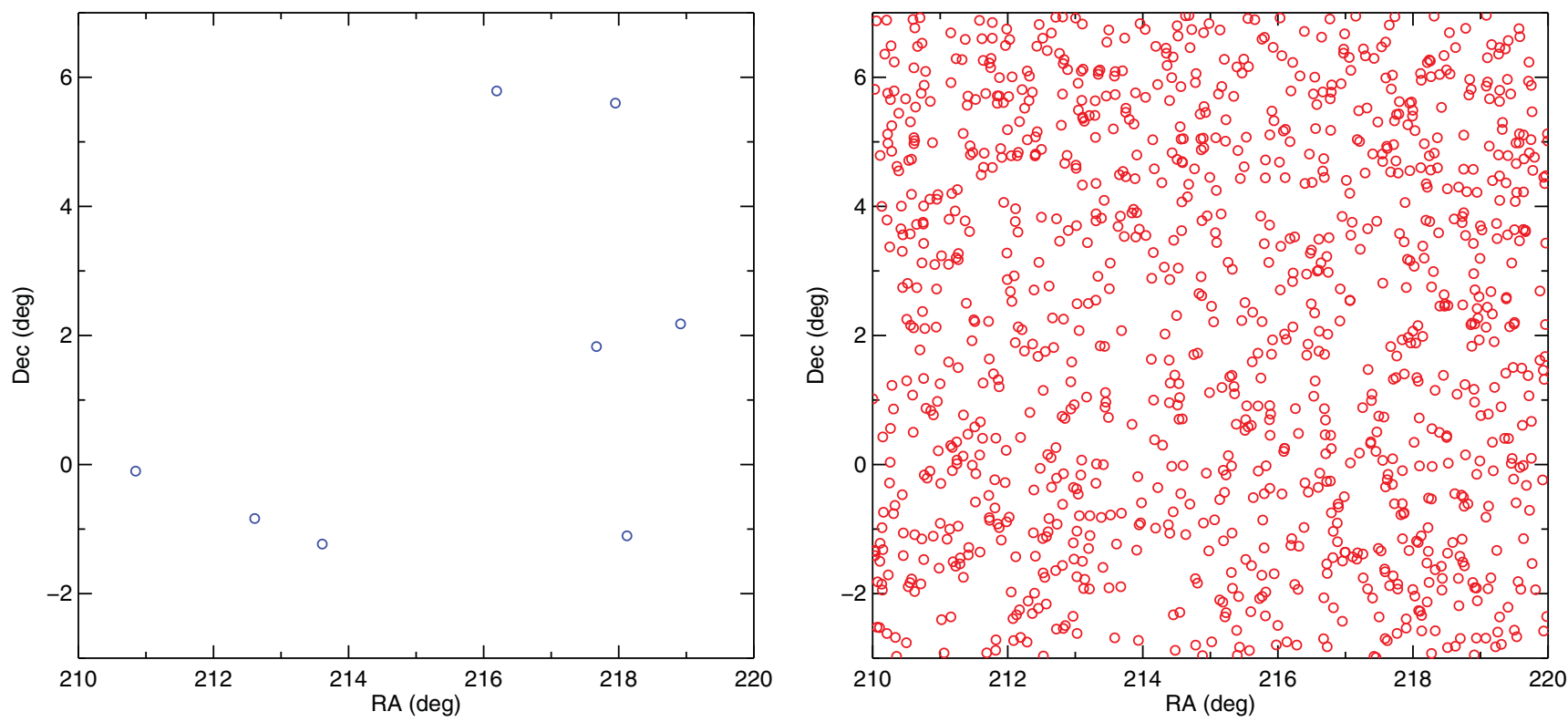

Figure 17. Illustration of the increased density of quasar targets for spectroscopic follow-up made accessible by 30 metre class telescopes. The left-hand panel shows the quasars in a $100 \mathrm{deg}^{2}$ patch of the sky surveyed by the Baryon Oscillation Spectroscopic Survey (BOSS) out to $m_{\mathrm{r}}<18$. These represent targets for which high-quality data can be obtained in $\sim 1 \mathrm{~h}$ of observing using high-resolution spectrographs on current 8 -m telescopes. For contrast, the right-hand panel shows the quasars—in the same patch of sky - that may be readily observed using a high-resolution spectrograph on a 30-m telescope $\left(m_{r}<21\right)$.

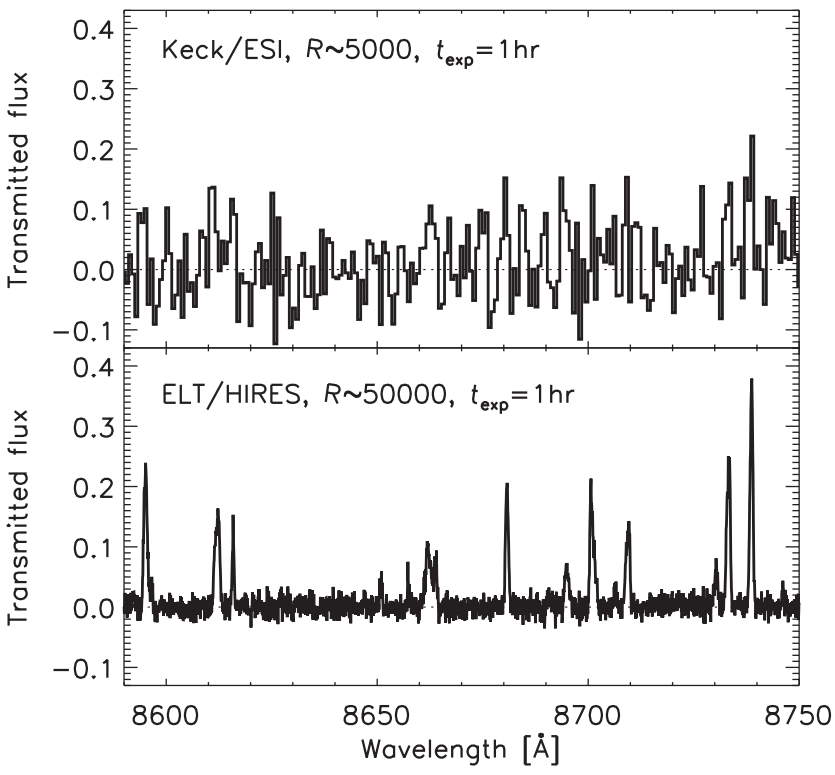

Figure 18. An example of the sensitivity gains enabled by future $30-40$ metre class telescopes. The top panel shows a simulated region of Ly $\alpha$ forest at $z \simeq 6.1$ in the spectrum of a moderate luminosity quasar with magnitude $z_{\mathrm{AB}}=20.5$ as it would be observed in roughly a one-hour exposure with the 10 metre Keck/Echellete Spectrograph and Imager (ESI). The bottom panels shows the same spectrum obtained with a high-resolution spectrograph on a 39 metre telescope. Note the narrow transmission peaks from ionised regions which are now clearly detected and resolved.

In addition to the higher density of observable sightlines, it will be practical to obtain higher resolution and signal-tonoise ratio spectra in relatively short exposure times. This is illustrated in Figure 18, which shows mock spectra of the
Ly $\alpha$ forest at $z=6.1$, comparing what is presently achievable (at a spectral resolution of $R \sim 5000$, e.g. White et al. 2003; Fan et al. 2006) with what will be accessible to a highresolution $(R \sim 50000)$ spectrograph on a 30 metre class telescope. This improved spectroscopy will make it easier to discern whether 'fully absorbed' regions in the forest are truly saturated, facilitate the detection of weak metal absorption lines, and will help resolve Ly $\alpha$ line-widths, facilitating measurements of the temperature of the IGM at $z \geq 5$ (e.g. Becker et al. 2011a; Trac et al. 2008; Furlanetto \& Oh 2009; Lidz \& Malloy 2014), including temperature measurements in the quasar near-zones at $z \geq 6$ (Bolton et al. 2012; Raskutti et al. 2012).

In conjunction with improved quasar absorption line studies, we expect an enhanced interplay with other reionisation probes in the near-future. The James Webb Space Telescope (JWST) is scheduled to launch in 2018, and will allow detailed studies of early galaxy populations in the infrared, while the Atacama Large Millimeter Array (ALMA) may detect the same galaxies at sub-millimeter wavelengths through dust emission, and molecular and atomic fine-structure emission lines (e.g. Maiolino et al. 2015; Capak et al. 2015; Willott et al. 2015; Watson et al. 2015). The Subaru Hyper Suprime-Cam will map-out the positions of sizable populations of LAEs out to $z=7.3$ over wide regions of the sky, enabling improved measurements of the abundance and clustering of these galaxies; these observations will in turn inform our understanding of the ionisation state of the surrounding IGM. Finally, redshifted $21-\mathrm{cm}$ surveys will provide a direct probe of the neutral hydrogen in the IGM during the EoR, and potentially earlier phases of cosmic structure formation 
(Furlanetto et al. 2006a; Paciga et al. 2013; Yatawatta et al. 2013; Parsons et al. 2014; Dillon et al. 2014). Insights gained from quasar absorption lines can help plan and optimise the observing strategy for many of the other future surveys. In addition, absorption line studies will continue to play an important role in piecing together a consistent picture of the EoR in conjunction with the full suite of upcoming observations. For example, quasar near-zone measurements, LAE abundance and clustering observations, GRB optical afterglow spectra, and redshifted $21-\mathrm{cm}$ surveys may all provide independent measurements of the volume-averaged neutral fraction over overlapping redshift ranges. Metal absorption lines, combined with direct galaxy observations with JWST and ALMA will further help to reveal the nature of the galaxies responsible for reionisation. In summary, the prospects for greatly expanding our understanding of the reionisation epoch are excellent over the next several years.

\section{ACKNOWLEDGEMENTS}

We thank Kristian Finlator, Emma Ryan-Weber, Rob Simcoe, and the anonymous referee for helpful comments. JSB acknowledges the support of a Royal Society University Research Fellowship. AL acknowledges support from NASA grant NNX12AC97G and from the NSF through grant AST-1109156.

\section{REFERENCES}

Agafonova, I. I., Levshakov, S. A., Reimers, D., Fechner, C., Tytler, D., Simcoe, R. A., \& Songaila, A. 2007, A\&A, 461, 893

Aghanim, N., Desert, F. X., Puget, J. L., \& Gispert, R. 1996, A\&A, 311,1

Alvarez, M. A., \& Abel, T. 2012, ApJ, 747, 126

Alvarez, M. A., Finlator, K., \& Trenti, M. 2012, ApJ, 759, L38

Bagla, J. S., Kulkarni, G., \& Padmanabhan, T. 2009, MNRAS, 397, 971

Bahcall, J. N., \& Peebles, P. J. E. 1969, ApJ, 156, L7

Bajtlik, S., Duncan, R. C., \& Ostriker, J. P. 1988, ApJ, 327, 570

Bañados, E., et al. 2014, AJ, 148, 14

Bauer, A., Springel, V., Vogelsberger, M., Genel, S., Torrey, P., Sijacki, D., Nelson, D., \& Hernquist, L. 2015, MNRAS, 453, 3593

Becker, G. D., \& Bolton, J. S. 2013, MNRAS, 436, 1023

Becker, G. D., Bolton, J. S., Haehnelt, M. G., \& Sargent, W. L. W. 2011a, MNRAS, 410, 1096

Becker, G. D., Bolton, J. S., Madau, P., Pettini, M., Ryan-Weber, E. V., \& Venemans, B. P. 2015, MNRAS, 447, 3402

Becker, G. D., Hewett, P. C., Worseck, G., \& Prochaska, J. X. 2013, MNRAS, 430, 2067

Becker, G. D., Rauch, M., \& Sargent, W. L. W. 2009, ApJ, 698, 1010

Becker, G. D., Sargent, W. L. W., Rauch, M., \& Calverley, A. P. 2011b, ApJ, 735, 93

Becker, G. D., Sargent, W. L. W., Rauch, M., \& Carswell, R. F. 2012, ApJ, 744, 91

Becker, G. D., Sargent, W. L. W., Rauch, M., \& Simcoe, R. A. 2006, ApJ, 640, 69

Becker, R. H., et al. 2001, AJ, 122, 2850

Behroozi, P. S., Wechsler, R. H., \& Conroy, C. 2013, ApJ, 770, 57
Bennett, C. L., et al. 2013, ApJS, 208, 20

Boera, E., Murphy, M. T., Becker, G. D., \& Bolton, J. S. 2014, MNRAS, 441, 1916

Boksenberg, A., \& Sargent, W. L. W. 2015, ApJS, 218, 7

Bolton, J., Meiksin, A., \& White, M. 2004, MNRAS, 348, L43

Bolton, J. S., \& Becker, G. D. 2009, MNRAS, 398, L26

Bolton, J. S., Becker, G. D., Haehnelt, M. G., \& Viel, M. 2014, MNRAS, 438, 2499

Bolton, J. S., Becker, G. D., Raskutti, S., Wyithe, J. S. B., Haehnelt, M. G., \& Sargent, W. L. W. 2012, MNRAS, 419, 2880

Bolton, J. S., Becker, G. D., Wyithe, J. S. B., Haehnelt, M. G., \& Sargent, W. L. W. 2010, MNRAS, 406, 612

Bolton, J. S., \& Haehnelt, M. G. 2007a, MNRAS, 374, 493

Bolton, J. S., \& Haehnelt, M. G. 2007b, MNRAS, 381, L35

Bolton, J. S., \& Haehnelt, M. G. 2007c, MNRAS, 382, 325

Bolton, J. S., \& Haehnelt, M. G. 2013, MNRAS, 429, 1695

Bolton, J. S., Haehnelt, M. G., Viel, M., \& Springel, V. 2005, MNRAS, 357, 1178

Bolton, J. S., Haehnelt, M. G., Warren, S. J., Hewett, P. C., Mortlock, D. J., Venemans, B. P., McMahon, R. G., \& Simpson, C. 2011, MNRAS, 416, L70

Bordoloi, R., et al. 2014, ApJ, 796, 136

Bosman, S. E. I., \& Becker, G. D. 2015, MNRAS, 452, 1105

Boutsia, K., et al. 2011, MNRAS, 452, 1105

Bouwens, R. J., et al. 2014, ApJ, 793, 115

Bouwens, R. J., et al. 2015, ApJ, 803, 34

Bowler, R. A. A., et al. 2014, MNRAS, 440, 2810

Calverley, A. P., Becker, G. D., Haehnelt, M. G., \& Bolton, J. S. 2011, MNRAS, 412, 2543

Capak, P. L., et al. 2015, Natur, 522, 455

Carilli, C. L., et al. 2010, ApJ, 714, 834

Carswell, R. F., Whelan, J. A. J., Smith, M. G., Boksenberg, A., \& Tytler, D. 1982, MNRAS, 198, 91

Castro-Tirado, A. J., et al. 2013, arXiv:1312.5631

Cayrel, R., et al. 2004, A\&A, 416, 1117

Cen, R., \& Chisari, N. E. 2011, ApJ, 731, 11

Chardin, J., Haehnelt, M. G., Aubert, D., \& Puchwein, E. 2015, MNRAS, 453, 2943

Chieffi, A., \& Limongi, M. 2004, ApJ, 608, 405

Chornock, R., Berger, E., Fox, D. B., Lunnan, R., Drout, M. R., fai Fong, W., Laskar, T., \& Roth, K. C. 2013, ApJ, 774, 26

Choudhury, T. R., Haehnelt, M. G., \& Regan, J. 2009, MNRAS, 394, 960

Choudhury, T. R., Puchwein, E., Haehnelt, M. G., \& Bolton, J. S. 2015, MNRAS, 452, 261

Ciardi, B., Bolton, J. S., Maselli, A., \& Graziani, L. 2012, MNRAS, 423, 558

Compostella, M., Cantalupo, S., \& Porciani, C. 2014, MNRAS, 445, 4186

Cooke, R., Pettini, M., Steidel, C. C., Rudie, G. C., \& Nissen, P. E. 2011, MNRAS, 417, 1534

Cooksey, K. L., Kao, M. M., Simcoe, R. A., O’Meara, J. M., \& Prochaska, J. X. 2013, ApJ, 763, 37

Cooksey, K. L., Thom, C., Prochaska, J. X., \& Chen, H.-W. 2010, ApJ, 708, 868

Cowie, L. L., Barger, A. J., \& Trouille, L. 2009, ApJ, 692, 1476

Crighton, N. H. M., et al. 2015, MNRAS, 452, 217

Croom, S. M., et al. 2009, MNRAS, 399, 1755

Dall'Aglio, A., Wisotzki, L., \& Worseck, G. 2008, A\&A, 491, 465

Danforth, C. W., \& Shull, J. M. 2008, ApJ, 679, 194

Danforth, C. W., et al. 2014, ApJ, arXiv:1402:2655 
Davé, R., \& Oppenheimer, B. D. 2007, MNRAS, 374, 427

Dessauges-Zavadsky, M., Péroux, C., Kim, T.-S., D’Odorico, S., \& McMahon, R. G. 2003, MNRAS, 345, 447

Díaz, C. G., Koyama, Y., Ryan-Weber, E. V., Cooke, J., Ouchi, M., Shimasaku, K., \& Nakata, F. 2014, MNRAS, 442, 946

Díaz, C. G., Ryan-Weber, E. V., Cooke, J., Koyama, Y., \& Ouchi, M. 2015, MNRAS, 448, 1240

Díaz, C. G., Ryan-Weber, E. V., Cooke, J., Pettini, M., \& Madau, P. 2011, MNRAS, 418, 820

Dijkstra, M. 2014, PASA, 31, 40

Dijkstra, M., Haiman, Z., \& Loeb, A. 2004a, ApJ, 613, 646

Dijkstra, M., Lidz, A., \& Hui, L. 2004b, ApJ, 605, 7

Dijkstra, M., Wyithe, S., Haiman, Z., Mesinger, A., \& Pentericci, L. 2014, MNRAS, 440, 3309

Dillon, J. S., et al. 2014, PRD, 89, 023002

Djorgovski, S. G., Castro, S., Stern, D., \& Mahabal, A. A. 2001, ApJ, 560, L5

D'Odorico, S., et al. 2006, Proc. SPIE, 6269, 626933

D’Odorico, V., Calura, F., Cristiani, S., \& Viel, M. 2010, MNRAS, 401,2715

D'Odorico, V., et al. 2013, MNRAS, 435, 1198

Duncan, K., \& Conselice, C. J. 2015, MNRAS, 451, 2030

Dunlop, J. S., et al. 2013, MNRAS, 432, 3520

Eldridge, J. J., \& Stanway, E. R. 2012, MNRAS, 419, 479

Emberson, J. D., Thomas, R. M., \& Alvarez, M. A. 2013, ApJ, 763, 146

Fabbian, D., Nissen, P. E., Asplund, M., Pettini, M., \& Akerman, C. 2009, A\&A, 500, 1143

Fan, X., et al. 2006, AJ, 132, 117

Faucher-Giguère, C.-A., Lidz, A., Hernquist, L., \& Zaldarriaga, M. 2008b, ApJ, 688, 85

Faucher-Giguère, C.-A., Lidz, A., Zaldarriaga, M., \& Hernquist, L. 2009, ApJ, 703, 1416

Faucher-Giguère, C.-A., Prochaska, J. X., Lidz, A., Hernquist, L., \& Zaldarriaga, M. 2008a, ApJ, 681, 831

Fechner, C. 2011, A\&A, 532, A62

Ferrara, A., \& Loeb, A. 2013, MNRAS, 431, 2826

Finkelstein, S. L., et al. 2012, ApJ, 756, 164

Finkelstein, S. L., et al. 2015, ApJ, 810, 71

Finlator, K., Oh, S. P., Özel, F., \& Davé, R. 2012, MNRAS, 427, 2464

Finlator, K., Muñoz, J. A., Oppenheimer, B. D., Oh, S. P., Özel, F., \& Davé, R. 2013, MNRAS, 436, 1818

Finlator, K., Thompson, R., Huang, S., Davé, R., Zackrisson, E., \& Oppenheimer, B. D. 2015, MNRAS, 447, 2526

Fontanot, F., Cristiani, S., Pfrommer, C., Cupani, G., \& Vanzella, E. 2014, MNRAS, 438, 2097

Friedrich, M. M., Mellema, G., Iliev, I. T., \& Shapiro, P. R. 2012, MNRAS, 421, 2232

Fumagalli, M., O’Meara, J. M., Prochaska, J. X., \& Worseck, G. 2013, ApJ, 775, 78

Furlanetto, S. R., \& Loeb, A. 2003, ApJ, 588, 18

Furlanetto, S. R., \& Mesinger, A. 2009, MNRAS, 394, 1667

Furlanetto, S. R., \& Oh, S. P. 2005, MNRAS, 363, 1031

Furlanetto, S. R., \& Oh, S. P. 2008, ApJ, 681, 1

Furlanetto, S. R., \& Oh, S. P. 2009, ApJ, 701, 94

Furlanetto, S. R., Oh, S. P., \& Briggs, F. H. 2006a, PhR, 433, 181

Furlanetto, S. R., Zaldarriaga, M., \& Hernquist, L. 2004, ApJ, 613, 1

Furlanetto, S. R., Zaldarriaga, M., \& Hernquist, L. 2006b, MNRAS, 365,1012
Gallerani, S., Choudhury, T. R., \& Ferrara, A. 2006, MNRAS, 370, 1401

Gallerani, S., Ferrara, A., Fan, X., \& Choudhury, T. R. 2008, MNRAS, 386, 359

Garzilli, A., Bolton, J. S., Kim, T.-S., Leach, S., \& Viel, M. 2012, MNRAS, 424, 1723

George, E. M., et al. 2015, ApJ, 799, 177

Giallongo, E., Cristiani, S., D’Odorico, S., Fontana, A., \& Savaglio S. 1996, ApJ, 466, 46

Giallongo, E., Menci, N., Fiore, F., Castellano, M., Fontana, A., Grazian, A., \& Pentericci, L. 2012, ApJ, 755, 124

Giallongo, E., et al. 2015, A\&A, 578, A83

Glikman, E., Djorgovski, S. G., Stern, D., Dey, A., Jannuzi, B. T., \& Lee, K.-S. 2011, ApJ, 728, L26

Gnedin, N. Y. 2000, ApJ, 535, 530

Gnedin, N. Y. 2014, ApJ, 793, 29

Gnedin, N. Y., \& Fan, X. 2006, ApJ, 648, 1

González, V., Labbé, I., Bouwens, R. J., Illingworth, G., Franx, M., \& Kriek, M. 2011, ApJ, 735, L34

Gruzinov, A., \& Hu, W. 1998, ApJ, 508, 435

Guimarães, R., Petitjean, P., de Carvalho, R. R., Djorgovski, S. G., Noterdaeme, P., Castro, S., Poppe, P. C. D. R., \& Aghaee, A. 2009, A\&A, 508, 133

Guimarães, R., Petitjean, P., Rollinde, E., de Carvalho, R. R., Djorgovski, S. G., Srianand, R., Aghaee, A., \& Castro, S. 2007, MNRAS, 377, 657

Gunn, J. E., \& Peterson, B. A. 1965, ApJ, 142, 1633

Haardt, F., \& Madau, P. 1996, ApJ, 461, 20

Haardt, F., \& Madau, P. 2012, ApJ, 746, 125

Haardt, F., \& Salvaterra, R. 2015, A\&A, 575, L16

Hartoog, O. E., et al. 2015, A\&A, 580, A139

Hopkins, P. F., Richards, G. T., \& Hernquist, L. 2007, ApJ, 654, 731

Hu, W., \& White, M. 1997, New A, 2, 323

Hui, L., \& Gnedin, N. Y. 1997, MNRAS, 292, 27

Hui, L., \& Haiman, Z. 2003, ApJ, 596, 9

Ishigaki, M., Kawamata, R., Ouchi, M., Oguri, M., Shimasaku, K., \& Ono, Y. 2015, ApJ, 799, 12

Jeeson-Daniel, A., Ciardi, B., \& Graziani, L. 2014, MNRAS, 443, 2722

Kaiser, N., \& Peacock, J. A. 1991, ApJ, 379, 482

Kaurov, A. A., \& Gnedin, N. Y. 2014, ApJ, 787, 146

Keating, L. C., Haehnelt, M. G., Becker, G. D., \& Bolton, J. S. 2014, MNRAS, 438, 1820

Keating, L. C., Haehnelt, M. G., Cantalupo, S., \& Puchwein, E. 2015, MNRAS, 454, 681

Kennicutt, R. C., 1998, ARAA, 36, 189

Kim, T.-S., Carswell, R. F., Cristiani, S., D'Odorico, S., \& Giallongo, E. 2002, MNRAS, 335, 555

Kim, T.-S., Hu, E. M., Cowie, L. L., \& Songaila, A. 1997, AJ, 114, 1

Kim, T.-S., Partl, A. M., Carswell, R. F., \& Müller, V. 2013, A\&A, 552, A77

Kirkman, D., et al. 2005, MNRAS, 360, 1373

Konno, A., et al. 2014, ApJ, 797, 16

Kramer, R. H., \& Haiman, Z. 2009, MNRAS, 400, 1493

Kuhlen, M., \& Faucher-Giguère, C.-A. 2012, MNRAS, 423, 862

Kulkarni, G., Hennawi, J. F., Rollinde, E., \& Vangioni, E. 2014, ApJ, 787, 64

Leitherer, C., et al. 1999, ApJS, 123, 3 
Lidz, A., Faucher-Giguère, C.-A., Dall'Aglio, A., McQuinn, M., Fechner, C., Zaldarriaga, M., Hernquist, L., \& Dutta, S. 2010, ApJ, 718, 199

Lidz, A., \& Malloy, M. 2014, ApJ, 788, 175

Lidz, A., McQuinn, M., Zaldarriaga, M., Hernquist, L., \& Dutta, S. 2007, ApJ, 670, 39

Lidz, A., Oh, S. P., \& Furlanetto, S. R. 2006, ApJ, 639, L47

Loeb, A., \& Eisenstein, D. J. 1995, ApJ, 448, 17

Loeb, A., \& Furlanetto, S. R. 2013, The First Galaxies in the Universe (Princeton, NJ: Princeton Univ. Press)

Madau, P., Haardt, F., \& Rees, M. J. 1999, ApJ, 514, 648

Madau, P., Meiksin, A., \& Rees, M. J. 1997, ApJ, 475, 429

Maiolino, R., et al. 2015, MNRAS, 452, 54

Malloy, M., \& Lidz, A. 2015, ApJ, 799, 179

Maselli, A., Ferrara, A., \& Gallerani, S. 2009, MNRAS, 395, 1925

Maselli, A., Gallerani, S., Ferrara, A., \& Choudhury, T. R. 2007, MNRAS, 376, L34

Masters, D., et al. 2012, ApJ, 755, 169

Matejek, M. S., \& Simcoe, R. A. 2012, ApJ, 761, 112

Matejek, M. S., Simcoe, R. A., Cooksey, K. L., \& Seyffert, E. N. 2013, ApJ, 764, 9

McDonald, P., \& Miralda-Escudé, J. 2001, ApJ, 549, L11

McDonald, P., Miralda-Escudé, J., Rauch, M., Sargent, W. L. W., Barlow, T. A., \& Cen, R. 2001, ApJ, 562, 52

McGreer, I. D., Mesinger, A., \& D'Odorico, V. 2015, MNRAS, 447, 499

McGreer, I. D., Mesinger, A., \& Fan, X. 2011, MNRAS, 415, 3237

McLeod, D. J., McLure, R. J., Dunlop, J. S., Robertson, B. E., Ellis, R. S., \& Targett, T. A. 2015, MNRAS, 450, 3032

McLure, R. J., et al. 2013, MNRAS, 432, 2696

McQuinn, M. 2012, MNRAS, 426, 1349

McQuinn, M., Furlanetto, S. R., Hernquist, L., Zahn, O., \& Zaldarriaga, M. 2005, ApJ, 630, 643

McQuinn, M., Hernquist, L., Zaldarriaga, M., \& Dutta, S. 2007b, MNRAS, 381, 75

McQuinn, M., Lidz, A., Zahn, O., Dutta, S., Hernquist, L., \& Zaldarriaga, M. 2007a, MNRAS, 377, 1043

McQuinn, M., Lidz, A., Zaldarriaga, M., Hernquist, L., \& Dutta, S. 2008, MNRAS, 388, 1101

McQuinn, M., Lidz, A., Zaldarriaga, M., Hernquist, L., Hopkins, P. F., Dutta, S., \& Faucher-Giguère, C.-A. 2009, ApJ, 694, 842

McQuinn, M., Oh, S. P., \& Faucher-Giguère, C.-A. 2011, ApJ, 743, 82

McQuinn, M., \& Upton Sanderbeck, P. 2015, arXiv: 1505.07875

Meiksin, A. 2005, MNRAS, 356, 596

Meiksin, A. A. 2009, RvMP, 81, 1405

Meiksin, A., \& White, M. 2003, MNRAS, 342, 1205

Meiksin, A., \& White, M. 2004, MNRAS, 350, 1107

Ménard, B., Wild, V., Nestor, D., Quider, A., Zibetti, S., Rao S., \& Turnshek, D. 2011, MNRAS, 417, 801

Mesinger, A. 2010, MNRAS, 407, 1328

Mesinger, A., \& Furlanetto, S. R. 2008a, MNRAS, 385, 1348

Mesinger, A., \& Furlanetto, S. R. 2008b, MNRAS, 386, 1990

Mesinger, A., \& Furlanetto, S. 2009, MNRAS, 400, 1461

Mesinger, A., \& Haiman, Z. 2004, ApJ, 611, L69

Mesinger, A., \& Haiman, Z. 2007, ApJ, 660, 923

Mesinger, A., Aykutalp, A., Vanzella, E., Pentericci, L., Ferrara, A., \& Dijkstra, M. 2015, MNRAS, 446, 566
Mesinger, A., McQuinn, M., \& Spergel, D. N. 2012, MNRAS, 422, 1403

Miralda-Escudé, J. 1998, ApJ, 501, 15

Miralda-Escudé, J. 2003, ApJ, 597, 66

Miralda-Escudé, J., Haehnelt, M., \& Rees, M. J. 2000, ApJ, 530, 1

Miralda-Escudé, J., \& Rees, M. J. 1994, MNRAS, 266, 343

Mitra, S., Ferrara, A., \& Choudhury, T. R. 2013, MNRAS, 428, L1

Mortlock, D. J., et al. 2011, Natur, 474, 616

Mostardi, R. E., Shapley, A. E., Nestor, D. B., Steidel, C. C., Reddy, N. A., \& Trainor, R. F. 2013, ApJ, 779, 65

Muñoz, J. A., Oh, S. P., Davies, F. B., \& Furlanetto, S. R. 2016, MNRAS, 455, 1385

Murdoch, H. S., Hunstead, R. W., Pettini, M., \& Blades, J. C. 1986, ApJ, 309, 19

Murray, S. G., Power, C., \& Robotham, A. S. G. 2013, A\&C, 3, 23

Nestor, D. B., Shapley, A. E., Kornei, K. A., Steidel, C. C., \& Siana, B. 2013, ApJ, 765, 47

Nestor, D. B., Turnshek, D. A., \& Rao, S. M. 2005, ApJ, 628, 637

Noterdaeme, P., Petitjean, P., Ledoux, C., \& Srianand, R. 2009, A\&A, 505, 1087

Oesch, P. A., et al. 2013, ApJ, 773, 75

Oh, S. P. 2002, MNRAS, 336, 1021

Oh, S. P., \& Furlanetto, S. R. 2005, ApJ, 620, L9

O’Meara, J. M., Prochaska, J. X., Burles, S., Prochter, G., Bernstein, R. A., \& Burgess, K. M. 2007, ApJ, 656, 666

O'Meara, J. M., Prochaska, J. X., Worseck, G., Chen, H.-W., \& Madau, P. 2013, ApJ, 765, 137

Oppenheimer, B. D., \& Davé, R. 2006, MNRAS, 373, 1265

Oppenheimer, B. D., \& Davé, R. 2008, MNRAS, 387, 577

Oppenheimer, B. D., Davé, R., \& Finlator, K. 2009, MNRAS, 396, 729

Osterbrock, D. E., \& Ferland, G. J. 2006, Astrophysics of Gaseous Nebulae and Active Galactic Nuclei (2nd edn.; Sausalito: University Science Books), 20

Ota, K., et al. 2008, ApJ, 677, 12

Ouchi, M., et al. 2010, ApJ, 723, 869

Paciga, G., et al. 2013, MNRAS, 433, 639

Padmanabhan, H., Choudhury, T. R., \& Srianand, R. 2014, MNRAS, 443, 3761

Pallottini, A., Ferrara, A., Gallerani, S., Salvadori, S., \& D’Odorico, V. 2014, MNRAS, 440, 2498

Paresce, F., McKee, C. F., \& Bowyer, S. 1980, ApJ, 240, 387

Pâris, I., et al. 2011, A\&A, 530, A50

Parsons, A. R., et al. 2014, ApJ, 788, 106

Partl, A. M., Müller, V., Yepes, G., \& Gottlöber, S. 2011, MNRAS, 415,3851

Paschos, P., \& Norman, M. L. 2005, ApJ, 631, 59

Pawlik, A. H., Schaye, J., \& van Scherpenzeel, E. 2009, MNRAS, 394, 1812

Peeples, M. S., Werk, J. K., Tumlinson, J., Oppenheimer, B. D., Prochaska, J. X., Katz, N., \& Weinberg, D. H. 2014, ApJ, 786, 54

Pentericci, L., et al. 2014, ApJ, 793, 113

Péroux, C., Dessauges-Zavadsky, M., D’Odorico, S., Kim, T. S., \& McMahon, R. G. 2005, MNRAS, 363, 479

Péroux, C., Dessauges-Zavadsky, M., D’Odorico, S., Kim, T.-S., \& McMahon, R. G. 2007, MNRAS, 382, 177

Petitjean, P., Webb, J. K., Rauch, M., Carswell, R. F., \& Lanzetta, K. 1993, MNRAS, 262, 499 
Pettini, M. 1999, Chemical Evolution from Zero to High Redshift, in Proceedings of the ESO Workshop, ed. Jeremy R. Walsh and Michael R. Rosa (Berlin: Springer-Verlag)

Pettini, M., Madau, P., Bolte, M., Prochaska, J. X., Ellison, S. L., \& Fan, X. 2003, ApJ, 594, 695

Planck Collaboration XVI. 2014, A\&A, 571, A16

Planck Collaboration XIII. 2015, arXiv:1502.01589

Pritchard, J. R., Loeb, A., \& Wyithe, J. S. B. 2010, MNRAS, 408, 57

Prochaska, J. X., Madau, P., O’Meara, J. M., \& Fumagalli, M. 2014 , MNRAS, 438, 476

Prochaska, J. X., O’Meara, J. M., \& Worseck, G. 2010, ApJ, 718, 392

Prochaska, J. X., \& Wolfe, A. M. 2009, ApJ, 696, 1543

Prochaska, J. X., Worseck, G., \& O’Meara, J. M. 2009, ApJ, 705, L113

Prochter, G. E., Prochaska, J. X., \& Burles, S. M. 2006, ApJ, 639, 766

Rafelski, M., Neeleman, M., Fumagalli, M., Wolfe, A. M., \& Prochaska, J. X. 2014, ApJ, 782, L29

Raskutti, S., Bolton, J. S., Wyithe, J. S. B., \& Becker, G. D. 2012, MNRAS, 421, 1969

Rauch, M. 1998, ARAA, 36, 267

Rauch, M., et al. 1997, ApJ, 489, 7

Reed, S. L., et al. 2015, MNRAS, 454, 3952

Richards, G. T., et al. 2006, AJ, 131, 2766

Ricotti, M., Gnedin, N. Y., \& Shull, J. M. 2000, ApJ, 534, 41

Robertson, B. E., Ellis, R. S., Furlanetto, S. R., \& Dunlop, J. S. 2015, ApJ, 802, L19

Robertson, B. E., et al. 2013, ApJ, 768, 71

Rollinde, E., Srianand, R., Theuns, T., Petitjean, P., \& Chand, H. 2005, MNRAS, 361, 1015

Rollinde, E., Theuns, T., Schaye, J., Pâris, I., \& Petitjean, P. 2013, MNRAS, 428, 540

Rorai, A., Hennawi, J. F., \& White, M. 2013, ApJ, 775, 81

Rudie, G. C., Steidel, C. C., \& Pettini, M. 2012, ApJ, 757, L30

Rudie, G. C., Steidel, C. C., Shapley, A. E., \& Pettini, M. 2013, ApJ, 769, 146

Ryan-Weber, E. V., Pettini, M., \& Madau, P. 2006, MNRAS, 371, L78

Ryan-Weber, E. V., Pettini, M., Madau, P., \& Zych, B. J. 2009, MNRAS, 395, 1476

Schaye, J., Aguirre, A., Kim, T.-S., Theuns, T., Rauch, M., \& Sargent, W. L. W. 2003, ApJ, 596, 768

Schaye, J., Theuns, T., Rauch, M., Efstathiou, G., \& Sargent, W. L. W. 2000, MNRAS, 318, 817

Schechter, P. 1976, ApJ, 203, 297

Schenker, M. A., Ellis, R. S., Konidaris, N. P., \& Stark, D. P. 2014, ApJ, 79520

Schirber, M., \& Bullock, J. S. 2003, ApJ, 584, 110

Schmidt, M. 1965, ApJ, 141, 1295

Schroeder, J., Mesinger, A., \& Haiman, Z. 2013, MNRAS, 428, 3058

Scott, J., Bechtold, J., Dobrzycki, A., \& Kulkarni, V. P. 2000, ApJS, 130, 67

Seyffert, E. N., Cooksey, K. L., Simcoe, R. A., O'Meara, J. M., Kao, M. M., \& Prochaska, J. X. 2013, ApJ, 779, 161

Sheth, R. K., \& Tormen, G. 2002, MNRAS, 329, 61

Shull, J. M., Danforth, C. W., \& Tilton, E. M. 2014, ApJ, 796, 49

PASA, 32, e045 (2015)

doi:10.1017/pasa.2015.45
Shull, J. M., France, K., Danforth, C. W., Smith, B., \& Tumlinson, J. 2010, ApJ, 722, 1312

Shull, J. M., Harness, A., Trenti, M., \& Smith, B. D. 2012, ApJ, 747,100

Simcoe, R. A. 2006, ApJ, 653, 977

Simcoe, R. A., Sargent, W. L. W., Rauch, M., \& Becker, G. 2006, ApJ, 637, 648

Simcoe, R. A., Sullivan, P. W., Cooksey, K. L., Kao, M. M., Matejek, M. S., \& Burgasser, A. J. 2012, Natur, 492, 79

Simcoe, R. A., et al. 2011, ApJ, 743, 21

Simcoe, R. A., et al. 2013, PASP, 125, 270

So, G. C., Norman, M. L., Reynolds, D. R., \& Wise, J. H. 2014, ApJ, 789, 149

Sobacchi, E., \& Mesinger, A. 2014, MNRAS, 440, 1662

Songaila, A. 2004, AJ, 127, 2598

Songaila, A., \& Cowie, L. L. 2002, AJ, 123, 2183

Songaila, A., \& Cowie, L. L. 2010, ApJ, 721, 1448

Stark, D. P., Schenker, M. A., Ellis, R., Robertson, B., McLure, R., \& Dunlop, J. 2013, ApJ, 763, 129

Steidel, C. C., Erb, D. K., Shapley, A. E., Pettini, M., Reddy, N., Bogosavljević, M., Rudie, G. C., \& Rakic, O. 2010, ApJ, 717, 289

Stevans, M. L., Shull, J. M., Danforth, C. W., \& Tilton, E. M. 2014, ApJ, 794, 75

Storrie-Lombardi, L. J., McMahon, R. G., Irwin, M. J., \& Hazard, C. 1994, ApJ, 427, L13

Taylor, J., \& Lidz, A. 2014, MNRAS, 437, 2542

Telfer, R. C., Zheng, W., Kriss, G. A., \& Davidsen, A. F. 2002, ApJ, 565,773

Theuns, T., Schaye, J., Zaroubi, S., Kim, T.-S., Tzanavaris, P., \& Carswell, B. 2002, ApJ, 567, L103

Tilton, E. M., Danforth, C. W., Shull, J. M., \& Ross, T. L. 2012, ApJ, 759, 112

Totani, T., Kawai, N., Kosugi, G., Aoki, K., Yamada, T., Iye, M., Ohta, K., \& Hattori, T. 2006, PASJ, 58, 485

Totani, T., et al. 2014, PASJ, 66, 63

Trac, H., Cen, R., \& Loeb, A. 2008, ApJ, 689, L81

Trenti, M., Stiavelli, M., Bouwens, R. J., Oesch, P., Shull, J. M., Illingworth, G. D., Bradley, L. D., \& Carollo, C. M. 2010, ApJ, 714, L202

Tumlinson, J., et al. 2011, Sci, 334, 948

Turner, M. L., Schaye, J., Steidel, C. C., Rudie, G. C., \& Strom, A. L. 2014, MNRAS, 445, 794

Tytler, D. 1987, ApJ, 321, 49

Tytler, D., et al. 2004, ApJ, 617, 1

Ueda, Y., Akiyama, M., Ohta, K., \& Miyaji, T. 2003, ApJ, 598, 886

Vanzella, E., et al. 2010, ApJ, 725, 1011

Venemans, B. P., et al. 2013, ApJ, 779, 24

Venemans, B. P., et al. 2015, ApJ, 801, L11

Verner, D. A., \& Ferland, G. J. 1996, ApJS, 103, 467

Verner, D. A., Ferland, G. J., Korista, K. T., \& Yakovlev, D. G. 1996, ApJ, 465, 487

Watson, D., Christensen, L., Knudsen, K. K., Richard, J., Gallazzi, A., \& Michałowski, M. J. 2015, Natur, 519, 327

Weinberg, D. H., et al. 1999, Evolution of Large Scale Structure: From Recombination to Garching, in Proceedings of the MPAESO Cosmology Conference, ed. Banday, A. J., Sheth, R. K. \& L. N. da Costa (Garching, Germany: European Southern Observatory), 346 
Weymann, R. J., Carswell, R. F., \& Smith, M. G. 1981, ARAA, 19, 41

White, R. L., Becker, R. H., Fan, X., \& Strauss, M. A. 2003, AJ, 126,1

Willott, C. J., Carilli, C. L., Wagg, J., \& Wang, R. 2015, ApJ, 807, 180

Wise, J. H., Demchenko, V. G., Halicek, M. T., Norman, M. L., Turk, M. J., Abel, T., \& Smith, B. D. 2014, MNRAS, 442, 2560

Wolfe, A. M., Gawiser, E., \& Prochaska, J. X. 2005, ARAA, 43, 861

Worseck, G., et al. 2011, ApJ, 733, L24

Worseck, G., et al. 2014, MNRAS, 445, 1745

Wyithe, J. S. B., \& Bolton, J. S. 2011, MNRAS, 412, 1926
Wyithe, J. S. B., Bolton, J. S., \& Haehnelt, M. G. 2008, MNRAS, 383, 691

Wyithe, J. S. B., Hopkins, A. M., Kistler, M. D., Yüksel, H., \& Beacom, J. F. 2010, MNRAS, 401, 2561

Wyithe, J. S. B., \& Loeb, A. 2004, Natur, 427, 815

Wyithe, J. S. B., \& Loeb, A. 2006, ApJ, 646, 696

Wyithe, J. S. B., Loeb, A., \& Carilli, C. 2005, ApJ, 628, 575

Yatawatta, S., et al. 2013, A\&A, 550, A136

Zahn, O., Zaldarriaga, M., Hernquist, L., \& McQuinn, M. 2005, ApJ, 630, 657

Zahn, O., et al. 2012, ApJ, 756, 65

Zaldarriaga, M. 1997, PRD, 55, 1822

Zuo, L. 1992, MNRAS, 258, 45 